University of New Hampshire

University of New Hampshire Scholars' Repository

$2-2013$

\title{
County-Specific Net Migration by Five-Year Age Groups, Hispanic Origin, Race and Sex 2000-2010
}

\author{
Richelle Winkler \\ Michigan Technological University \\ Kenneth M. Johnson \\ University of New Hampshire - Main Campus, ken.johnson@unh.edu \\ Cheng Cheng \\ Princeton University \\ Paul R. Voss \\ Odum Institute for Research in Social Science \\ Katherine J. Curtis \\ University of Wisconsin - Madison
}

Follow this and additional works at: https://scholars.unh.edu/soc_facpub

Part of the Demography, Population, and Ecology Commons, and the Social Statistics Commons

\author{
Recommended Citation \\ Winkler, Richelle; Johnson, Kenneth M.; Cheng, Cheng; Voss, Paul R.; and Curtis, Katherine J., "County- \\ Specific Net Migration by Five-Year Age Groups, Hispanic Origin, Race and Sex 2000-2010" (2013). \\ Sociology Scholarship. 85. \\ https://scholars.unh.edu/soc_facpub/85
}

This Report is brought to you for free and open access by the Sociology at University of New Hampshire Scholars' Repository. It has been accepted for inclusion in Sociology Scholarship by an authorized administrator of University of New Hampshire Scholars' Repository. For more information, please contact Scholarly.Communication@unh.edu. 


\section{Center for Demography and Ecology}

University of Wisconsin-Madison

County-Specific Net Migration by Five-Year Age Groups,

Hispanic Origin, Race and Sex 2000-2010

Richelle L. Winkler

Kenneth M. Johnson

Cheng Cheng

Paul R. Voss

Katherine J. Curtis

CDE Working Paper No. 2013-04

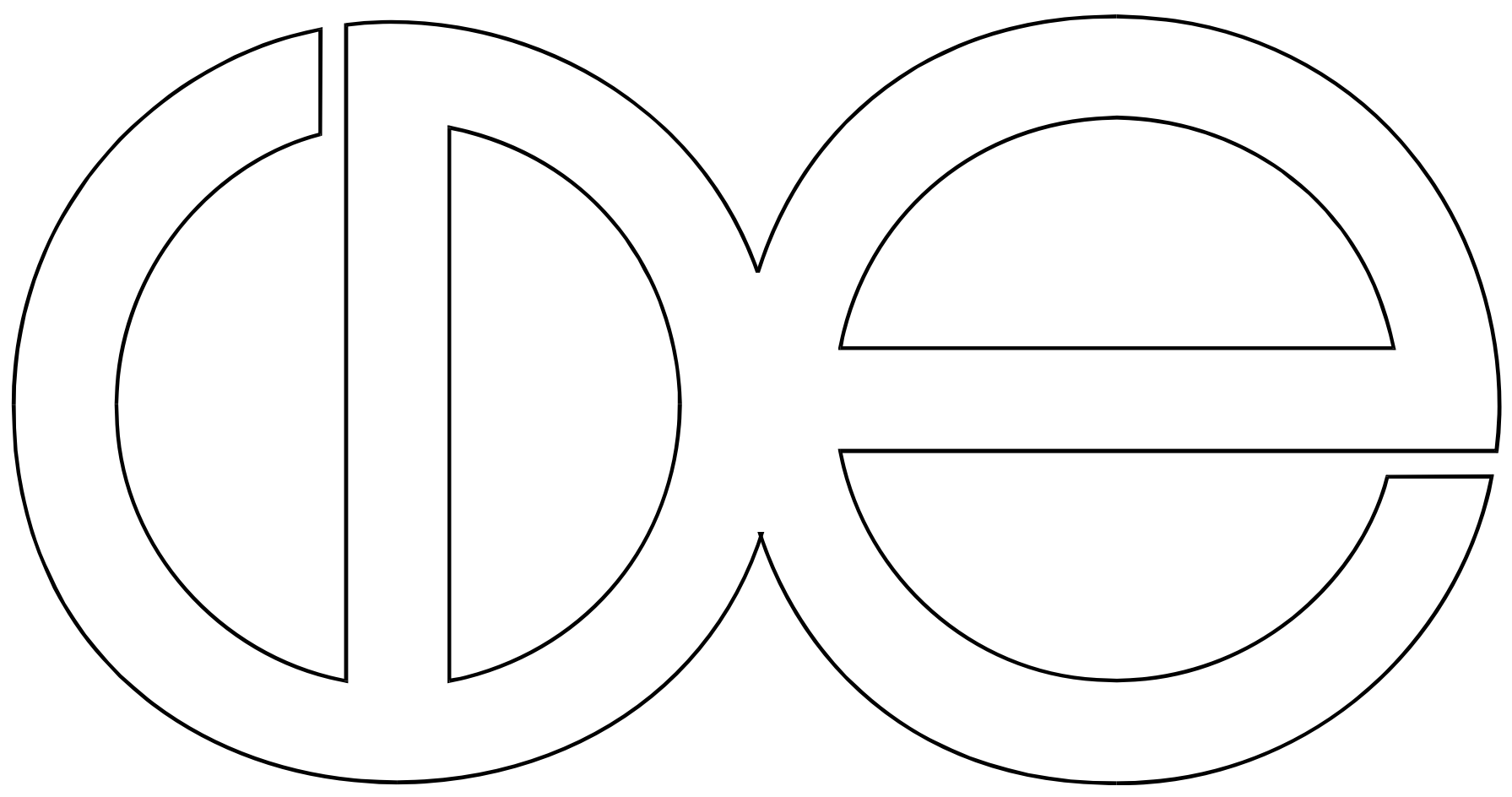




\title{
County-Specific Net Migration by Five-Year Age Groups, Hispanic Origin, Race and Sex 2000-2010
}

\author{
Richelle L. Winkler, Department of Social Sciences, Michigan Tech University ${ }^{\mathrm{i}}$ \\ Kenneth M. Johnson, The Carsey Institute, University of New Hampshire \\ Cheng Cheng, Department of Sociology, Princeton University \\ Paul R. Voss, Odum Institute for Research in Social Science, University of North Carolina ${ }^{\mathrm{i}}$ \\ Katherine J. Curtis, Department of Community and Environmental Sociology and Applied \\ Population Laboratory, University of Wisconsin- Madison ${ }^{\mathrm{i}}$ \\ ${ }^{\mathrm{i}}$ Affiliate of the Center for Demography and Ecology, University of Wisconsin- Madison
}

February 2013

\author{
Contact Information: \\ Richelle L. Winkler \\ Department of Social Sciences \\ Michigan Technological University \\ 217 Academic Office Building \\ 1400 Townsend Drive \\ Houghton, MI 49931 \\ Tel: 906-487-1886 \\ E-mail: rwinkler@mtu.edu
}

This research was supported by Grant Number 7R03HD069737-02 from the Eunice Kennedy Shriver National Institute of Child Health and Human Development. Additional support was provided by the U.S. Department of Agriculture, Economic Research Service, Joint Research Agreement No. 58-6000-0-0055. The content is solely the responsibility of the authors and does not necessarily represent the official views of the Eunice Kennedy Shriver National Institute of Child Health and Human Development, the National Institutes of Health, or the United States Department of Agriculture.

The authors wish to express their appreciation to Jim Beaudoin at the Applied Population Laboratory at the University of Wisconsin-Madison for designing an interactive website that makes these data publically available. This website is available at www.netmigration.wisc.edu. In addition, we'd like to thank Dan Veroff of the Applied Population Laboratory, John Cromartie at the USDA Economic Research Service, Warren Brown at the Cornell Institute for Social and Economic Research, Steve Murdoch at Rice University, Laszlo Kulcsar at Kansas State University, and Glenn Fuguitt who is professor emeritus at the University of WisconsinMadison for their assistance and encouragement over the course of this project. 


\title{
County-Specific Net Migration by Five-Year Age Groups, Hispanic Origin, Race and Sex 2000-2010
}

\author{
Richelle L. Winkler, Kenneth M. Johnson, Cheng Cheng, \\ Paul R. Voss, and Katherine J. Curtis
}

\section{Introduction}

This report documents the methodology used to prepare county-level, net migration estimates by five-year age cohorts and sex, and by race and Hispanic origin, for the intercensal period from 2000 to 2010. The estimates were prepared using a vital statistics version of the forward cohort residual method (Siegel and Hamilton 1952) following the techniques used to prepare the 1990 to 2000 net migration estimates (Voss, McNiven, Johnson, Hammer, and Fuguitt 2004) as described in detail below. These numbers (and the net migration rates derivable from them) extend the set of decennial estimates of net migration that have been produced following each decennial census beginning with 1960 (net migration for the 1950s: Bowles and Tarver, 1965; 1960s: Bowles, Beale and Lee, 1975; 1970s: White, Mueser and Tierney, 1987; 1980s: Fuguitt, Beale, and Voss 2010; and 1990s: Voss, McNiven, Hammer, Johnson and Fuguitt, 2004).

The residual method is a straight-forward manipulation of the demographic balancing equation. The basic methodology begins with the fundamental demographic balancing equation:

$$
P_{1}=P_{0}+(B-D)+(I M-O M)
$$

which, upon reorganization of terms, yields:

$$
I M-O M=\left(P_{1}-P_{0}\right)-(B-D)
$$

The equation states that the difference between in-migration (IM) and out-migration $(O M)$ is equal to the population change over the decade $\left(P_{1}-P_{0}\right)$ less the "natural increase" over the decade $(B-D)$. Since $I M$ and $O M$ generally are not measured quantities, we cannot know the difference precisely. We can, however, estimate this difference (by using the terms on the right side of the equation), which, following common convention, we call "net migration" (NM). We assume these right-hand terms either are known or are capable of being well estimated. Thus, net migration is estimated as the residual of the difference between population change and natural increase over an intercensal period.

Equation [2] can be used to estimate net migration for any geographic area for which the right-hand terms are available from reliable sources. (For a comprehensive discussion of the basic method, and the effects of measurement errors in $P_{0}$ and $P_{1}$, see Hamilton, 1966.) Further, depending on the availability and quality of data, net migration based on equation [2] can also be calculated for various age, sex, race and Hispanic origin groups, though the equations become burdened with many subscripts, which we omit for readability. 
The net migration estimates described here were developed using population counts at Census 2000 and 2010 and birth and death counts from the National Center for Health Statistics as basic input data. They do not rely on sampling and are highly accurate and reliable. The net migration residuals are as solid a set of estimates of net migration as possible to generate in the United States without collecting administrative data on moves.

At the same time, it should be clear that the data described here are estimates of net migration. As such, they cannot address inflows and outflows. Net estimates are limited in that they may confound changing migration propensities with changing population stocks, and they cannot depict the important effects of population turnover (they miss the gross flows that often overwhelm any net change), nor can they provide information regarding origins and destinations. Limitations aside, reliable net migration estimates are valid indicators that do capture the ultimate outcomes of how migration changes population composition by age, sex, race, and ethnicity for counties. The dataset described here complements migration flow data available from the Internal Revenue Service (IRS) by providing highly reliable data by detailed demographic characteristics at the county level.

Following a section describing the various uses of county-level net migration estimates, we present a detailed summary of the methodology used to generate the 2000 to 2010 estimates by age, sex, race and Hispanic origin. Finally, some general caveats regarding the use of these estimates are provided. In the six appendices we include (1) a description of our variable naming conventions; (2) tables describing the specific adjustments made to Census 2000 and 2010 enumerated populations; (3) a detailed description of how we handled Broomfield County, Colorado; (4) a table detailing the changes made to Federal Information Processing (FIPS) codes for the purposes of maintaining consistent county boundaries; (5) detailed adjustments to estimates of births in 2009; and (6) a codebook for the electronic dataset.

\section{Uses for County-Level Net Migration Data}

County-level net migration data by demographic characteristics are essential for addressing multiple social and economic research and planning needs. Health care needs, social service demands, the size and character of the proximate labor market, demand on the local infrastructure, housing markets, and environmental impacts are all affected by changes in population composition induced by differential age-specific migration. Furthermore, researchers must be able to connect specific migration outcomes with local socioeconomic conditions to investigate the community level causes and consequences of migration.

Important research examining the extent to which natural increase or migration of particular populations is fueling population growth (or decline) in different types of places and among different age groups is impossible without place- and age-specific migration data. This includes foundational work such as Keyfitz's (1980) analysis of the impact of natural increase and migration on urbanization patterns and Plane et al.'s (2005) investigation of migration patterns up and down the urban hierarchy at different ages. It also concerns emerging work on the degree to which natural increase and migration are fueling Hispanic population growth in new destinations (Johnson and Lichter 2008). Similarly, continuing work examining the impact of baby boom migration on population redistribution as these large cohorts enter their retirement 
years (Cromartie and Nelson 2009) and research investigating the consequences of migration on black-white differences in southern county poverty rates (Curtis and DeWaard 2010) cannot be maintained without high quality small area migration data by age, sex, and race/ethnicity for 2000 to 2010 .

Previous county level net migration estimates by age (1950-2000) have been used extensively by demographers to examine patterns of net migration (Johnson, et. al 2005; Gibson, Fuguitt and Voss 1996; Johnson and Fuguitt 2000), elderly population change (Fuguitt, Gibson, Beale, and Tordella 1998; Bowles and Beale 1993; Lichter, Fuguitt, Heaton, and Clifford 1981), life cycle migration (Heaton, Clifford, and Fuguitt 1981; Muesser, White and Tierney 1988), the age composition of the population (Clifford, Heaton, Lichter, and Fuguitt 1983; Fuguitt and Heaton 1995, Winkler et al. 2011), and the role of migration in the growth of amenity and retirement areas (Johnson and Stewart 2005).

Applied demographers rely extensively on reliable county-level net migration estimates by detailed demographic characteristics in generating population estimates and projections and for informing health care planners and other service providers who must prepare for future needs. For instance, local health care providers must know if migration is bringing females at prime childbearing age into the community to increase pre- and post-natal physician staffing. Similarly, state-level health policy-makers must understand how the geographic distribution of the elderly population is changing to ensure services that meet their needs are available in the appropriate locations. Furthermore, these net migration estimates are critical to applied demographers and planners for producing population estimates and projections that incorporate local migration data directly in their estimates or projections. County and city governments use them for projections relative to the construction of water and sewer, municipal power, and general economic development projects. They are used by multicounty entities for planning in the areas of transportation, water development, electric power need assessments, and economic development. Many school districts use them in siting school campuses and in projecting school enrollment. Note: Practitioners using these net migration estimates to inform cohort component population projection should refer to the section beginning on page 12 for important stipulations.

\section{Detailed Methods and Data Sources}

Voss et al. (2004) offer a detailed comparison between three methodological approaches to generating residual based net migration estimates, including census survival rate, life table survival rate, and vital statistics methods. They explain that the survival rate methods have been used historically in cases where reliable birth and death data by detailed demographic characteristics have not been available for local areas (including those produced by Bowles and Tarver, 1965; Bowles, Beale and Lee, 1975; White, Mueser and Tierney, 1987; and Fuguitt, Beale, and Voss 2010), and they demonstrate that when high quality vital statistics data are available, this approach yields reliable estimates (Voss et al. 2004). The net migration estimates we produced for 2000-2010 follow the vital statistics method as employed by Voss et al. (2004), because detailed mortality and fertility records were made available through a restricted use agreement from the National Center for Health Statistics (NCHS) through the Vital Statistics Cooperative Program. These data files permit the aggregation of deaths and births for each county in the U.S. by single year of age, sex, race, and Hispanic origin. With this fine level of mortality and fertility detail available, there was no need to estimate mortality rates using census survival or life table methods. 
Equation 3 further specifies Equation 2 (shown above) to specifically address Net Migration (NM) using the vital statistics method used to construct the 2000-2010 net migration estimates reported here. $P_{0}$ refers to the starting population observed at Census 2000 by county, age, sex, race, and Hispanic origin and adjusted for identified census errors and for estimated undercount/overcount. $P_{1}$ refers to the final population observed at Census 2010, adjusted for estimated undercount/overcount. $B$ and $D$, respectively, refer to observed (2000-2008) and estimated (2009-2010) birth and death data recording individual births and deaths by single year of age, sex, race, Hispanic origin, and county of residence as collected through state level birth and death certificates and reported by the National Center for Health Statistics (NCHS). Each of these data sources and the organizational processes involved are described in more detail below.

$$
N M=\left(P_{1}-P_{0}\right)-(B-D) \quad[3]
$$

Where:

$P_{1}=$ final population (adjusted) observed at Census 2010

$P_{0}=$ starting population (adjusted) observed at Census 2000

$B=$ number of births 2000-2010, aggregated by county of residence

$D=$ number of deaths 2000-2010, aggregated by county of residence

Assuming zero net migration, Equation 3 can be re-arranged to calculate the expected population in 2010, which represents what the 2010 population $\left(P_{1}\right)$ would have been absent net migration (see Equation 4). County-specific expected populations in 2010 (EP) are derived for cohorts by aging the 2000 population forward in time, adding births and subtracting deaths. In other words, the expected population of cohorts ages 10 to 99 and ages 100 and over in 2010 is calculated as the base 2000 population minus deaths occurring to persons of that cohort advanced ten years in age. For cohorts born during the 2000s (ages 9 and under in 2010), expected population is the difference between births and deaths experienced by that cohort in the 2000s, advanced in age from the date of birth. These cohort specific expected populations represent the number of people expected to be alive and enumerated in the 2010 census absent net migration.

$$
E P_{1}=P_{0}+B-D
$$

Net migration can then be estimated as the difference between the observed final population $\left(P_{1}\right)$ and the expected population $\left(E P_{1}\right)$, by substituting Equation 4 into Equation 3 . The net migration rate (per 100 individuals) is calculated by dividing the estimated number of net migrants by the expected population, as shown in Equation 6.

$$
\begin{aligned}
& N M=P_{1}-E P_{1} \\
& N M R=\left(N M / E P_{1}\right) * 100
\end{aligned}
$$

Unless otherwise noted, cohorts are county-level and specific to age (single years of age through 84 and then a group age 85 and over), sex, race, and Hispanic origin. For our purposes, a year spans the 12 month interval from April 1 to March 31 - dates chosen because the Census Bureau nominally enumerates the population on April 1. Thus, persons born in 2000 refer to those born between April 1, 2000 and March 31, 2001. In reporting the net migration results, we aggregated single years of age through 84 into five-year age groups. 
Though the process of constructing net migration rates, we organized total population into the following five race/ethnic groups: (1) Hispanic, (2) Non-Hispanic White (NH white), (3) Non-Hispanic Black (NH black), (4) Non-Hispanic American Indian or Alaskan Native (NH AIAN), and (5) Non-Hispanic Asian or Native Hawaiian/Pacific Islander (NH Asian). In reporting the net migration results, we aggregated the $\mathrm{NH}$ AIAN and $\mathrm{NH}$ Asian groups into $\mathrm{NH}$ Other, because the populations in these groups were small in a large number of counties, creating inconsistent and difficult to interpret net migration rates.

\section{Census 2000: The Starting Population}

Net migration estimates are sensitive to misallocations, specificity of race/ethnic classification, and to undercount and overcount in the populations on which the estimates are based. Great care was taken to adjust the 2000 and 2010 enumerated Census populations toward our best guess of the "true" populations, based on the Census Bureau's error corrections and estimates of net undercount/overcount. These re-organized and adjusted population counts are used to calculate net migration numbers and rates. The specific adjustments we made are discussed in detail below.

In constructing net migration estimates for the 1990s, Voss et al. (2004) made specific adjustments for undercount/overcount to Census 1990 and Census 2000 data. They did not, however, have the full set of Census 2000 evaluation studies or the results of the Count Question Resolution program available at the time of publication. For this reason, our team did not use for a starting population the same dataset that Voss et al. (2004) used for a final population. Instead, we relied on the US Census Bureau's Census Estimates Base to account for known errors, and we adjusted for undercount/overcount following the reports of ACE Revision II and Demographic Analysis, as described below.

Census 2000 included several misallocations that affected county population enumerations. These were subsequently recognized through the Census Bureau's Count Question Resolution program (US Census Bureau 2005). The standard census data products do not reflect these corrections, but they have been incorporated into the Census Bureau's intercensal population estimates program. For this reason, we use the resident population estimates base from census day (4/1/2000) as published through the US Census Bureau's Population Estimates program rather than the enumerated 2000 population reported in standard census data products. More specifically our starting population is from the Annual County Resident Population Estimates by Age, Sex, Race, and Hispanic Origin (US Census Bureau 2009a). While these data correct for misallocations, they require further organization and adjustment into consistent race/ethnic groups, single year of age, and to deal with undercount/overcount.

\section{Race and Ethnicity Classification}

The intercensal estimates assign persons reporting "Some Other Race" as their race on the census questionnaire to an OMB race, including White, Black or African American, American Indian or Alaskan Native, Asian, or Native Hawaiian or Pacific Islander (see US Census Bureau 2009b for complete description of the categories and how they are assigned). We 
grouped the Asian and Native Hawaiian or Pacific Islanders into a single category because of the small number of Native Hawaiian or Pacific Islander persons in most counties. The data include these racial groupings but divide them into two categories- those who identify as each race "alone" and a group for that race "alone or in combination." In addition, the dataset includes a category of people identifying with "two or more races." For our purposes, anyone with Hispanic ethnicity was considered Hispanic despite the racial classification so that Hispanic ethnicity trumped all race categories. Non-Hispanic populations recorded as White, Black or African American, American Indian or Alaska Native (AIAN), Asian or Native Hawaiian or Pacific Islander (Asian) "alone" were classified in these respective groups.

To be consistent with the net migration estimates produced in prior decades (especially those for the 1990s) and because of the small number of people identifying as two or more races in most counties, we allocated persons recording two or more races among the other race groups based on the following steps and assumptions. For each race group (NH White, NH Black, NH AIAN, and NH Asian), we estimated the number of people who identified as that race in combination (combo counts) with some other race by subtracting the "alone" population from the population "alone or in combination". Next we estimated the total number of combinations by summing the combo counts across all racial groups (combo sum). We then calculated the percent of the combo sum that each race group comprised and multiplied this proportion by the population of two or more races to estimate the combination population to add to each race "alone" group. Our base population then, by non-Hispanic race, includes the alone population and an estimate of the combination population that identifies at least partially as that racial group. Essentially, this means that our race/ethnic classifications divide the combination race population by distributing portions of persons to each specific racial group with which they identify. Though the initial dataset and processes differ, the concept of dividing the combination race population follows that used by Voss et al. (2004) in generating the net migration estimates for the 1990s.

\section{Adjusting for Undercount/Overcount}

All US censuses prior to 2000 faced population undercount, particularly among specific subgroups including minorities, children, and renters (Clark and Moul 2004). The Census Bureau conducts follow-up coverage studies to estimate undercount . Following Census 2000, these included the initial Accuracy and Coverage Evaluation (ACE 2001), the Accuracy and Coverage Evaluation Revision II (ACE Revision II, 2003), and comparisons with estimates generated using administrative records through Demographic Analysis (DA, Robinson 2001). For the first time, Census 2000 returned a net overcount of the total population, according to ACE and ACE Revision II and only a $0.12 \%$ net undercount as compared to DA (Clark and Moul 2004). Yet while the overall net undercount at Census 2000 was negligible, Census 2000 coverage varied significantly by age and race with undercount among children, overcount amount college age and retirement age adults, and an estimated 2-3\% undercount of Blacks (Clark and Moul 2004, Citro et al. 2004).

Because census undercount varies significantly by age, sex, race, and ethnicity, we made adjustments to the Census 2000 data by these specific cohort groups. For the population age 10 and over, we increased the census estimates base by the percent undercount reported by ACE 
Revision II and summarized in the National Research Council's review of Census 2000 (see Citro et al. 2004, p. 255). It should be noted that for some age groups, the estimate of net undercount was negative (indicating a net overcount). There were no specific estimates of undercount by ethnicity, age, and sex, but findings suggest that Hispanics were counted at more similar rates as blacks than non-Hispanic whites, who comprise the dominant group in the nonblack category (Citro et al. 2004). For this reason, we assumed that Hispanic undercount/overcount followed black estimates, rather than nonblack, and we assume that all race/ethnic groups other than black and Hispanic follow nonblack rates. Estimates of undercount/overcount by age, sex, race/ethnicity were not available for any geographic specificity other than the nation as a whole. We applied these national estimates (by age, sex and race/ethnicity) to each of the county cohort populations.

For the population under age 10, Demographic Analysis (DA, ) provides a more reliable estimate of census undercount (Voss et al. 2004, Robinson 2001, O'Hare et al. 2012), as DA are constructed based on administrative records, including birth and death certificates. We rely on the Revised Demographic Analysis estimates released in September 2001 by race, sex, and age (see Robinson 2001) to adjust the population under age 10. These are reported by the National Research Council review of Census 2000 in the Demographic Analysis Appendix, Table 2 (Citro et al. 2004). Again, we assume that the Hispanic population undercount follows the black population undercount (rather than nonblack) because like the ACE Revision II, DA only includes estimates by black/nonblack, and that NH white, NH AIAN, and NH Asian groups follow nonblack rates. Specific adjustments are summarized in Appendix 2.

The final step was to disaggregate the data into single years of age, until age 85 after which the population is grouped into an 85 plus age group. Using the age distribution reported from the standard enumerations in US Census 2000 (SF1) on population counts by single year of age, sex, race, and ethnicity; we calculated the proportion of each five-year age group that were at each single year of age. We then multiplied this proportion by our adjusted five-year age group estimates base data (essentially the Census 2000 data corrected for misallocations and adjusted for undercount/overcount). For all age groups except 0-4, we assume that the adjustment for overcount/undercount is evenly distributed across single years of age. But research suggests that undercount is greatest among very young children (O'Hare et al. 2012, Siegel 1974, Anderson and Silver 1985). Following the process used by Voss et al. (2004) to adjust the 2000 population, we distributed the estimated undercount among the five year age group $0-4$ as follows. Those under age 1 were allocated $27.1 \%$ of the undercount, $24.3 \%$ went to those age $1-2,23.6 \%$ to those age $2-3,15.5 \%$ to those age $3-4$, and $9.5 \%$ to those age $4-5$.

The result of this process was a dataset including population counts by single year of age, sex, race, and Hispanic origin corrected for misallocations and adjusted for undercount/overcount for all US counties. This is the starting population we used to calculate net migration.

\section{Census 2010: The Final Population}

Like with the starting population, we relied on the US Census population estimates base for $4 / 1 / 2010$ as our base final population. Specifically, the file is CC-EST2011-ALL DATA- 
[ST-FIPS]: Annual County Resident Population Estimates by Age, Sex, Race, and Hispanic Origin: April 1, 2010 to July 1, 2011, released in May 2012 (US Census Bureau Population Division 2012). At the time of its release the Census 2010 Count Question Resolution program had not identified any misallocations that would affect county level population, and so this file does not correct for any potential enumeration misallocations. The file does, however, group the enumerated population at Census 2010 into the same race, ethnic, age, and sex categories described above for the starting population. Identical processes to those described above for Census 2000 were used to organize the 2010 population into race/ethnic categories for analysis. Maintaining consistency in the starting and final populations is vital for generating reliable net migration estimates.

\section{Adjusting for Undercount/Overcount}

Comparing the enumeration results from Census 2010 with Demographic Analysis (US Census Bureau 2012) and the US Census Bureau's Census Coverage Measurement survey (CCM), indicates no statistically significant net undercount or overcount. There are, however, indications that certain age, sex, race, and ethnic groups were under- or over- counted. CCM, for instance, shows a $2 \%$ net undercount for blacks, $1.5 \%$ net undercount for Hispanics, $4.9 \%$ net undercount of AIANs, and a $0.8 \%$ net overcount among non-Hispanic whites (Mule 2012). Demographic Analysis (DA) also shows about a 2\% net undercount for blacks, but when disaggregated by sex suggests that black males were undercounted by about $3.5 \%$ while black females were overcounted by about $1.5 \%$. Among nonblacks, DA results again mimic CCM noting a $0.8 \%$ net overcount, but with divergent patterns between males and females with nonblack females being overcounted by about $1 \%$ and males undercounted by about $0.4 \%$.

By age, CCM indicates statistically significant undercount among young children of both sexes (age <5), males age 18-29, and males age 30-49. Net overcount is indicated among children age 10-17, females age 30-39, males over age 50, and females over age 50 (Mule 2012). CCM did not find any state with statistically different than zero net undercount/overcount, but at the state level breakdowns by age, race, and sex are not available. In sum, results from CCM and DA suggest that net undercount among some age, sex, race, ethnic categories cancelled out net overcount among other groups to produce a total net undercount/overcount of zero. At the same time, however, age/sex/race/ethnic -specific undercounts/overcounts were even greater among some groups than at Census 2000, indicating adjustments for the purposes of this project are necessary.

DA provides specific estimates of the population by single year of age, sex, by black/nonblack race and for Hispanic ethnicity among children. As described above, DA and CCM results are similar. The reliability of DA data have been significantly improved over the last several years with computing advancements, electronic records, and evaluative analysis; and they are particularly sound for estimating the child population (Robinson 2001, O'Hare et al. 2012). For these reasons, we primarily rely on estimates of undercount/overcount generated by calculating the rate of difference between DA and the census enumerated population to adjust the Census 2010 final population, when CCM suggests there are statistically significant undercounts/overcounts. DA estimates are not specifically available for NH whites, AIANs or for Asians, and are only available for Hispanics at ages 0-19. Because CCM indicates more undercount of AIANs than of blacks, we apply the undercount rate for black males to AIAN 
males, and $1 / 2$ the undercount rate for black males to AIAN females. Because CCM does not show any significant net undercount/overcount of Asians, we do not adjust this population. CCM indicates about half a percentage point less undercount for Hispanics than blacks. For the Hispanic population under age 20 (where DA estimates are available), we rely on DA estimates to adjust the population. For Hispanics age 20 and over, we assume the undercount rate of Hispanic males to be $25 \%$ less than that of black males and the undercount rate of Hispanic females to be $200 \%$ less than black males. Finally, we assume that non-Hispanic white undercount/overcount rates are slightly higher than for the nonblack group as a whole, which would include Hispanics and AIANs. Altogether, adjusting the populations in these ways cumulatively produces net undercount/overcount rates similar to the CCM estimates.

Appendix 2 shows the specific adjustments made. These adjustments were multiplied by the census estimates base population (4/1/2010) to generate the final population at Census 2010 by five-year age groups used in the construction of net migration estimates.

\section{County Boundaries}

Since the boundaries of some counties changed between 2000 and 2010, we aggregated certain counties to county groups to ensure continuity in boundaries across censuses. In Virginia, this involved integrating Clifton Forge independent city into Alleghany County. In Colorado, the creation of Broomfield County in 2001 from parts of Adams, Boulder, Jefferson, and Weld Counties challenged our team to separate Broomfield County data at Census 2000 and in the birth and death data from NCHS from the prior constituting counties so that net migration estimates for Broomfield County could be generated, even though this county did not exist at Census 2000. A detailed description of how we handled Broomfield County is found in Appendix 3. Finally, three county aggregation groups were constructed in Alaska to deal with county boundary changes. We aggregated (1) Denali Borough, Yukon-Koyukuk Census Area, and Southeast Fairbanks Census Area; (2) census geographies around Ketchikan, Petersburg, Wrangell City, Hyder, and Prince of Wales; and (3) Skagway Municipality with Hoonah-Angoon Census Area. The specific county groupings and associated FIPS codes changes are detailed in Appendix 4.

\section{Births}

As mentioned above, annual birth microdata files for the years 1999 through 2008 were made available to us under a restricted use agreement with the NCHS. These included individual level birth records with data on county and month of birth, sex, race of mother and father, and Hispanic origin of mother and father for each year 2000-2008. The county codes used in Natality Detail Files 2000-2003 are based on the 1990 Census definition, while county codes for 2004-2008 are based on the 2000 Census definition. We deal with this issue by aggregating to county groups where necessary, as described above and in Appendix 3 regarding Broomfield, Adams, Boulder, Jefferson, and Weld counties in Colorado. We followed the process described below to assign race/ethnic status (Hispanic, NH white, NH black, NH AIAN, or NH Asian) to these births. Finally, we aggregated the births by sex and race/ethnicity to counties and grouped them into single year age cohorts based on their date of birth.

We imputed race/ethnicity of births primarily based on the reported race/ethnicity of the mother. As with Census 2000 and Census 2010 data, we classified anyone reporting Hispanic ethnicity as Hispanic, regardless of race. There were, however, an average of $0.6 \%$ of all records 
where data on mother's race or ethnicity was missing. In these cases, we imputed based on the father's reported race/ethnicity. In cases where neither the mother or father's ethnicity is known and the reported race is Black, AIAN, or Asian, we assume that the birth is non-Hispanic and falls within the respective race group. If the Hispanic origin is unknown and the race is White, we assume that a proportion of those births are Hispanic. This proportion is determined for each county individually, based on the proportion of women living in the county in their childbearing years (age 15-44) who are Hispanic, as identified in the US Census Population Estimates for July 1, 2005. More specifically, we follow the formulae below for each county.

$$
\begin{aligned}
& \mathrm{B}_{\mathrm{h}}=\mathrm{B}_{\mathrm{H}}+\left(\mathrm{HF}^{(15-44)} / \mathrm{F}^{(15-44)}\right) * \mathrm{~B}_{\mathrm{u}} \\
& \mathrm{B}_{\mathrm{w}}=\mathrm{B}_{\mathrm{W}}+\left(1-\left(\mathrm{HF}^{(15-44)} / \mathrm{F}^{(15-44)}\right)\right) * \mathrm{~B}_{\mathrm{u}}
\end{aligned}
$$

Where

$$
\begin{aligned}
& \mathrm{B}_{\mathrm{h}}=\text { Final estimate of Hispanic births } \\
& \mathrm{B}_{\mathrm{w}}=\text { Final estimate of Non-Hispanic White births } \\
& \mathrm{B}_{\mathrm{H}}=\text { Reported Hispanic births } \\
& \mathrm{HF}^{(15-44)}=\text { Hispanic females, age } 15-44 \\
& \mathrm{~F}^{(15-44)}=\text { Total females, age } 15-44 \\
& \mathrm{~B}_{\mathrm{u}}=\text { Births of unknown ethnicity and white race }
\end{aligned}
$$

Hispanic fertility rates are higher than non-Hispanic whites and so the proportion of Hispanic births may be higher than the proportion of Hispanic women. At the same time, however, a disproportionate share of the missing ethnicity births may be non-Hispanic, because Hispanics may be more likely to have and record a salient ethnicity. While the higher Hispanic birth rate would suggest our methods above would underestimate Hispanic births, a disproportionate share of non-Hispanic missing ethnicity births would overestimate Hispanic births. We expect that error associated with each of these two dimensions would essentially cancel one another out and so we do not specify either in the formulae above.

Because detailed natality files for 2009 and 2010 were not yet available at the time these net migration estimates were produced, we estimated the number of county level births by race/ethnicity for 2009 and for the first quarter of 2010. National level records of births by race and Hispanic origin were available for 2009 and 2010 and show that the total number of births in the US declined by $2.75 \%$ between 2008 and 2009 and by an additional 3.2\% between 2009 and 2010 (Martin et al. 2012). National birth data by race/ethnicity show that all groups experienced decline in the number of births, but that Hispanic births declined about twice as much as nonHispanics (Sutton et al. 2011). The process to estimate county-level births by race/ethnicity (described below) was based upon conforming to these national patterns, and we raked county estimates to approximately sum to these total national birth reports.

Because our cohorts were organized based on the census year (April 1 to March 31), we needed to estimate the number of births that occurred in the first quarter of 2009 to complete our total estimate of births for the 2008 census year. In 2008, the ratio of births that occurred January to March to those that occurred April to December was 0.3265. Because we know that births declined between 2008 and 2009, we assume this ratio would be slightly lower (0.3194) if we 
compared January to March 2009 with April to December 2008. We then use this ratio to add an estimate of the number of births in the first quarter of 2009 to our cohort year 2008 birth totals. We do this by multiplying observed births in April to December 2008 in each county by 1.3194 . The assumption behind this approach is that monthly patterns of births observed in 2008 continued in 2009, but that the number of births declined somewhat between 2008 and 2009, as observed in the national data.

Next, we estimated the number of births that occurred April 1, 2009 to March 31, 2010 (our cohort year 2009) in each county by race/ethnicity and sex. Here, we generally assumed that births in each county decreased more between 2008 and 2009 than they did between 2007 and 2008, following national patterns. We calculated the percent change in the number of births between 2007 and 2008 for each county by race/ethnicity and sex, and we applied this rate of increase to the 2008 birth estimates to generate a preliminary estimate of births for 2009 . For counties (and race/ethnic groups) where this rate of change was greater than $1.0(100 \%--$ instances of small minority populations) we held the rate to $100 \%$. We then adjusted these preliminary estimates downward, because national level data indicated a faster rate of decline between 2008 and 2009 than between 2007 and 2008 (Sutton et al. 2011). Specific adjustments were made by race/ethnicity following observed national level differences. Asians were not further adjusted. Blacks were adjusted downward by $1.5 \%$. Hispanics were adjusted downward by $4.4 \%$. AIAN were adjusted downward by $0.2 \%$. Whites were adjusted downward by $1.62 \%$. We found the sum of these county level estimates to closely reflect reported national births by race/ethnicity.

To identify any unrealistic estimates, we conducted several tests. We compared our estimates against observed births in prior decades and with US Census Population Estimates program birth estimates, and we reviewed crude birth rates by race/ethnicity. Our team checked estimates of concern against state published data (where available) and made substitutions where necessary. This process revealed 50 counties where Hispanic births were adjusted, 22 counties where Asian births were adjusted, 43 counties where Black births were adjusted, 34 counties where AIAN births were adjusted, and 160 counties where White births were adjusted. Adjusted counties are listed in Appendix 5.

\section{Deaths}

The steps to create death count estimates are similar to that of births. Death records were obtained from NCHS's Multiple Cause of Death Files 2000-2008 through a restricted data use agreement. These records contain data on race, Hispanic origin, sex, month of death as well as age and county of residency at death. As with the birth records, a few death records had unknown Hispanic origin. We dealt with these records with the following steps. Persons reported as Black, AIAN, or Asian are assumed to be non-Hispanic and were assigned to respective race category. For White persons of unknown Hispanic origin, we assumed that deaths of unknown Hispanic origin were non-Hispanic or Hispanic in the same proportion as deaths of known Hispanic origin.

Age at death was also important for constructing net migration estimates, and some of the death records were missing data on age. Deaths at unknown ages were imputed according to the share at which deaths at known ages occur by age, sex, race/ethnicity, and county. There were very few records with missing data on age at death. 
We then estimated deaths for the first quarter of 2009 (to complete our cohort year deaths 2008) and for our cohort year 2009 (April 1, 2009 to March 31, 2010). In 2008, the ratio of deaths that occurred January to March to those that occurred April to December was 0.3698. We used this ratio to add an estimate of the number of deaths in the first quarter of 2009 to our cohort year 2008 deaths by multiplying observed deaths in April to December 2008 by 1.3698 . The assumption behind this approach is that monthly patterns of deaths (by age, sex, race/ethnicity and county) observed in 2008 continued in 2009. Finally, we estimated deaths in 2009 by simply assuming that they were equal to the number of deaths observed in 2008 by age, sex, race/ethnicity and county.

\section{Quality Control}

In order to test the quality of the net migration estimates, our team shared preliminary estimates with knowledgable state demographers, researchers, and with county extension educators. We asked these experts to critically review the estimates (in comparison to estimates for prior decades) for counties and states for which they are familiar, and to report on any suspect patterns. We did not receive any indication of problems. In addition, we compared our aggregate net migration estimates (age, sex, race, and ethnicity summed together) to annual estimates of migration published by the IRS (inflows minus outflows= net migration) summed together to generate a comparable decade interval. We found that our estimates differed, on average, from the IRS-based estimates by about 3.7\% (mean absolute percent error, or MAPE). Where our estimates differed more significantly, were counties with universities where IRS estimates are known to miss college students who are claimed by their parents on tax forms. In sum, these controls offer confidence to the accuracy of the data described here.

\section{Website}

These data are publically available for download and for interactive analysis on the web at www.netmigration.wisc.edu. This site integrates the 2000-2010 estimates with age-specific net migration estimates for prior decades, 1950-2000. It allows for custom map and chart building making for easy on-the-fly data analysis. In addition, users may create custom data downloads of net migration data 1950-2010 and associated census data 1990, 2000, and 2010, and they may download these data for different types of counties (metro vs. nonmetro or following USDA Economic Research Service county typologies).

\section{Using the 2000-2010 Net Migration Estimates for Cohort Component Projections}

For those who would use these estimates in the process of creating cohort component projections, it is important to note how these estimates gleaned from a vital statistics (VS) approach are substantively different than net migration estimates that employ survival rates, such as those produced in the 1950s through the 1980s. Our methods are equivalent to those used by Voss et al. (2004) in the 1990s and a more full description of these differences can be found at the documentation for those data (see Voss et al. 2004).

In a typical projection model using net migration rates, an expected population is first derived for the end point in a projection cycle, then net migration is calculated for the interval by applying the net migration rate (NMR by age, sex, and race) to the expected population. The 
estimated amount of net migration is then added to the expected population (by age, sex and race) and this population then constitutes the beginning population for the next cycle of the projection model. This procedure, unfortunately, does not hold precisely when the VS methodology has been used to estimate net migration, for the numerator includes deaths to inMigrants, and the denominator, or expected population, has already been decremented by the number of deaths to in-Migrants. In a projection model, it would be useful to estimate the number of deaths to in-Migrants in base cycle and adjust the net migration rate. For details on how to complete this process see Voss et al. (2004). 


\section{References}

Anderson, Barbara A., and Brian D. Silver. 1985. Estimating census undercount from school enrollment data: An application to the Soviet censuses of 1959 and 1970. Demography 22(2):289-308.

Bowles, Gladys K., Calvin L. Beale, and Everett S. Lee. 1975. Net Migration of the Population, 1960-1970, by Age, Sex, and Color. Athens, GA: University of Georgia Printing Department.

Bowles, Gladys K., and James D. Tarver. 1965. Net Migration of the Population, 1950-1960 by Age, Sex and Color. Washington, DC: United States Department of Agriculture.

Bowles, G. K., C. L. Beale. 1993. The changing concentration of the older nonmetropolitan population, 1960-1990. Journal of Gerontology: Social Sciences 48:S278-S288.

Citro, C.F., D. L. Cork, and J. L. Norwood (Eds.). 2004. The 2000 Census: Counting under Adversity. Panel to Review the 2000 Census, National Research Council. The National Academies Press.

Clark, J.R. and D. A. Moul. 2004. Census 2000 Testing, Experimentation, and Evaluation Program Topic Report No. 10, TR-10, Coverage Improvement in Census 2000 Enumeration, U.S. Bureau of the Census.

Clifford, W. B., T. B. Heaton, D. T. Lichter, and G. V. Fuguitt. 1983. "Components of Change in the Age Composition of Nonmetropolitan Americans." Rural Sociology 48: 458-470.

Cromatie, J. and P. Nelson. 2009. Baby boom migration and its impact on rural America. Economic Research Report Number 79: U.S. Department of Agriculture Economic Research Service.

Curtis, K. J. and J. DeWaard. 2010. "Compositional Threat? An Analysis of Return Migration and Southern Poverty." Paper presented at the annual meeting of the Population Association of America, Dallas, TX, April 15 $5^{\text {th }}-17^{\text {th }}, 2010$.

Fuguitt, Glenn V., and Calvin L. Beale. 1993. "The Changing Concentration of the Older Nonmetropolitan Population 1960-1990.” Journal of Gerontology: Social Sciences 48: S278-S288.

Fuguitt, G. V., C. L. Beale, and P. R. Voss. 2010. County-Specific Net Migration Estimates, 1980-1990. ICPSR26761-v1. Ann Arbor, MI: Inter-university Consortium for Political and Social Research, 2010-04-02. doi:10.3886/ICPSR26761

Fuguitt, G. V., R. M. Gibson, C. L. Beale and S. J. Tordella. 1998. "Elderly population change in nonmetropolitan areas: from the turnaround to the rebound." Paper presented at the annual meeting of the Western Regional Science Association, Monterey, California, February 22.

Fuguitt, G. V. and T. B. Heaton. 1995. The impact of migration on the nonmetropolitan population age structure. Population Research and Policy Review 14:215-232.

Gibson, Richard M., Glenn V. Fuguitt and Paul R. Voss. 1996. Net Migration by Age for Wisconsin Counties, 1950-1990. Madison, WI: University of Wisconsin-Madison Department of Rural Sociology Applied Population Laboratory. Population Series 90-5. 
Hamilton, C. Horace. 1966. "Effect of Census Errors on the Measurement of Net Migration." Demography 3:393-415.

Heaton, T. B., W. B. Clifford and G. V. Fuguitt. 1981. "Temporal shifts in the determinants of young and elderly net migration in nonmetropolitan areas." Social Forces 60:41-60.

Johnson, K.M. and S.I. Stewart. 2005. “Amenity Migration to Urban Proximate Counties.” pp. 177-196 in G. P. Green, D. Marcouiller and S. Deller (eds.), Amenities and Rural Development: Theory, Methods and Public Policy. Cheltenham, UK: Edward Elgar Publishing.

Johnson, K. M., P. R. Voss, R. B. Hammer, G. V. Fuguitt, and S. McNiven. 2005. "Temporal and Spatial Variation in Age-Specific Net Migration in the United States." Demography 42:791-812.

Johnson, K. M, and D. T Lichter. 2008. "Natural increase: a new source of population growth in emerging Hispanic destinations in the United States." Population and Development Review 34:327-346.

Johnson, K. M, and G. V Fuguitt. 2000. "Continuity and Change in Rural Migration Patterns, 1950-1995*." Rural Sociology 65:27-49.

Keyfitz, N. 1980. "Do cities grow by natural increase or by migration?" Geographical Analysis 12 (2): 142-156.

Lichter, D. T., G. V. Fugitt, T. B. Heaton and W. B. Clifford. 1981."Components of Change in the Residential Concentration of the Elderly Population: 1950-1975.” Journal of Gerontology 36:480-489.

Martin, J.A., B.E. Hamilton, S.J. Ventura, M.J.K. Osterman, E.C. Wilson, and T.J. Mathews. 2012. Births: Final data for 2010. National Vital Statistics Reports 61(1).

Mueser, P. R., M. J. White and J. P. Tierney, 1988. Patterns of net migration by age for U.S. counties 1950-1980: The impact of increasing spatial differentiation by life cycle. Canadian Journal of Regional Science 11:57-76.

Mule, T. 2012. DSSD 2010 CENSUS COVERAGE MEASUREMENT MEMORANDUM SERIES \#2010-G-01. Washington, DC: US Census Bureau.

National Center for Health Statistics. [Name of data file(s)] ([year(s]), as compiled from data provided by the 57 vital statistics jurisdictions through the Vital Statistics Cooperative Program.

O'Hare, W.P. 2012. The Net Undercount of Children in the 2010 U.S. Decennial Census. Paper presented at the Applied Demography Conference in San Antonio, Texas, January 8-10, 2012.

Plane, D. A., C. J. Henrie, and M. J. Perry. 2005. "Migration up and down the urban hierarchy and across the life course." Proceedings of the National Academy of Sciences of the United States of America 102:15313-15318.

Robinson, J.G. 2001. ESCAP II: Demographic Analysis Results. Executive Steering Committee for A.C.E. Policy II, Report No. 1, October 13, 2001. Washington, DC: U.S.Census Bureau. 
Siegel, Jacob S. 1974. "Estimates of Coverage of the Population by Sex, Race, and Age in the 1970 Census.” Demography 11(1):1-23. Retrieved February 27, 2013.

Siegel, Jacob S. and C. Horace Hamilton. 1952. "Some Considerations in the Use of the Residual Method of Estimating Net Migration." Journal of the American Statistical Association 47:475-500.

Sutton, P.D., B.E. Hamilton, and T.J. Matthews. 2011. Recent decline in births in the United States, 2007-2009. NCHS Data Brief, No. 60.

U.S. Census Bureau. 2001. 2000 Census of Population and Housing: Summary File 1 United States. Washington, DC: U.S. Census Bureau.

U.S. Census Bureau. 2005. Count Question Resolution Factsheet: Notes and Errata for Census 2000. Washington, DC: U.S. Census Bureau.

U.S. Census Bureau. 2009a. Annual County Resident Population Estimates by Age, Sex, Race, and Hispanic Origin (vintage 2009). Washington, DC: U.S. Census Bureau.

U.S. Census Bureau. 2009b. Methodology for the State and County Resident Population Estimates by Age, Sex, Race, and Hispanic Origin (vintage 2009): April 1, 2000 to July 1, 2009. Washington, DC: U.S. Census Bureau.

U.S. Census Bureau Population Division. 2012. Annual County Resident Population Estimates by Age, Sex, Race, and Hispanic Origin: April 1, 2010 to July 1, 2011. Washington, DC: U.S. Census Bureau.

U.S. Census Bureau. 2012. Revised 2010 Demographic Analysis Estimates, Released May 2012. Washington, DC: U.S. Census Bureau. Web. [Accessed August 1, 2012]. $<$ http://www.census.gov/popest/research/demo-analysis.html $>$

U.S. Department of Health and Human Services, National Center for Health Statistics. 20002008a. Natality Detail Data, 2000-2008. Hyattsville, MD: U.S. Department of Health and Human Services, National Center for Health Statistics.

U.S. Department of Health and Human Services, National Center for Health Statistics. 20002008b. Multiple Cause of Death of ICD- 9 Data, 2000-2008. Hyattsville, MD: U.S. Department of Health and Human Services, National Center for Health Statistics.

Voss, P. R., S. McNiven, R. B. Hammer, K. M. Johnson, G. V. Fuguitt. 2004. County-specific net migration by five-year age groups, Hispanic origin, race and sex 1990-2000. CDE Working Paper No. 2004-24. Center for Demography and Ecology, University of Wisconsin-Madison. Madison, WI.

White, M. J., P. Mueser, and J. P. Tierney. 1987. Net Migration of the Population of the United States 1970-1980, by Age, Race and Sex: United States, Regions, Divisions, States and Counties [machine-readable data file]. File available from ICPSR, University of Michigan. Ann Arbor, MI: Interuniversity Consortium for Political and Social Research.

Winkler, R., C. Cheng, and S. Golding. 2011. "Boom or bust? How migration impacts population composition in different types of natural resource dependent communities in the rural US." In L. Kulcsar and K. Curtis (eds.), International Handbook of Rural Demography. The Springer Series on Demographic Methods and Population Analysis. New York: Springer. 


\section{Appendix 1. Variable Naming Conventions}

The first letter indicates what type of data the variable holds:

"b" - births

"p" - starting population (adjusted), observed at Census 2000

"e" - 2010 population absent migration, or in other words the expected population in 2010

"f" - final population (adjusted), observed at Census 2010

"m" - net Migrants (NM= expected population 2010 - final population 2010)

" $r$ " - net migration rate (NMR= net Migrants/expected population in 2010). If expected population is zero, then NMR is missing.

The second character is a number, representing the decade for which the data refer. In this file, all the variables refer to the time period 2000-2010 and are represented by " 0 ". The purpose of including this character is to ease comparison to data files with net migration estimates in prior decades.

The following set of letters refers to the population sub-group by race, ethnicity, and sex. The third character indicates race.

"b" - non-Hispanic Blacks

"w" - non-Hispanic Whites

"o" - non-Hispanics of some other race (Asian, American Indian, Native Hawaiian, etc.)

" $t$ " - total of all races combined

The fourth character refers to Hispanic origin.

"h" - Hispanic

"n" - not Hispanic

" $\mathrm{t}$ " - total of Hispanic and not

The fifth character indicates sex:

"m" - male

"f" - female

" $t$ " - total of both sexes

The sixth and seventh characters indicate the cohort's five year age group:

"0" - ages 0-4

"5" - ages 5-9

"10" - ages 10-14

$\cdots$

" $80 "$ - ages $80-84$

" 85 " - ages 85 and over

For the final population in 2010, expected population in 2010, net migration estimates and net migration rates, the two age slot describes that cohort's age in the year 2010. For births, the 
characters describe the age group these people would age into in 2010. For instance, those born 2000-2004 are labeled as b0xxx5 because in 2010 they would be age 5-9, whereas those born 2005-2009 are labeled as b0xxx0. For the 2000 population the age slot describes that cohort's age in the year 2000 . 


\section{Appendix 2. Adjustments to Census 2000 and Census 2010}

Census 2000 Adjustments based on ACE Revision II and Demographic Analysis (under age 10). Positive values indicate a net undercount was estimated and our team increased census enumerated populations. Negative values indicate a net overcount was estimated and our team decreased census enumerated populations. All values are in percent. Black values were applied to Black and Hispanic populations. Nonblack values were applied to non-Hispanic White, AIAN, and Asian populations.

\begin{tabular}{|l|r|r|r|r|}
\hline \multicolumn{7}{|c|}{ Percent Adjustment to Census 2000 } \\
\hline \multicolumn{1}{|c|}{ Age } & \multicolumn{1}{c|}{ Black Male } & \multicolumn{1}{c|}{ Black Female } & Nonblack male & Nonblack female \\
\hline 0 to 4 & 5.3 & 5.4 & 3.3 & 3.8 \\
\hline 5 to 9 & 1.4 & 1.9 & 1.1 & 1.5 \\
\hline 10 to 17 & -0.59 & -0.55 & -1.46 & -1.44 \\
\hline 18 to 29 & 0.04 & 0 & 0.17 & -1.54 \\
\hline 30 to 49 & 0.11 & -0.40 & -0.48 & -0.63 \\
\hline $50+$ & -2.54 & -2.51 & -2.15 & -2.42 \\
\hline
\end{tabular}


Census 2010 Adjustments based on Demographic Analysis (DA) and Census Coverage Measurement survey (CCM). Positive values indicate a net undercount was estimated and our team increased census enumerated populations. Negative values indicate a net overcount was estimated and our team decreased census enumerated populations. All values are in percent.

\begin{tabular}{|c|c|c|c|c|c|c|c|c|c|c|c|c|}
\hline \multicolumn{13}{|c|}{ Percent Adjustment to Census 2010} \\
\hline \multirow[t]{2}{*}{ Age } & \multicolumn{2}{|c|}{ NH White } & \multicolumn{2}{|c|}{ Black } & \multicolumn{2}{|c|}{ Hispanic } & \multicolumn{2}{|c|}{ AIAN } & \multicolumn{2}{|c|}{ Asian } & \multicolumn{2}{|c|}{ NHPI } \\
\hline & $\mathrm{M}$ & $\mathrm{F}$ & $\mathrm{M}$ & $\mathrm{F}$ & $\mathrm{M}$ & $\mathrm{F}$ & $\mathrm{M}$ & $\mathrm{F}$ & $\mathrm{M}$ & $\mathrm{F}$ & $\mathrm{M}$ & $\mathrm{F}$ \\
\hline $0-4$ & 2.6 & 2.6 & 4.4 & 4.1 & 7.5 & 7.5 & 6.6 & 6.2 & 0.0 & 0.0 & 0.0 & 0.0 \\
\hline $5-9$ & 0.8 & 0.7 & 0.9 & 0.7 & 2.2 & 2.2 & 1.4 & 1.1 & 0.0 & 0.0 & 0.0 & 0.0 \\
\hline $10-14$ & 0.0 & 0.0 & 0.0 & 0.0 & 0.0 & 0.0 & 0.0 & 0.0 & 0.0 & 0.0 & 0.0 & 0.0 \\
\hline $15-19$ & -2.0 & -1.5 & -1.1 & -1.0 & 0.0 & 0.0 & -2.0 & -1.5 & 0.0 & 0.0 & 0.0 & 0.0 \\
\hline $20-24$ & 0.0 & 0.0 & 4.1 & 0.0 & 2.7 & 0.0 & 4.1 & 2.0 & 0.0 & 0.0 & 0.0 & 0.0 \\
\hline $25-29$ & 0.0 & 0.0 & 9.0 & 0.0 & 6.0 & 2.2 & 9.0 & 4.5 & 0.0 & 0.0 & 0.0 & 0.0 \\
\hline $30-34$ & 1.5 & -1.2 & 6.6 & -1.1 & 4.4 & 1.6 & 6.6 & 3.3 & 0.0 & 0.0 & 0.0 & 0.0 \\
\hline $35-39$ & 1.1 & -1.6 & 6.8 & -1.3 & 4.5 & 1.7 & 6.8 & 3.4 & 0.0 & 0.0 & 0.0 & 0.0 \\
\hline $40-44$ & 0.0 & -1.5 & 5.9 & -1.1 & 4.0 & 1.5 & 5.9 & 3.0 & 0.0 & 0.0 & 0.0 & 0.0 \\
\hline $45-49$ & 0.0 & -1.5 & 6.5 & -0.6 & 4.3 & 1.6 & 6.5 & 3.3 & 0.0 & 0.0 & 0.0 & 0.0 \\
\hline $50-54$ & 0.0 & -2.2 & 4.8 & -0.9 & 3.2 & 1.2 & 4.8 & 2.4 & 0.0 & 0.0 & 0.0 & 0.0 \\
\hline $55-59$ & 0.0 & -2.5 & 2.8 & -1.0 & 1.9 & 0.7 & 2.8 & 1.4 & 0.0 & 0.0 & 0.0 & 0.0 \\
\hline $60-64$ & 0.0 & -3.3 & 0.0 & -1.2 & 0.0 & 0.0 & 0.0 & 0.0 & 0.0 & 0.0 & 0.0 & 0.0 \\
\hline $65-69$ & 0.0 & -2.7 & 1.8 & -1.3 & 0.0 & 0.0 & 1.8 & 0.9 & 0.0 & 0.0 & 0.0 & 0.0 \\
\hline $70-74$ & 0.0 & -1.3 & 2.8 & -0.9 & 0.0 & 0.0 & 2.8 & 1.4 & 0.0 & 0.0 & 0.0 & 0.0 \\
\hline $75-79$ & 0.0 & -1.5 & -1.6 & -1.1 & 0.0 & 0.0 & -1.6 & -0.8 & 0.0 & 0.0 & 0.0 & 0.0 \\
\hline $80-84$ & 0.0 & -1.6 & -1.6 & -1.1 & 0.0 & 0.0 & -1.6 & -0.8 & 0.0 & 0.0 & 0.0 & 0.0 \\
\hline $85+$ & 0.0 & 0.0 & 2.3 & 3.1 & 0.0 & 0.0 & 2.3 & 1.2 & 0.0 & 0.0 & 0.0 & 0.0 \\
\hline
\end{tabular}




\section{Appendix 3. Broomfield County, Colorado}

Broomfield County, Colorado was created from parts of Adams, Boulder, Jefferson, and Weld Counties in 2001. The US Census Population Estimates base for 4/1/2000 includes Broomfield County and adjusts the population of Adams, Boulder, Jefferson, and Weld counties accordingly, even though Broomfield was not created until 2001. So, we have a consistent starting (Census 2000) and final (Census 2010) population for these counties, using the Population Estimates base. Birth and death data, however, from the National Center for Health Statistics do not delineate the current county geographies until 2003. This means that we must estimate the number of births and deaths in each of these respective counties for 2000, 2001, and 2002.

To create death estimates, we began by calculating the percent of the population of each of the original counties that was moved into Broomfield County by age, sex, race, and Hispanic origin. We did this using the Intercensal Population Estimates file and calculating the percent difference between the census enumerated population (which does not reflect Broomfield County's creation) and the population estimates base (which is adjusted to distribute the population of Adams, Boulder, Jefferson, and Weld Counties to the newly created Broomfield appropriately). We then multiplied these proportions times the observed deaths in the original counties in 2000, 2001, and 2002 to estimate the number of deaths that needed to be subtracted from each of these counties and given to Broomfield County.

Birth estimates were created in a similar fashion excect that instead of relying on the proportion of the population that needed to be moved for each age group, we focused only on the female population in prime childbearing ages (age 15-44). We multiplied the proportion of the female childbearing population who were moved out of each of the original counties times the observed births in those counties in 2000, 2001, and 2002 to determine the number of births to be removed from each of the original counties and added to Broomfield County.

Please note that because of these added complexities and assumptions, net migration estimates for Broomfield, Adams, Boulder, Jefferson, and Weld counties (2000-2010) should be treated with some caution. 


\section{Appendix 4. County Boundary Changes and FIPS codes}

Between 2000 and 2010, some counties and census areas in Alaska and Virginia changed boundaries and/or naming conventions. In order to create consistent boundaries and geographic reference for the Net Migration Estimates 2000-2010, we created the following county groupings and use the associated NME Fips codes and NME Name in the Net Migration Estimates data files.

\begin{tabular}{|c|c|c|c|c|c|}
\hline State & 2000 County Name & $\begin{array}{r}2000 \\
\text { Fips }\end{array}$ & $\begin{array}{l}2010 \\
\text { Fips }\end{array}$ & $\begin{array}{c}\text { NME } \\
\text { Fips }\end{array}$ & NME Name \\
\hline Alaska & Denali Borough & & 068 & 991 & Denali Yukon-Koyukuk Southeast Fairbanks \\
\hline Alaska & Yukon-Koyukuk Census Area & 290 & 290 & 991 & Denali Yukon-Koyukuk Southeast Fairbanks \\
\hline Alaska & Southeast Fairbanks Census Area & 240 & 240 & 991 & Denali Yukon-Koyukuk Southeast Fairbanks \\
\hline Alaska & Prince of Wales-Outer Ketchikan Census Area & 201 & - & 992 & Wrangell Petersburg Prince of Wales Ketchikan \\
\hline Alaska & Wrangell-Petersburg Census Area & 280 & - & 992 & Wrangell Petersburg Prince of Wales Ketchikan \\
\hline Alaska & Ketchikan Census Area & 130 & 130 & 992 & Wrangell Petersburg Prince of Wales Ketchikan \\
\hline Alaska & Wrangell City and Borough & & 275 & 992 & Wrangell Petersburg Prince of Wales Ketchikan \\
\hline Alaska & Petersburg Census Area & & 195 & 992 & Wrangell Petersburg Prince of Wales Ketchikan \\
\hline Alaska & Prince of Wales-Hyder Census Area & & 198 & 992 & Wrangell Petersburg Prince of Wales Ketchikan \\
\hline Alaska & Skagway Municipality & & 230 & 993 & Skagway Hoonah-Angoon Yakutat-Angoon \\
\hline Alaska & Hoonah-Angoon Census Area & & 105 & 993 & Skagway Hoonah-Angoon Yakutat-Angoon \\
\hline Alaska & Yakutat-Angoon Census Area & - & - & 993 & Skagway Hoonah-Angoon Yakutat-Angoon \\
\hline Virginia & Clifton Forge Independent City & 560 & - & 005 & Alleghany County \\
\hline Virginia & Alleghany & 005 & 005 & 005 & Alleghany County \\
\hline
\end{tabular}




\section{Appendix 5. Adjustments to Birth Estimates, 2009}

The following tables list the counties where adjustments to birth estimates 2009 were necessary by race/ethnicity.

\section{Non-Hispanic White}

\begin{tabular}{|c|c|c|}
\hline StCo Fips & State & County Name \\
\hline 1001 & Alabama & Autauga County \\
\hline 1007 & Alabama & Bibb County \\
\hline 1029 & Alabama & Cleburne County \\
\hline 1053 & Alabama & Escambia County \\
\hline 1091 & Alabama & Marengo County \\
\hline 1113 & Alabama & Russell County \\
\hline 2070 & Alaska & Dillingham Census Area \\
\hline 2100 & Alaska & Haines Borough \\
\hline 4009 & Arizona & Graham County \\
\hline 5023 & Arkansas & Cleburne County \\
\hline 5067 & Arkansas & Jackson County \\
\hline 5095 & Arkansas & Monroe County \\
\hline 5107 & Arkansas & Phillips County \\
\hline 6105 & California & Trinity County \\
\hline 8015 & Colorado & Chaffee County \\
\hline 8063 & Colorado & Kit Carson County \\
\hline 8091 & Colorado & Ouray County \\
\hline 12015 & Florida & Charlotte County \\
\hline 12129 & Florida & Wakulla County \\
\hline 12131 & Florida & Walton County \\
\hline 13003 & Georgia & Atkinson County \\
\hline 13025 & Georgia & Brantley County \\
\hline 13053 & Georgia & Chattahoochee County \\
\hline 13079 & Georgia & Crawford County \\
\hline 13105 & Georgia & Elbert County \\
\hline 13167 & Georgia & Johnson County \\
\hline 13179 & Georgia & Liberty County \\
\hline 13209 & Georgia & Montgomery County \\
\hline 13249 & Georgia & Schley County \\
\hline 13261 & Georgia & Sumter County \\
\hline 13291 & Georgia & Union County \\
\hline 13297 & Georgia & Walton County \\
\hline 13309 & Georgia & Wheeler County \\
\hline 13315 & Georgia & Wilcox County \\
\hline 16013 & Idaho & Blaine County \\
\hline 16021 & Idaho & Boundary County \\
\hline 16071 & Idaho & Oneida County \\
\hline
\end{tabular}




\begin{tabular}{|c|c|c|}
\hline 16073 & Idaho & Owyhee County \\
\hline 17065 & Illinois & Hamilton County \\
\hline 18047 & Indiana & Franklin County \\
\hline 18111 & Indiana & Newton County \\
\hline 18129 & Indiana & Posey County \\
\hline 19009 & lowa & Audubon County \\
\hline 19053 & lowa & Decatur County \\
\hline 19073 & lowa & Greene County \\
\hline 19083 & lowa & Hardin County \\
\hline 19111 & lowa & Lee County \\
\hline 19151 & lowa & Pocahontas County \\
\hline 20007 & Kansas & Barber County \\
\hline 20039 & Kansas & Decatur County \\
\hline 20081 & Kansas & Haskell County \\
\hline 20113 & Kansas & McPherson County \\
\hline 20121 & Kansas & Miami County \\
\hline 20143 & Kansas & Ottawa County \\
\hline 21001 & Kentucky & Adair County \\
\hline 21005 & Kentucky & Anderson County \\
\hline 21017 & Kentucky & Bourbon County \\
\hline 21035 & Kentucky & Calloway County \\
\hline 21041 & Kentucky & Carroll County \\
\hline 21047 & Kentucky & Christian County \\
\hline 21115 & Kentucky & Johnson County \\
\hline 21149 & Kentucky & McLean County \\
\hline 21161 & Kentucky & Mason County \\
\hline 21165 & Kentucky & Menifee County \\
\hline 21167 & Kentucky & Mercer County \\
\hline 21239 & Kentucky & Woodford County \\
\hline 22115 & Louisiana & Vernon Parish \\
\hline 23003 & Maine & Aroostook County \\
\hline 23023 & Maine & Sagadahoc County \\
\hline 24019 & Maryland & Dorchester County \\
\hline 26097 & Michigan & Mackinac County \\
\hline 26131 & Michigan & Ontonagon County \\
\hline 26141 & Michigan & Presque Isle County \\
\hline 27031 & Minnesota & Cook County \\
\hline 27077 & Minnesota & Lake of the Woods County \\
\hline 27161 & Minnesota & Waseca County \\
\hline 28131 & Mississippi & Stone County \\
\hline 29003 & Missouri & Andrew County \\
\hline 29023 & Missouri & Butler County \\
\hline 29067 & Missouri & Douglas County \\
\hline 29125 & Missouri & Maries County \\
\hline 29163 & Missouri & Pike County \\
\hline
\end{tabular}




\begin{tabular}{|c|c|c|}
\hline 29185 & Missouri & St. Clair County \\
\hline 30023 & Montana & Deer Lodge County \\
\hline 30033 & Montana & Garfield County \\
\hline 30045 & Montana & Judith Basin County \\
\hline 30055 & Montana & McCone County \\
\hline 30057 & Montana & Madison County \\
\hline 30061 & Montana & Mineral County \\
\hline 31017 & Nebraska & Brown County \\
\hline 31045 & Nebraska & Dawes County \\
\hline 31121 & Nebraska & Merrick County \\
\hline 31165 & Nebraska & Sioux County \\
\hline 35011 & New Mexico & De Baca County \\
\hline 35021 & New Mexico & Harding County \\
\hline 36045 & New York & Jefferson County \\
\hline 36095 & New York & Schoharie County \\
\hline 36101 & New York & Steuben County \\
\hline 38031 & North Dakota & Foster County \\
\hline 38069 & North Dakota & Pierce County \\
\hline 39053 & Ohio & Gallia County \\
\hline 39127 & Ohio & Perry County \\
\hline 40029 & Oklahoma & Coal County \\
\hline 40055 & Oklahoma & Greer County \\
\hline 40059 & Oklahoma & Harper County \\
\hline 40077 & Oklahoma & Latimer County \\
\hline 45003 & South Carolina & Aiken County \\
\hline 48041 & Texas & Brazos County \\
\hline 55141 & Wisconsin & Wood County \\
\hline 55017 & Wisconsin & Chippewa County \\
\hline 48099 & Texas & Coryell County \\
\hline 42015 & Pennsylvania & Bradford County \\
\hline 47043 & Tennessee & Dickson County \\
\hline 55097 & Wisconsin & Portage County \\
\hline 40147 & Oklahoma & Washington County \\
\hline 54099 & West Virginia & Wayne County \\
\hline 47013 & Tennessee & Campbell County \\
\hline 42117 & Pennsylvania & Tioga County \\
\hline 50025 & Vermont & Windham County \\
\hline 54035 & West Virginia & Jackson County \\
\hline 53065 & Washington & Stevens County \\
\hline 50017 & Vermont & Orange County \\
\hline 47109 & Tennessee & McNairy County \\
\hline 51009 & Virginia & Amherst County \\
\hline 54047 & West Virginia & McDowell County \\
\hline 40153 & Oklahoma & Woodward County \\
\hline 47079 & Tennessee & Henry County \\
\hline
\end{tabular}




\begin{tabular}{|c|c|c|}
\hline 41045 & Oregon & Malheur County \\
\hline 47159 & Tennessee & Smith County \\
\hline 48477 & Texas & Washington County \\
\hline 46035 & South Dakota & Davison County \\
\hline 48015 & Texas & Austin County \\
\hline 51840 & Virginia & Winchester city \\
\hline 42093 & Pennsylvania & Montour County \\
\hline 47101 & Tennessee & Lewis County \\
\hline 55137 & Wisconsin & Waushara County \\
\hline 47085 & Tennessee & Humphreys County \\
\hline 41031 & Oregon & Jefferson County \\
\hline 51037 & Virginia & Charlotte County \\
\hline 56019 & Wyoming & Johnson County \\
\hline 46079 & South Dakota & Lake County \\
\hline 46115 & South Dakota & Spink County \\
\hline 46067 & South Dakota & Hutchinson County \\
\hline 47087 & Tennessee & Jackson County \\
\hline 48193 & Texas & Hamilton County \\
\hline 51530 & Virginia & Buena Vista city \\
\hline 46077 & South Dakota & Kingsbury County \\
\hline 47175 & Tennessee & Van Buren County \\
\hline 41063 & Oregon & Wallowa County \\
\hline 55091 & Wisconsin & Pepin County \\
\hline 53055 & Washington & San Juan County \\
\hline 54095 & West Virginia & Tyler County \\
\hline 48369 & Texas & Parmer County \\
\hline 48317 & Texas & Martin County \\
\hline 46105 & South Dakota & Perkins County \\
\hline 45005 & South Carolina & Allendale County \\
\hline 56027 & Wyoming & Niobrara County \\
\hline 53023 & Washington & Garfield County \\
\hline 48101 & Texas & Cottle County \\
\hline 46119 & South Dakota & $\begin{array}{l}\text { Sully County } \\
\text { Denali Yukon-Koyukuk Southeas }\end{array}$ \\
\hline 2991 & Alaska & $\begin{array}{l}\text { Fairbanks Comp } \\
\text { Skagway Hoonah-Angoon Yakutat- }\end{array}$ \\
\hline 2993 & Alaska & Angoon Comp \\
\hline 8121 & Colorado & Washington County \\
\hline 12123 & Florida & Taylor County \\
\hline 13035 & Georgia & Butts County \\
\hline 13177 & Georgia & Lee County \\
\hline 16085 & Idaho & Valley County \\
\hline 17005 & Illinois & Bond County \\
\hline 17023 & Illinois & Clark County \\
\hline 18165 & Indiana & Vermillion County \\
\hline
\end{tabular}




\begin{tabular}{|c|c|c|}
\hline 19007 & lowa & Appanoose County \\
\hline 27167 & Minnesota & Wilkin County \\
\hline 35047 & New Mexico & San Miguel County \\
\hline 1027 & Alabama & Clay County \\
\hline 2016 & Alaska & Aleutians West Census Area \\
\hline 2185 & Alaska & North Slope Borough \\
\hline 2270 & Alaska & Wade Hampton Census Area \\
\hline 4012 & Arizona & La Paz County \\
\hline 5017 & Arkansas & Chicot County \\
\hline 5021 & Arkansas & Clay County \\
\hline 5039 & Arkansas & Dallas County \\
\hline 5081 & Arkansas & Little River County \\
\hline 5143 & Arkansas & Washington County \\
\hline 6049 & California & Modoc County \\
\hline 8019 & Colorado & Clear Creek County \\
\hline 8037 & Colorado & Eagle County \\
\hline 8039 & Colorado & Elbert County \\
\hline 8043 & Colorado & Fremont County \\
\hline 8047 & Colorado & Gilpin County \\
\hline 8093 & Colorado & Park County \\
\hline 8111 & Colorado & San Juan County \\
\hline 8113 & Colorado & San Miguel County \\
\hline 13011 & Georgia & Banks County \\
\hline 13065 & Georgia & Clinch County \\
\hline 13093 & Georgia & Dooly County \\
\hline 13133 & Georgia & Greene County \\
\hline 13193 & Georgia & Macon County \\
\hline 13229 & Georgia & Pierce County \\
\hline 13251 & Georgia & Screven County \\
\hline 13259 & Georgia & Stewart County \\
\hline 13265 & Georgia & Taliaferro County \\
\hline 13267 & Georgia & Tattnall County \\
\hline 13301 & Georgia & Warren County \\
\hline 13319 & Georgia & Wilkinson County \\
\hline 16017 & Idaho & Bonner County \\
\hline 16035 & Idaho & Clearwater County \\
\hline 16063 & Idaho & Lincoln County \\
\hline 17009 & Illinois & Brown County \\
\hline 17013 & Illinois & Calhoun County \\
\hline 17079 & Illinois & Jasper County \\
\hline 17121 & Illinois & Marion County \\
\hline 17173 & Illinois & Shelby County \\
\hline 17189 & Illinois & Washington County \\
\hline 17191 & Illinois & Wayne County \\
\hline 18085 & Indiana & Kosciusko County \\
\hline
\end{tabular}




\begin{tabular}{|c|c|c|}
\hline 18115 & Indiana & Ohio County \\
\hline 18169 & Indiana & Wabash County \\
\hline 18179 & Indiana & Wells County \\
\hline 19147 & lowa & Palo Alto County \\
\hline 19173 & lowa & Taylor County \\
\hline 19183 & lowa & Washington County \\
\hline 19189 & lowa & Winnebago County \\
\hline 20001 & Kansas & Allen County \\
\hline 20013 & Kansas & Brown County \\
\hline 20021 & Kansas & Cherokee County \\
\hline 20053 & Kansas & Ellsworth County \\
\hline 20057 & Kansas & Ford County \\
\hline 20067 & Kansas & Grant County \\
\hline 20095 & Kansas & Kingman County \\
\hline 20115 & Kansas & Marion County \\
\hline 20199 & Kansas & Wallace County \\
\hline 20205 & Kansas & Wilson County \\
\hline 21075 & Kentucky & Fulton County \\
\hline 21077 & Kentucky & Gallatin County \\
\hline 21089 & Kentucky & Greenup County \\
\hline 21095 & Kentucky & Harlan County \\
\hline 21099 & Kentucky & Hart County \\
\hline 21159 & Kentucky & Martin County \\
\hline 21217 & Kentucky & Taylor County \\
\hline 22023 & Louisiana & Cameron Parish \\
\hline 22029 & Louisiana & Concordia Parish \\
\hline 22063 & Louisiana & Livingston Parish \\
\hline 22075 & Louisiana & Plaquemines Parish \\
\hline 22077 & Louisiana & Pointe Coupee Parish \\
\hline 22083 & Louisiana & Richland Parish \\
\hline 22125 & Louisiana & West Feliciana Parish \\
\hline 24029 & Maryland & Kent County \\
\hline 26001 & Michigan & Alcona County \\
\hline 26003 & Michigan & Alger County \\
\hline 26135 & Michigan & Oscoda County \\
\hline 26153 & Michigan & Schoolcraft County \\
\hline 26165 & Michigan & Wexford County \\
\hline 27007 & Minnesota & Beltrami County \\
\hline 27087 & Minnesota & Mahnomen County \\
\hline 27091 & Minnesota & Martin County \\
\hline 27099 & Minnesota & Mower County \\
\hline 27107 & Minnesota & Norman County \\
\hline 27119 & Minnesota & Polk County \\
\hline 27151 & Minnesota & Swift County \\
\hline 27155 & Minnesota & Traverse County \\
\hline
\end{tabular}




\begin{tabular}{|c|c|c|}
\hline 27165 & Minnesota & Watonwan County \\
\hline 28019 & Mississippi & Choctaw County \\
\hline 28037 & Mississippi & Franklin County \\
\hline 28045 & Mississippi & Hancock County \\
\hline 28079 & Mississippi & Leake County \\
\hline 28091 & Mississippi & Marion County \\
\hline 28101 & Mississippi & Newton County \\
\hline 28105 & Mississippi & Oktibbeha County \\
\hline 28109 & Mississippi & Pearl River County \\
\hline 28119 & Mississippi & Quitman County \\
\hline 28151 & Mississippi & Washington County \\
\hline 28157 & Mississippi & Wilkinson County \\
\hline 29041 & Missouri & Chariton County \\
\hline 29053 & Missouri & Cooper County \\
\hline 29063 & Missouri & DeKalb County \\
\hline 29073 & Missouri & Gasconade County \\
\hline 29111 & Missouri & Lewis County \\
\hline 29127 & Missouri & Marion County \\
\hline 29153 & Missouri & Ozark County \\
\hline 29209 & Missouri & Stone County \\
\hline 30035 & Montana & Glacier County \\
\hline 30041 & Montana & Hill County \\
\hline 30043 & Montana & Jefferson County \\
\hline 30073 & Montana & Pondera County \\
\hline 31027 & Nebraska & Cedar County \\
\hline 31029 & Nebraska & Chase County \\
\hline 31059 & Nebraska & Fillmore County \\
\hline 31061 & Nebraska & Franklin County \\
\hline 31063 & Nebraska & Frontier County \\
\hline 31071 & Nebraska & Garfield County \\
\hline 31081 & Nebraska & Hamilton County \\
\hline 31083 & Nebraska & Harlan County \\
\hline 31107 & Nebraska & Knox County \\
\hline 31113 & Nebraska & Logan County \\
\hline 31133 & Nebraska & Pawnee County \\
\hline 31137 & Nebraska & Phelps County \\
\hline 31161 & Nebraska & Sheridan County \\
\hline 31163 & Nebraska & Sherman County \\
\hline 31171 & Nebraska & Thomas County \\
\hline 31181 & Nebraska & Webster County \\
\hline 32015 & Nevada & Lander County \\
\hline 35017 & New Mexico & Grant County \\
\hline 35019 & New Mexico & Guadalupe County \\
\hline 35023 & New Mexico & Hidalgo County \\
\hline 35033 & New Mexico & Mora County \\
\hline
\end{tabular}




\begin{tabular}{|c|c|c|}
\hline 35053 & New Mexico & Socorro County \\
\hline 37117 & North Carolina & Martin County \\
\hline 37189 & North Carolina & Watauga County \\
\hline 38001 & North Dakota & Adams County \\
\hline 38073 & North Dakota & Ransom County \\
\hline 38081 & North Dakota & Sargent County \\
\hline 38091 & North Dakota & Steele County \\
\hline 38095 & North Dakota & Towner County \\
\hline 39067 & Ohio & Harrison County \\
\hline 39111 & Ohio & Monroe County \\
\hline 39121 & Ohio & Noble County \\
\hline 40001 & Oklahoma & Adair County \\
\hline 40009 & Oklahoma & Beckham County \\
\hline 40091 & Oklahoma & McIntosh County \\
\hline 40103 & Oklahoma & Noble County \\
\hline 40105 & Oklahoma & Nowata County \\
\hline 40113 & Oklahoma & Osage County \\
\hline 40115 & Oklahoma & Ottawa County \\
\hline 40129 & Oklahoma & Roger Mills County \\
\hline 41037 & Oregon & Lake County \\
\hline 41049 & Oregon & Morrow County \\
\hline 42031 & Pennsylvania & Clarion County \\
\hline 42047 & Pennsylvania & Elk County \\
\hline 42131 & Pennsylvania & Wyoming County \\
\hline 45037 & South Carolina & Edgefield County \\
\hline 45039 & South Carolina & Fairfield County \\
\hline 45053 & South Carolina & Jasper County \\
\hline 45065 & South Carolina & McCormick County \\
\hline 45069 & South Carolina & Marlboro County \\
\hline 46007 & South Dakota & Bennett County \\
\hline 46069 & South Dakota & Hyde County \\
\hline 46087 & South Dakota & McCook County \\
\hline 46091 & South Dakota & Marshall County \\
\hline 46093 & South Dakota & Meade County \\
\hline 46123 & South Dakota & Tripp County \\
\hline 47003 & Tennessee & Bedford County \\
\hline 47095 & Tennessee & Lake County \\
\hline 47121 & Tennessee & Meigs County \\
\hline 47125 & Tennessee & Montgomery County \\
\hline 48003 & Texas & Andrews County \\
\hline 48009 & Texas & Archer County \\
\hline 48025 & Texas & Bee County \\
\hline 48069 & Texas & Castro County \\
\hline 48079 & Texas & Cochran County \\
\hline 48083 & Texas & Coleman County \\
\hline
\end{tabular}




\begin{tabular}{|c|c|c|}
\hline 48107 & Texas & Crosby County \\
\hline 48117 & Texas & Deaf Smith County \\
\hline 48151 & Texas & Fisher County \\
\hline 48169 & Texas & Garza County \\
\hline 48211 & Texas & Hemphill County \\
\hline 48225 & Texas & Houston County \\
\hline 48237 & Texas & Jack County \\
\hline 48249 & Texas & Jim Wells County \\
\hline 48271 & Texas & Kinney County \\
\hline 48281 & Texas & Lampasas County \\
\hline 48315 & Texas & Marion County \\
\hline 48325 & Texas & Medina County \\
\hline 48327 & Texas & Menard County \\
\hline 48333 & Texas & Mills County \\
\hline 48359 & Texas & Oldham County \\
\hline 48377 & Texas & Presidio County \\
\hline 48401 & Texas & Rusk County \\
\hline 48405 & Texas & San Augustine County \\
\hline 48425 & Texas & Somervell County \\
\hline 48429 & Texas & Stephens County \\
\hline 48433 & Texas & Stonewall County \\
\hline 48435 & Texas & Sutton County \\
\hline 48461 & Texas & Upton County \\
\hline 48475 & Texas & Ward County \\
\hline 48503 & Texas & Young County \\
\hline 49055 & Utah & Wayne County \\
\hline 51036 & Virginia & Charles City County \\
\hline 51135 & Virginia & Nottoway County \\
\hline 51520 & Virginia & Bristol city \\
\hline 51600 & Virginia & Fairfax city \\
\hline 51683 & Virginia & Manassas city \\
\hline 51735 & Virginia & Poquoson city \\
\hline 51830 & Virginia & Williamsburg city \\
\hline 53069 & Washington & Wahkiakum County \\
\hline 54005 & West Virginia & Boone County \\
\hline 54021 & West Virginia & Gilmer County \\
\hline 54029 & West Virginia & Hancock County \\
\hline 54105 & West Virginia & Wirt County \\
\hline 55078 & Wisconsin & Menominee County \\
\hline 55125 & Wisconsin & Vilas County \\
\hline 56029 & Wyoming & Park County \\
\hline 56043 & Wyoming & Washakie County \\
\hline
\end{tabular}




\section{American Indian/Alaska Native (AIAN)}

\begin{tabular}{|c|c|c|}
\hline StCo Fips & State & County Name \\
\hline 2016 & Alaska & Aleutians West Census Area \\
\hline 2070 & Alaska & Dillingham Census Area \\
\hline 2185 & Alaska & North Slope Borough \\
\hline 2270 & Alaska & Wade Hampton Census Area \\
\hline 2991 & Alaska & Denali Yukon-Koyukuk Southeast Fairbanks \\
\hline 4009 & Arizona & Graham County \\
\hline 5081 & Arkansas & Little River County \\
\hline 6049 & California & Modoc County \\
\hline 8043 & Colorado & Fremont County \\
\hline 16035 & Idaho & Clearwater County \\
\hline 17191 & Illinois & Wayne County \\
\hline 22063 & Louisiana & Livingston Parish \\
\hline 23003 & Maine & Aroostook County \\
\hline 26153 & Michigan & Schoolcraft County \\
\hline 27087 & Minnesota & Mahnomen County \\
\hline 27107 & Minnesota & Norman County \\
\hline 30035 & Montana & Glacier County \\
\hline 30041 & Montana & Hill County \\
\hline 30073 & Montana & Pondera County \\
\hline 31045 & Nebraska & Dawes County \\
\hline 31107 & Nebraska & Knox County \\
\hline 32015 & Nevada & Lander County \\
\hline 36045 & New York & Jefferson County \\
\hline 40105 & Oklahoma & Nowata County \\
\hline 40113 & Oklahoma & Osage County \\
\hline 40129 & Oklahoma & Roger Mills County \\
\hline 40147 & Oklahoma & Washington County \\
\hline 41031 & Oregon & Jefferson County \\
\hline 41063 & Oregon & Wallowa County \\
\hline 46007 & South Dakota & Bennett County \\
\hline 48041 & Texas & Brazos County \\
\hline 53065 & Washington & Stevens County \\
\hline 55078 & Wisconsin & Menominee County \\
\hline 55141 & Wisconsin & Wood County \\
\hline
\end{tabular}

Black

\begin{tabular}{lcl} 
StCo Fips & State Name & County Name \\
\hline 5081 & Arkansas & Little River County \\
12015 & Florida & Charlotte County \\
12123 & Florida & Taylor County
\end{tabular}




\begin{tabular}{|c|c|c|}
\hline 13025 & Georgia & Brantley County \\
\hline 13053 & Georgia & Chattahoochee County \\
\hline 13065 & Georgia & Clinch County \\
\hline 13093 & Georgia & Dooly County \\
\hline 13105 & Georgia & Elbert County \\
\hline 13167 & Georgia & Johnson County \\
\hline 13177 & Georgia & Lee County \\
\hline 13179 & Georgia & Liberty County \\
\hline 13193 & Georgia & Macon County \\
\hline 13251 & Georgia & Screven County \\
\hline 13261 & Georgia & Sumter County \\
\hline 13301 & Georgia & Warren County \\
\hline 13309 & Georgia & Wheeler County \\
\hline 13319 & Georgia & Wilkinson County \\
\hline 21017 & Kentucky & Bourbon County \\
\hline 21047 & Kentucky & Christian County \\
\hline 22077 & Louisiana & Pointe Coupee Parish \\
\hline 22083 & Louisiana & Richland Parish \\
\hline 23003 & Maine & Aroostook County \\
\hline 24019 & Maryland & Dorchester County \\
\hline 24029 & Maryland & Kent County \\
\hline 27007 & Minnesota & Beltrami County \\
\hline 27119 & Minnesota & Polk County \\
\hline 28037 & Mississippi & Franklin County \\
\hline 28079 & Mississippi & Leake County \\
\hline 28091 & Mississippi & Marion County \\
\hline 28101 & Mississippi & Newton County \\
\hline 28105 & Mississippi & Oktibbeha County \\
\hline 28109 & Mississippi & Pearl River County \\
\hline 40113 & Oklahoma & Osage County \\
\hline 40147 & Oklahoma & Washington County \\
\hline 41045 & Oregon & Malheur County \\
\hline 47003 & Tennessee & Bedford County \\
\hline 47043 & Tennessee & Dickson County \\
\hline 48225 & Texas & Houston County \\
\hline 48475 & Texas & Ward County \\
\hline 50017 & Vermont & Orange County \\
\hline 51135 & Virginia & Nottoway County \\
\hline 51840 & Virginia & Winchester city \\
\hline 54029 & West Virginia & Hancock County \\
\hline
\end{tabular}




\begin{tabular}{lcl} 
Asian & & \\
StCo Fips & State Name & County Name \\
\hline 12015 & Florida & Charlotte County \\
13011 & Georgia & Banks County \\
17191 & Illinois & Wayne County \\
18085 & Indiana & Kosciusko County \\
18179 & Indiana & Wells County \\
21047 & Kentucky & Christian County \\
21167 & Kentucky & Mercer County \\
27099 & Minnesota & Mower County \\
27119 & Minnesota & Polk County \\
28151 & Mississippi & Washington County \\
31045 & Nebraska & Dawes County \\
36045 & New York & Jefferson County \\
40115 & Oklahoma & Ottawa County \\
40147 & Oklahoma & Washington County \\
48099 & Texas & Coryell County \\
48401 & Texas & Rusk County \\
51009 & Virginia & Amherst County \\
51520 & Virginia & Bristol city \\
51683 & Virginia & Manassas city \\
55017 & Wisconsin & Chippewa County \\
55097 & Wisconsin & Portage County \\
55141 & Wisconsin & Wood County \\
& &
\end{tabular}

\section{Hispanic}

\begin{tabular}{lcl} 
StCo Fips & State Name & County Name \\
\hline 12015 & Florida & Charlotte County \\
1001 & Alabama & Autauga County \\
5067 & Arkansas & Jackson County \\
5091 & Arkansas & Miller County \\
6049 & California & Modoc County \\
8037 & Colorado & Eagle County \\
13179 & Georgia & Liberty County \\
13193 & Georgia & Macon County \\
13197 & Georgia & Marion County \\
13209 & Georgia & Montgomery County \\
13229 & Georgia & Pierce County \\
13249 & Georgia & Schley County \\
13259 & Georgia & Stewart County \\
13261 & Georgia & Sumter County \\
13267 & Georgia & Tattnall County
\end{tabular}




\begin{tabular}{|c|c|c|}
\hline 13297 & Georgia & Walton County \\
\hline 16017 & Idaho & Bonner County \\
\hline 20013 & Kansas & Brown County \\
\hline 20057 & Kansas & Ford County \\
\hline 21035 & Kentucky & Calloway County \\
\hline 21047 & Kentucky & Christian County \\
\hline 21167 & Kentucky & Mercer County \\
\hline 27099 & Minnesota & Mower County \\
\hline 27119 & Minnesota & Polk County \\
\hline 27165 & Minnesota & Watonwan County \\
\hline 28109 & Mississippi & Pearl River County \\
\hline 31029 & Nebraska & Chase County \\
\hline 35011 & New Mexico & De Baca County \\
\hline 35017 & New Mexico & Grant County \\
\hline 35019 & New Mexico & Guadalupe County \\
\hline 35023 & New Mexico & Hidalgo County \\
\hline 35053 & New Mexico & Socorro County \\
\hline 36045 & New York & Jefferson County \\
\hline 37189 & North Carolina & Watauga County \\
\hline 40059 & Oklahoma & Harper County \\
\hline 40147 & Oklahoma & Washington County \\
\hline 41037 & Oregon & Lake County \\
\hline 41049 & Oregon & Morrow County \\
\hline 47125 & Tennessee & Montgomery County \\
\hline 48069 & Texas & Castro County \\
\hline 48079 & Texas & Cochran County \\
\hline 48107 & Texas & Crosby County \\
\hline 48117 & Texas & Deaf Smith County \\
\hline 48151 & Texas & Fisher County \\
\hline 48169 & Texas & Garza County \\
\hline 48237 & Texas & Jack County \\
\hline 48249 & Texas & Jim Wells County \\
\hline 48271 & Texas & Kinney County \\
\hline 48317 & Texas & Martin County \\
\hline 48325 & Texas & Medina County \\
\hline 48377 & Texas & Presidio County \\
\hline 48425 & Texas & Somervell County \\
\hline 48475 & Texas & Ward County \\
\hline 48477 & Texas & Washington County \\
\hline 48503 & Texas & Young County \\
\hline 49041 & Utah & Sevier County \\
\hline 51600 & Virginia & Fairfax city \\
\hline 51683 & Virginia & Manassas city \\
\hline 51830 & Virginia & Williamsburg city \\
\hline 55017 & Wisconsin & Chippewa County \\
\hline
\end{tabular}




\section{Appendix 6 \\ Detailed Codebook for Electronic Readable Data File}

\begin{tabular}{|c|c|}
\hline Variable & \\
\hline fips3188 & Federal Information Processing Standard code, 5-digit state-county \\
\hline stname & State Name \\
\hline ctyname & County Name \\
\hline rObnfo & Estimated Net Migration Rate in 2000s, black non-hispanic female, ages 0-4 \\
\hline rObnf5 & Estimated Net Migration Rate in 2000s, black non-hispanic female, ages 5-9 \\
\hline rObnf10 & Estimated Net Migration Rate in 2000s, black non-hispanic female, ages $10-14$ \\
\hline rObnf15 & Estimated Net Migration Rate in 2000s, black non-hispanic female, ages $15-1 \mathrm{~S}$ \\
\hline rObnf20 & Estimated Net Migration Rate in 2000s, black non-hispanic female, ages $20-2$ \\
\hline rObnf25 & Estimated Net Migration Rate 2000s, black non-hispanic female, ages 25-29 \\
\hline rObnf30 & Estimated Net Migration Rate 2000s, black non-hispanic female, ages 30-34 \\
\hline rObnf35 & Estimated Net Migration Rate 2000s, black non-hispanic female, ages 35-39 \\
\hline rObnf40 & Estimated Net Migration Rate 2000s, black non-hispanic female, ages $40-44$ \\
\hline rObnf45 & Estimated Net Migration Rate 2000s, black non-hispanic female, ages 45-49 \\
\hline rObnf50 & Estimated Net Migration Rate 2000s, black non-hispanic female, ages 50-54 \\
\hline rObnf55 & Estimated Net Migration Rate 2000s, black non-hispanic female, ages 55-59 \\
\hline rObnf60 & Estimated Net Migration Rate 2000s, black non-hispanic female, ages $60-64$ \\
\hline rObnf65 & Estimated Net Migration Rate 2000s, black non-hispanic female, ages 65-69 \\
\hline rObnf70 & Estimated Net Migration Rate 2000s, black non-hispanic female, ages $70-74$ \\
\hline rObnf75 & Estimated Net Migration Rate 2000s, black non-hispanic female, ages 75-79 \\
\hline rObnf80 & Estimated Net Migration Rate 2000s, black non-hispanic female, ages $80-84$ \\
\hline r0bnf85 & Estimated Net Migration Rate 2000s, black non-hispanic female, ages 85+ \\
\hline rObnmo & Estimated Net Migration Rate 2000s, black non-hispanic male, ages 0-4 \\
\hline rObnm5 & Estimated Net Migration Rate 2000s, black non-hispanic male, ages 5-9 \\
\hline rObnm10 & Estimated Net Migration Rate 2000s, black non-hispanic male, ages 10-14 \\
\hline rObnm15 & Estimated Net Migration Rate 2000s, black non-hispanic male, ages 15-19 \\
\hline rObnm20 & Estimated Net Migration Rate 2000s, black non-hispanic male, ages 20-24 \\
\hline rObnm25 & Estimated Net Migration Rate 2000s, black non-hispanic male, ages 25-29 \\
\hline rObnm30 & Estimated Net Migration Rate 2000s, black non-hispanic male, ages 30-34 \\
\hline rObnm35 & Estimated Net Migration Rate 2000s, black non-hispanic male, ages 35-39 \\
\hline rObnm40 & Estimated Net Migration Rate 2000s, black non-hispanic male, ages 40-44 \\
\hline rObnm45 & Estimated Net Migration Rate 2000s, black non-hispanic male, ages 45-49 \\
\hline rObnm50 & Estimated Net Migration Rate 2000s, black non-hispanic male, ages 50-54 \\
\hline rObnm55 & Estimated Net Migration Rate 2000s, black non-hispanic male, ages 55-59 \\
\hline rObnm60 & Estimated Net Migration Rate 2000s, black non-hispanic male, ages 60-64 \\
\hline r0bnm65 & Estimated Net Migration Rate 2000s, black non-hispanic male, ages 65-69 \\
\hline rObnm70 & Estimated Net Migration Rate 2000s, black non-hispanic male, ages 70-74 \\
\hline rObnm75 & Estimated Net Migration Rate 2000s, black non-hispanic male, ages 75-79 \\
\hline rObnm80 & Estimated Net Migration Rate 2000s, black non-hispanic male, ages $80-84$ \\
\hline rObnm85 & Estimated Net Migration Rate 2000s, black non-hispanic male, ages $85+$ \\
\hline rObnt0 & Estimated Net Migration Rate 2000s, black non-hispanic total, ages 0-4 \\
\hline robnt5 & Estimated Net Migration Rate 2000s, black non-hispanic total, ages 5-9 \\
\hline
\end{tabular}


rObnt10 rObnt15 rObnt20 rObnt25 rObnt30 robnt35 rObnt40 robnt45 rObnt50 robnt55 rObnt60 rObnt65 rObnt70 rObnt75 rObnt80 robnt85 rOthfo rOthf5 rOthf10 rOthf15 rOthf20 rOthf25 r0thf30 rOthf35 rOthf40 r0thf45 rOthf50 rOthf55 rothf60 r0thf65 rOthf70 r0thf75 rOthf80 rOthf85 rOthm0 rOthm5 rOthm10 rOthm15 rOthm 20 rOthm 25 rOthm 30 rOthm35 rOthm40 rothm45 rOthm50
Estimated Net Migration Rate 2000s, black non-hispanic total, ages 10-14 Estimated Net Migration Rate 2000s, black non-hispanic total, ages 15-19 Estimated Net Migration Rate 2000s, black non-hispanic total, ages 20-24 Estimated Net Migration Rate 2000s, black non-hispanic total, ages 25-29 Estimated Net Migration Rate 2000s, black non-hispanic total, ages 30-34 Estimated Net Migration Rate 2000s, black non-hispanic total, ages 35-39 Estimated Net Migration Rate 2000s, black non-hispanic total, ages 40-44 Estimated Net Migration Rate 2000s, black non-hispanic total, ages 45-49 Estimated Net Migration Rate 2000s, black non-hispanic total, ages 50-54 Estimated Net Migration Rate 2000s, black non-hispanic total, ages 55-59 Estimated Net Migration Rate 2000s, black non-hispanic total, ages 60-64 Estimated Net Migration Rate 2000s, black non-hispanic total, ages 65-69 Estimated Net Migration Rate 2000s, black non-hispanic total, ages 70-74 Estimated Net Migration Rate 2000s, black non-hispanic total, ages 75-79 Estimated Net Migration Rate 2000s, black non-hispanic total, ages 80-84 Estimated Net Migration Rate 2000s, black non-hispanic total, ages 85+ Estimated Net Migration Rate 2000s, total hispanic female, ages 0-4 Estimated Net Migration Rate 2000s, total hispanic female, ages 5-9 Estimated Net Migration Rate 2000s, total hispanic female, ages 10-14 Estimated Net Migration Rate in 2000s, total hispanic female, ages 15-19 Estimated Net Migration Rate in 2000s, total hispanic female, ages 20-24 Estimated Net Migration Rate in 2000s, total hispanic female, ages 25-29 Estimated Net Migration Rate in 2000s, total hispanic female, ages 30-34 Estimated Net Migration Rate in 2000s, total hispanic female, ages 35-39 Estimated Net Migration Rate in 2000s, total hispanic female, ages 40-44 Estimated Net Migration Rate in 2000s, total hispanic female, ages 45-49 Estimated Net Migration Rate in 2000s, total hispanic female, ages 50-54 Estimated Net Migration Rate in 2000s, total hispanic female, ages 55-59 Estimated Net Migration Rate in 2000s, total hispanic female, ages 60-64 Estimated Net Migration Rate in 2000s, total hispanic female, ages 65-69 Estimated Net Migration Rate in 2000s, total hispanic female, ages 70-74 Estimated Net Migration Rate in 2000s, total hispanic female, ages 75-79 Estimated Net Migration Rate in 2000s, total hispanic female, ages 80-84 Estimated Net Migration Rate in 2000s, total hispanic female, ages 85+ Estimated Net Migration Rate in 2000s, total hispanic male, ages 0-4 Estimated Net Migration Rate in 2000s, total hispanic male, ages 5-9 Estimated Net Migration Rate in 2000s, total hispanic male, ages 10-14 Estimated Net Migration Rate in 2000s, total hispanic male, ages 15-19 Estimated Net Migration Rate in 2000s, total hispanic male, ages 20-24 Estimated Net Migration Rate in 2000s, total hispanic male, ages 25-29 Estimated Net Migration Rate in 2000s, total hispanic male, ages 30-34 Estimated Net Migration Rate in 2000s, total hispanic male, ages 35-39 Estimated Net Migration Rate in 2000s, total hispanic male, ages 40-44 Estimated Net Migration Rate in 2000s, total hispanic male, ages 45-49 Estimated Net Migration Rate in 2000s, total hispanic male, ages 50-54 
rOthm55

rOthm60

rOthm65

rOthm70

rOthm75

rOthm80

rOthm85

rOtht0

rOtht5

r0tht10

rotht15

rotht20

rotht25

rOtht30

r0tht35

r0tht40

rotht45

rotht50

rotht55

rotht60

r0tht65

rOtht70

r0tht75

rotht80

r0tht85

rownfo

rownf5

rownf10

rOwnf15

rownf20

rOwnf25

rownf30

rOwnf35

rOwnf40

rownf45

rownf50

r0wnf55

rownf60

r0wnf65

rOwnf70

rOwnf75

r0wnf80

r0wnf85

rOwnm0

rOwnm5
Estimated Net Migration Rate in 2000s, total hispanic male, ages 55-59

Estimated Net Migration Rate in 2000s, total hispanic male, ages 60-64

Estimated Net Migration Rate in 2000s, total hispanic male, ages 65-69

Estimated Net Migration Rate in 2000s, total hispanic male, ages 70-74

Estimated Net Migration Rate in 2000s, total hispanic male, ages 75-79

Estimated Net Migration Rate in 2000s, total hispanic male, ages 80-84

Estimated Net Migration Rate in 2000s, total hispanic male, ages 85+

Estimated Net Migration Rate in 2000s, total hispanic, ages 0-4

Estimated Net Migration Rate in 2000s, total hispanic, ages 5-9

Estimated Net Migration Rate in 2000s, total hispanic, ages 10-14

Estimated Net Migration Rate in 2000s, total hispanic, ages 15-19

Estimated Net Migration Rate in 2000s, total hispanic, ages 20-24

Estimated Net Migration Rate in 2000s, total hispanic, ages 25-29

Estimated Net Migration Rate in 2000s, total hispanic, ages 30-34

Estimated Net Migration Rate in 2000s, total hispanic, ages 35-39

Estimated Net Migration Rate in 2000s, total hispanic, ages 40-44

Estimated Net Migration Rate in 2000s, total hispanic, ages 45-49

Estimated Net Migration Rate in 2000s, total hispanic, ages 50-54

Estimated Net Migration Rate in 2000s, total hispanic, ages 55-59

Estimated Net Migration Rate in 2000s, total hispanic, ages 60-64

Estimated Net Migration Rate in 2000s, total hispanic, ages 65-69

Estimated Net Migration Rate in 2000s, total hispanic, ages 70-74

Estimated Net Migration Rate in 2000s, total hispanic, ages 75-79

Estimated Net Migration Rate in 2000s, total hispanic, ages 80-84

Estimated Net Migration Rate in 2000s, total hispanic, ages 85+

Estimated Net Migration Rate in 2000s, white non-hispanic female, ages 0-4

Estimated Net Migration Rate in 2000s, white non-hispanic female, ages 5-9

Estimated Net Migration Rate in 2000s, white non-hispanic female, ages 10-14

Estimated Net Migration Rate in 2000s, white non-hispanic female, ages 15-19

Estimated Net Migration Rate in 2000s, white non-hispanic female, ages 20-24

Estimated Net Migration Rate in 2000s, white non-hispanic female, ages 25-29

Estimated Net Migration Rate in 2000s, white non-hispanic female, ages 30-34

Estimated Net Migration Rate in 2000s, white non-hispanic female, ages 35-39

Estimated Net Migration Rate in 2000s, white non-hispanic female, ages 40-44

Estimated Net Migration Rate in 2000s, white non-hispanic female, ages 45-49

Estimated Net Migration Rate in 2000s, white non-hispanic female, ages 50-54

Estimated Net Migration Rate in 2000s, white non-hispanic female, ages 55-59

Estimated Net Migration Rate in 2000s, white non-hispanic female, ages 60-64

Estimated Net Migration Rate in 2000s, white non-hispanic female, ages 65-69

Estimated Net Migration Rate in 2000s, white non-hispanic female, ages 70-74

Estimated Net Migration Rate in 2000s, white non-hispanic female, ages 75-79

Estimated Net Migration Rate in 2000s, white non-hispanic female, ages 80-84

Estimated Net Migration Rate in 2000s, white non-hispanic female, ages 85+

Estimated Net Migration Rate in 2000s, white non-hispanic male, ages 0-4

Estimated Net Migration Rate in 2000s, white non-hispanic male, ages 5-9 
rOwnm10

rOwnm15

rOwnm20

rOwnm25

rOwnm30

rOwnm35

rOwnm40

rOwnm45

rOwnm50

rOwnm55

rOwnm60

rownm65

rOwnm70

rOwnm75

rOwnm80

rOwnm85

rOwnt0

rOwnt5

rOwnt10

rOwnt15

rownt20

rOwnt25

rOwnt30

rownt35

rOwnt40

rOwnt45

rOwnt50

r0wnt55

rownt60

rOwnt65

rOwnt70

rownt75

rOwnt80

r0wnt85

rOonfo

rOonf5

rOonf10

rOonf15

rOonf20

rOonf25

rOonf30

rOonf35

rOonf40

r0onf45

rOonf50
Estimated Net Migration Rate in 2000s, white non-hispanic male, ages 10-14 Estimated Net Migration Rate in 2000s, white non-hispanic male, ages 15-19 Estimated Net Migration Rate in 2000s, white non-hispanic male, ages 20-24 Estimated Net Migration Rate in 2000s, white non-hispanic male, ages 25-29 Estimated Net Migration Rate in 2000s, white non-hispanic male, ages 30-34 Estimated Net Migration Rate in 2000s, white non-hispanic male, ages 35-39 Estimated Net Migration Rate in 2000s, white non-hispanic male, ages 40-44 Estimated Net Migration Rate in 2000s, white non-hispanic male, ages 45-49 Estimated Net Migration Rate in 2000s, white non-hispanic male, ages 50-54 Estimated Net Migration Rate in 2000s, white non-hispanic male, ages 55-59 Estimated Net Migration Rate in 2000s, white non-hispanic male, ages 60-64 Estimated Net Migration Rate in 2000s, white non-hispanic male, ages 65-69 Estimated Net Migration Rate in 2000s, white non-hispanic male, ages 70-74 Estimated Net Migration Rate in 2000s, white non-hispanic male, ages 75-79 Estimated Net Migration Rate in 2000s, white non-hispanic male, ages 80-84 Estimated Net Migration Rate in 2000s, white non-hispanic male, ages 85+ Estimated Net Migration Rate in 2000s, white non-hispanic total, ages 0-4 Estimated Net Migration Rate in 2000s, white non-hispanic total, ages 5-9 Estimated Net Migration Rate in 2000s, white non-hispanic total, ages 10-14 Estimated Net Migration Rate in 2000s, white non-hispanic total, ages 15-19 Estimated Net Migration Rate in 2000s, white non-hispanic total, ages 20-24 Estimated Net Migration Rate in 2000s, white non-hispanic total, ages 25-29 Estimated Net Migration Rate in 2000s, white non-hispanic total, ages 30-34 Estimated Net Migration Rate in 2000s, white non-hispanic total, ages 35-39 Estimated Net Migration Rate in 2000s, white non-hispanic total, ages 40-44 Estimated Net Migration Rate in 2000s, white non-hispanic total, ages 45-49 Estimated Net Migration Rate in 2000s, white non-hispanic total, ages 50-54 Estimated Net Migration Rate in 2000s, white non-hispanic total, ages 55-59 Estimated Net Migration Rate in 2000s, white non-hispanic total, ages 60-64 Estimated Net Migration Rate in 2000s, white non-hispanic total, ages 65-69 Estimated Net Migration Rate in 2000s, white non-hispanic total, ages 70-74 Estimated Net Migration Rate in 2000s, white non-hispanic total, ages 75-79 Estimated Net Migration Rate in 2000s, white non-hispanic total, ages 80-84 Estimated Net Migration Rate in 2000s, white non-hispanic total, ages 85+ Estimated Net Migration Rate in 2000s, other non-hispanic female, ages 0-4 Estimated Net Migration Rate in 2000s, other non-hispanic female, ages 5-9 Estimated Net Migration Rate in 2000s, other non-hispanic female, ages 10-14 Estimated Net Migration Rate in 2000s, other non-hispanic female, ages 15-19 Estimated Net Migration Rate in 2000s, other non-hispanic female, ages 20-24 Estimated Net Migration Rate in 2000s, other non-hispanic female, ages 25-29 Estimated Net Migration Rate in 2000s, other non-hispanic female, ages 30-34 Estimated Net Migration Rate in 2000s, other non-hispanic female, ages 35-39 Estimated Net Migration Rate in 2000s, other non-hispanic female, ages 40-44 Estimated Net Migration Rate in 2000s, other non-hispanic female, ages 45-49 Estimated Net Migration Rate in 2000s, other non-hispanic female, ages 50-54 
r0onf55 rOonf60 rOonf65 rOonf70 rOonf75 rOonf80 rOonf85 rOonm0 rOonm5 rOonm10 rOonm15 rOonm20 rOonm 25 r0onm30 rOonm35 rOonm40 rOonm45 rOonm50 rOonm55 rOonm60 r0onm65 rOonm70 rOonm75 rOonm80 rOonm85 rOont0 rOont5 rOont10 rOont15 rOont20 rOont25 rOont30 rOont35 rOont40 rOont45 rOont50 rOont55 rOont60 r0ont65 rOont70 rOont75 r0ont80 rOont85 rOttfo rOttf5
Estimated Net Migration Rate in 2000s, other non-hispanic female, ages 55-59 Estimated Net Migration Rate in 2000s, other non-hispanic female, ages 60-64 Estimated Net Migration Rate in 2000s, other non-hispanic female, ages 65-69 Estimated Net Migration Rate in 2000s, other non-hispanic female, ages 70-74 Estimated Net Migration Rate in 2000s, other non-hispanic female, ages 75-79 Estimated Net Migration Rate in 2000s, other non-hispanic female, ages 80-84 Estimated Net Migration Rate in 2000s, other non-hispanic female, ages 85+ Estimated Net Migration Rate in 2000s, other non-hispanic male, ages 0-4 Estimated Net Migration Rate in 2000s, other non-hispanic male, ages 5-9 Estimated Net Migration Rate in 2000s, other non-hispanic male, ages 10-14 Estimated Net Migration Rate in 2000s, other non-hispanic male, ages 15-19 Estimated Net Migration Rate in 2000s, other non-hispanic male, ages 20-24 Estimated Net Migration Rate in 2000s, other non-hispanic male, ages 25-29 Estimated Net Migration Rate in 2000s, other non-hispanic male, ages 30-34 Estimated Net Migration Rate in 2000s, other non-hispanic male, ages 35-39 Estimated Net Migration Rate in 2000s, other non-hispanic male, ages 40-44 Estimated Net Migration Rate in 2000s, other non-hispanic male, ages 45-49 Estimated Net Migration Rate in 2000s, other non-hispanic male, ages 50-54 Estimated Net Migration Rate in 2000s, other non-hispanic male, ages 55-59 Estimated Net Migration Rate in 2000s, other non-hispanic male, ages 60-64 Estimated Net Migration Rate in 2000s, other non-hispanic male, ages 65-69 Estimated Net Migration Rate in 2000s, other non-hispanic male, ages 70-74 Estimated Net Migration Rate in 2000s, other non-hispanic male, ages 75-79 Estimated Net Migration Rate in 2000s, other non-hispanic male, ages 80-84 Estimated Net Migration Rate in 2000s, other non-hispanic male, ages 85+ Estimated Net Migration Rate in 2000s, other non-hispanic total, ages 0-4 Estimated Net Migration Rate in 2000s, other non-hispanic total, ages 5-9 Estimated Net Migration Rate in 2000s, other non-hispanic total, ages 10-14 Estimated Net Migration Rate in 2000s, other non-hispanic total, ages 15-19 Estimated Net Migration Rate in 2000s, other non-hispanic total, ages 20-24 Estimated Net Migration Rate in 2000s, other non-hispanic total, ages 25-29 Estimated Net Migration Rate in 2000s, other non-hispanic total, ages 30-34 Estimated Net Migration Rate in 2000s, other non-hispanic total, ages 35-39 Estimated Net Migration Rate in 2000s, other non-hispanic total, ages 40-44 Estimated Net Migration Rate in 2000s, other non-hispanic total, ages 45-49 Estimated Net Migration Rate in 2000s, other non-hispanic total, ages 50-54 Estimated Net Migration Rate in 2000s, other non-hispanic total, ages 55-59 Estimated Net Migration Rate in 2000s, other non-hispanic total, ages 60-64 Estimated Net Migration Rate in 2000s, other non-hispanic total, ages 65-69 Estimated Net Migration Rate in 2000s, other non-hispanic total, ages 70-74 Estimated Net Migration Rate in 2000s, other non-hispanic total, ages 75-79 Estimated Net Migration Rate in 2000s, other non-hispanic total, ages 80-84 Estimated Net Migration Rate in 2000s, other non-hispanic total, ages 85+ Estimated Net Migration Rate in 2000s, total female, ages 0-4

Estimated Net Migration Rate in 2000s, total female, ages 5-9 
rOttf10 Estimated Net Migration Rate in 2000s, total female, ages 10-14 r0ttf15 Estimated Net Migration Rate in 2000s, total female, ages 15-19 rOttf20 Estimated Net Migration Rate in 2000s, total female, ages 20-24 rOttf25 Estimated Net Migration Rate in 2000s, total female, ages 25-29 rOttf30 Estimated Net Migration Rate in 2000s, total female, ages 30-34 rOttf35 Estimated Net Migration Rate in 2000s, total female, ages 35-39 r0ttf40 Estimated Net Migration Rate in 2000s, total female, ages 40-44 rOttf45 Estimated Net Migration Rate in 2000s, total female, ages 45-49 rOttf50 Estimated Net Migration Rate in 2000s, total female, ages 50-54 r0ttf55 Estimated Net Migration Rate in 2000s, total female, ages 55-59 rOttf60 Estimated Net Migration Rate in 2000s, total female, ages 60-64 r0ttf65 Estimated Net Migration Rate in 2000s, total female, ages 65-69 r0ttf70 Estimated Net Migration Rate in 2000s, total female, ages 70-74 rOttf75 Estimated Net Migration Rate in 2000s, total female, ages 75-79 rOttf80 Estimated Net Migration Rate in 2000s, total female, ages 80-84 rOttf85 Estimated Net Migration Rate in 2000s, total female, ages 85+ rOttm0 Estimated Net Migration Rate in 2000s, total male, ages 0-4 r0ttm5 Estimated Net Migration Rate in 2000s, total male, ages 5-9 rOttm10 Estimated Net Migration Rate in 2000s, total male, ages 10-14 rOttm15 Estimated Net Migration Rate in 2000s, total male, ages 15-19 rOttm20 Estimated Net Migration Rate in 2000s, total male, ages 20-24 rOttm25 Estimated Net Migration Rate in 2000s, total male, ages 25-29 rOttm30 Estimated Net Migration Rate in 2000s, total male, ages 30-34 rOttm35 Estimated Net Migration Rate in 2000s, total male, ages 35-39 rOttm40 Estimated Net Migration Rate in 2000s, total male, ages 40-44 rOttm45 Estimated Net Migration Rate in 2000s, total male, ages 45-49 rOttm50 Estimated Net Migration Rate in 2000s, total male, ages 50-54 r0ttm55 Estimated Net Migration Rate in 2000s, total male, ages 55-59 rOttm60 Estimated Net Migration Rate in 2000s, total male, ages 60-64 rOttm65 Estimated Net Migration Rate in 2000s, total male, ages 65-69 rOttm70 Estimated Net Migration Rate in 2000s, total male, ages 70-74 rOttm75 Estimated Net Migration Rate in 2000s, total male, ages 75-79 rOttm80 Estimated Net Migration Rate in 2000s, total male, ages 80-84 r0ttm85 Estimated Net Migration Rate in 2000s, total male, ages 85+ rOttt0 Estimated Net Migration Rate in 2000s, total, ages 0-4 rOttt5 Estimated Net Migration Rate in 2000s, total, ages 5-9 rOttt10 Estimated Net Migration Rate in 2000s, total, ages 10-14 rottt15 Estimated Net Migration Rate in 2000s, total, ages 15-19 rOttt20 Estimated Net Migration Rate in 2000s, total, ages 20-24 rOttt25 Estimated Net Migration Rate in 2000s, total, ages 25-29 rOttt30 Estimated Net Migration Rate in 2000s, total, ages 30-34 r0ttt35 Estimated Net Migration Rate in 2000s, total, ages 35-39 rOttt40 Estimated Net Migration Rate in 2000s, total, ages 40-44 rOttt45 Estimated Net Migration Rate in 2000s, total, ages 45-49 rOttt50 Estimated Net Migration Rate in 2000s, total, ages 50-54 
r0ttt55 Estimated Net Migration Rate in 2000s, total, ages 55-59

rOttt60 Estimated Net Migration Rate in 2000s, total, ages 60-64

r0ttt65 Estimated Net Migration Rate in 2000s, total, ages 65-69

rOttt70 Estimated Net Migration Rate in 2000s, total, ages 70-74

rOttt75 Estimated Net Migration Rate in 2000s, total, ages 75-79

rOttt80 Estimated Net Migration Rate in 2000s, total, ages 80-84

r0ttt85 Estimated Net Migration Rate in 2000s, total, ages 85+

m0bnf0 Estimated Number of Net Migrants in 2000s, black non-hispanic female, ages 0-4

m0bnf5

Estimated Number of Net Migrants in 2000s, black non-hispanic female, ages 5-9

m0bnf10

Estimated Number of Net Migrants in 2000s, black non-hispanic female, ages 10-14

m0bnf15

Estimated Number of Net Migrants in 2000s, black non-hispanic female, ages 15-19

m0bnf20

Estimated Number of Net Migrants in 2000s, black non-hispanic female, ages 20-24

m0bnf25

Estimated Number of Net Migrants in 2000s, black non-hispanic female, ages 25-29

m0bnf30

Estimated Number of Net Migrants in 2000s, black non-hispanic female, ages 30-34

m0bnf35

Estimated Number of Net Migrants in 2000s, black non-hispanic female, ages 35-39

m0bnf40

Estimated Number of Net Migrants in 2000s, black non-hispanic female, ages 40-44

m0bnf45

Estimated Number of Net Migrants in 2000s, black non-hispanic female, ages 45-49

m0bnf50

Estimated Number of Net Migrants in 2000s, black non-hispanic female, ages 50-54

m0bnf55

Estimated Number of Net Migrants in 2000s, black non-hispanic female, ages 55-59

m0bnf60

Estimated Number of Net Migrants in 2000s, black non-hispanic female, ages 60-64

m0bnf65

Estimated Number of Net Migrants in 2000s, black non-hispanic female, ages 65-69

m0bnf70

Estimated Number of Net Migrants in 2000s, black non-hispanic female, ages 70-74

m0bnf75

Estimated Number of Net Migrants in 2000s, black non-hispanic female, ages 75-79

m0bnf80

Estimated Number of Net Migrants in 2000s, black non-hispanic female, ages 80-84

m0bnf85

Estimated Number of Net Migrants in 2000s, black non-hispanic female, ages 85+

m0bnm0

Estimated Number of Net Migrants in 2000s, black non-hispanic male, ages 0-4

m0bnm5

Estimated Number of Net Migrants in 2000s, black non-hispanic male, ages 5-9

m0bnm10

Estimated Number of Net Migrants in 2000s, black non-hispanic male, ages 10-14

m0bnm15

Estimated Number of Net Migrants in 2000s, black non-hispanic male, ages 15-19

m0bnm20

Estimated Number of Net Migrants in 2000s, black non-hispanic male, ages 20-24

m0bnm 25

Estimated Number of Net Migrants in 2000s, black non-hispanic male, ages 25-29

m0bnm30

Estimated Number of Net Migrants in 2000s, black non-hispanic male, ages 30-34

m0bnm35

Estimated Number of Net Migrants in 2000s, black non-hispanic male, ages 35-39

m0bnm40

Estimated Number of Net Migrants in 2000s, black non-hispanic male, ages 40-44

m0bnm45

Estimated Number of Net Migrants in 2000s, black non-hispanic male, ages 45-49

m0bnm50

Estimated Number of Net Migrants in 2000s, black non-hispanic male, ages 50-54

m0bnm55

Estimated Number of Net Migrants in 2000s, black non-hispanic male, ages 55-59

m0bnm60

Estimated Number of Net Migrants in 2000s, black non-hispanic male, ages 60-64

m0bnm65

Estimated Number of Net Migrants in 2000s, black non-hispanic male, ages 65-69

m0bnm70

Estimated Number of Net Migrants in 2000s, black non-hispanic male, ages 70-74

m0bnm75

Estimated Number of Net Migrants in 2000s, black non-hispanic male, ages 75-79

m0bnm80

Estimated Number of Net Migrants in 2000s, black non-hispanic male, ages 80-84

m0bnm85

Estimated Number of Net Migrants in 2000s, black non-hispanic male, ages 85+

m0bnt0

Estimated Number of Net Migrants in 2000s, black non-hispanic total, ages 0-4

m0bnt5

Estimated Number of Net Migrants in 2000s, black non-hispanic total, ages 5-9 
m0bnt10 Estimated Number of Net Migrants in 2000s, black non-hispanic total, ages 10-14 m0bnt15 Estimated Number of Net Migrants in 2000s, black non-hispanic total, ages 15-19 m0bnt20 Estimated Number of Net Migrants in 2000s, black non-hispanic total, ages 20-24 m0bnt25 Estimated Number of Net Migrants in 2000s, black non-hispanic total, ages 25-29 m0bnt30 Estimated Number of Net Migrants in 2000s, black non-hispanic total, ages 30-34 m0bnt35 Estimated Number of Net Migrants in 2000s, black non-hispanic total, ages 35-39 m0bnt40 Estimated Number of Net Migrants in 2000s, black non-hispanic total, ages 40-44 m0bnt45 Estimated Number of Net Migrants in 2000s, black non-hispanic total, ages 45-49 m0bnt50 Estimated Number of Net Migrants in 2000s, black non-hispanic total, ages 50-54 m0bnt55 Estimated Number of Net Migrants in 2000s, black non-hispanic total, ages 55-59 m0bnt60 Estimated Number of Net Migrants in 2000s, black non-hispanic total, ages 60-64 m0bnt65 m0bnt70 m0bnt75 Estimated Number of Net Migrants in 2000s, black non-hispanic total, ages 65-69 Estimated Number of Net Migrants in 2000s, black non-hispanic total, ages 70-74 m0bnt80 m0bnt85 Estimated Number of Net Migrants in 2000s, black non-hispanic total, ages 75-79 mothfo Estimated Number of Net Migrants in 2000s, black non-hispanic total, ages 80-84 m0thf5 m0thf10 m0thf15 m0thf20 Estimated Number of Net Migrants in 2000s, black non-hispanic total, ages 85+ Estimated Number of Net Migrants in 2000s, total hispanic female, ages 0-4 Estimated Number of Net Migrants in 2000s, total hispanic female, ages 5-9 Estimated Number of Net Migrants in 2000s, total hispanic female, ages 10-14 Estimated Number of Net Migrants in 2000s, total hispanic female, ages 15-19 m0thf25 m0thf30 Estimated Number of Net Migrants in 2000s, total hispanic female, ages 20-24 m0thf35 Estimated Number of Net Migrants in 2000s, total hispanic female, ages 25-29 Estimated Number of Net Migrants in 2000s, total hispanic female, ages 30-34 Estimated Number of Net Migrants in 2000s, total hispanic female, ages 35-39 m0thf40 m0thf45 Estimated Number of Net Migrants in 2000s, total hispanic female, ages 40-44 m0thf50 Estimated Number of Net Migrants in 2000s, total hispanic female, ages 45-49 m0thf55 m0thf60 m0thf65 Estimated Number of Net Migrants in 2000s, total hispanic female, ages 50-54 Estimated Number of Net Migrants in 2000s, total hispanic female, ages 55-59 Estimated Number of Net Migrants in 2000s, total hispanic female, ages 60-64 m0thf70 Estimated Number of Net Migrants in 2000s, total hispanic female, ages 65-69 m0thf75 m0thf80 Estimated Number of Net Migrants in 2000s, total hispanic female, ages 70-74 m0thf85 m0thm0 m0thm5 Estimated Number of Net Migrants in 2000s, total hispanic female, ages 75-79 Estimated Number of Net Migrants in 2000s, total hispanic female, ages 80-84 Estimated Number of Net Migrants in 2000s, total hispanic female, ages 85+ m0thm10 Estimated Number of Net Migrants in 2000s, total hispanic male, ages 0-4 Estimated Number of Net Migrants in 2000s, total hispanic male, ages 5-9 m0thm15 Estimated Number of Net Migrants in 2000s, total hispanic male, ages 10-14 m0thm20 m0thm 25 Estimated Number of Net Migrants in 2000s, total hispanic male, ages 15-19 Estimated Number of Net Migrants in 2000s, total hispanic male, ages 20-24 m0thm35 Estimated Number of Net Migrants in 2000s, total hispanic male, ages 25-29 m0thm40 Estimated Number of Net Migrants in 2000s, total hispanic male, ages 30-34 Estimated Number of Net Migrants in 2000s, total hispanic male, ages 35-39 m0thm45 Estimated Number of Net Migrants in 2000s, total hispanic male, ages 45-49 m0thm50 Estimated Number of Net Migrants in 2000s, total hispanic male, ages 50-54 
m0thm55 Estimated Number of Net Migrants in 2000s, total hispanic male, ages 55-59 m0thm60 Estimated Number of Net Migrants in 2000s, total hispanic male, ages 60-64 m0thm65 Estimated Number of Net Migrants in 2000s, total hispanic male, ages 65-69 m0thm70 Estimated Number of Net Migrants in 2000s, total hispanic male, ages 70-74 m0thm75 Estimated Number of Net Migrants in 2000s, total hispanic female, ages 75-79 m0thm80 Estimated Number of Net Migrants in 2000s, total hispanic male, ages 80-84 m0thm85 Estimated Number of Net Migrants in 2000s, total hispanic male, ages 85+ m0tht0 Estimated Number of Net Migrants in 2000s, total hispanic total, ages 0-4 m0tht5 Estimated Number of Net Migrants in 2000s, total hispanic total, ages 5-9 m0tht10 Estimated Number of Net Migrants in 2000s, total hispanic total, ages 10-14 m0tht15 Estimated Number of Net Migrants in 2000s, total hispanic total, ages 15-19 m0tht20 Estimated Number of Net Migrants in 2000s, total hispanic total, ages 20-24 m0tht25 Estimated Number of Net Migrants in 2000s, total hispanic total, ages 25-29 m0tht30 Estimated Number of Net Migrants in 2000s, total hispanic total, ages 30-34 m0tht35 Estimated Number of Net Migrants in 2000s, total hispanic total, ages 35-39 m0tht40 Estimated Number of Net Migrants in 2000s, total hispanic female, ages 40-44 m0tht45 Estimated Number of Net Migrants in 2000s, total hispanic total, ages 45-49 m0tht50 Estimated Number of Net Migrants in 2000s, total hispanic total, ages 50-54 m0tht55 Estimated Number of Net Migrants in 2000s, total hispanic total, ages 55-59 m0tht60 Estimated Number of Net Migrants in 2000s, total hispanic total, ages 60-64 m0tht65 Estimated Number of Net Migrants in 2000s, total hispanic total, ages 65-69 m0tht70 m0tht75 Estimated Number of Net Migrants in 2000s, total hispanic total, ages 70-74 m0tht80 Estimated Number of Net Migrants in 2000s, total hispanic total, ages 75-79 m0tht85 Estimated Number of Net Migrants in 2000s, total hispanic total, ages 80-84 m0wnfo m0wnf5 Estimated Number of Net Migrants in 2000s, total hispanic total, ages 85+ Estimated Number of Net Migrants in 2000s, white non-hispanic female, ages 0-4 m0wnf10 Estimated Number of Net Migrants in 2000s, white non-hispanic female, ages 5-9 m0wnf15 Estimated Number of Net Migrants in 2000s, white non-hispanic female, ages 10-14 m0wnf20 Estimated Number of Net Migrants in 2000s, white non-hispanic female, ages 15-19 m0wnf25 m0wnf30 m0wnf35 m0wnf40 m0wnf45 m0wnf50 m0wnf55 Estimated Number of Net Migrants in 2000s, white non-hispanic female, ages 20-24 Estimated Number of Net Migrants in 2000s, white non-hispanic female, ages 25-29 Estimated Number of Net Migrants in 2000s, white non-hispanic female, ages 30-34 Estimated Number of Net Migrants in 2000s, white non-hispanic female, ages 35-39 Estimated Number of Net Migrants in 2000s, white non-hispanic female, ages 40-44 Estimated Number of Net Migrants in 2000s, white non-hispanic female, ages 45-49 Estimated Number of Net Migrants in 2000s, white non-hispanic female, ages 50-54 Estimated Number of Net Migrants in 2000s, white non-hispanic female, ages 55-59 m0wnf60 m0wnf65 m0wnf70 m0wnf75 m0wnf80 m0wnf85 mownm0 m0wnm5 Estimated Number of Net Migrants in 2000s, white non-hispanic female, ages 60-64 Estimated Number of Net Migrants in 2000s, white non-hispanic female, ages 65-69 Estimated Number of Net Migrants in 2000s, white non-hispanic female, ages 70-74 Estimated Number of Net Migrants in 2000s, white non-hispanic female, ages 75-79 Estimated Number of Net Migrants in 2000s, white non-hispanic female, ages 80-84 Estimated Number of Net Migrants in 2000s, white non-hispanic female, ages 85+ Estimated Number of Net Migrants in 2000s, white non-hispanic male, ages 0-4 Estimated Number of Net Migrants in 2000s, white non-hispanic male, ages 5-9 
m0wnm10 m0wnm15 m0wnm20 m0wnm25 m0wnm30 m0wnm35 m0wnm40 m0wnm45 m0wnm50 m0wnm55 m0wnm60 m0wnm65 m0wnm70 m0wnm75 m0wnm80 m0wnm85 mownt0 mownt5 m0wnt10 m0wnt15 mownt20 m0wnt25 m0wnt30 m0wnt35 mownt40 m0wnt45 m0wnt50 m0wnt55 m0wnt60 m0wnt65 m0wnt70 m0wnt75 m0wnt80 m0wnt85 m0onfo m0onf5 m0onf10 m0onf15 m0onf20 m0onf25 m0onf30 m0onf35 m0onf40 m0onf45 m0onf50
Estimated Number of Net Migrants in 2000s, white non-hispanic male, ages 10-14 Estimated Number of Net Migrants in 2000s, white non-hispanic male, ages 15-19 Estimated Number of Net Migrants in 2000s, white non-hispanic male, ages 20-24 Estimated Number of Net Migrants in 2000s, white non-hispanic male, ages 25-29 Estimated Number of Net Migrants in 2000s, white non-hispanic male, ages 30-34 Estimated Number of Net Migrants in 2000s, white non-hispanic male, ages 35-39 Estimated Number of Net Migrants in 2000s, white non-hispanic male, ages 40-44 Estimated Number of Net Migrants in 2000s, white non-hispanic male, ages 45-49 Estimated Number of Net Migrants in 2000s, white non-hispanic male, ages 50-54 Estimated Number of Net Migrants in 2000s, white non-hispanic male, ages 55-59 Estimated Number of Net Migrants in 2000s, white non-hispanic male, ages 60-64 Estimated Number of Net Migrants in 2000s, white non-hispanic male, ages 65-69 Estimated Number of Net Migrants in 2000s, white non-hispanic male, ages 70-74 Estimated Number of Net Migrants in 2000s, white non-hispanic male, ages 75-79 Estimated Number of Net Migrants in 2000s, white non-hispanic male, ages 80-84 Estimated Number of Net Migrants in 2000s, white non-hispanic male, ages $85+$ Estimated Number of Net Migrants in 2000s, white non-hispanic total, ages 0-4 Estimated Number of Net Migrants in 2000s, white non-hispanic total, ages 5-9 Estimated Number of Net Migrants in 2000s, white non-hispanic total, ages 10-14 Estimated Number of Net Migrants in 2000s, white non-hispanic total, ages 15-19 Estimated Number of Net Migrants in 2000s, white non-hispanic total, ages 20-24 Estimated Number of Net Migrants in 2000s, white non-hispanic total, ages 25-29 Estimated Number of Net Migrants in 2000s, white non-hispanic total, ages 30-34 Estimated Number of Net Migrants in 2000s, white non-hispanic total, ages 35-39 Estimated Number of Net Migrants in 2000s, white non-hispanic total, ages 40-44 Estimated Number of Net Migrants in 2000s, white non-hispanic total, ages 45-49 Estimated Number of Net Migrants in 2000s, white non-hispanic total, ages 50-54 Estimated Number of Net Migrants in 2000s, white non-hispanic total, ages 55-59 Estimated Number of Net Migrants in 2000s, white non-hispanic total, ages 60-64 Estimated Number of Net Migrants in 2000s, white non-hispanic total, ages 64-69 Estimated Number of Net Migrants in 2000s, white non-hispanic total, ages 70-74 Estimated Number of Net Migrants in 2000s, white non-hispanic total, ages 75-79 Estimated Number of Net Migrants in 2000s, white non-hispanic total, ages 80-84 Estimated Number of Net Migrants in 2000s, white non-hispanic total, ages 85+ Estimated Number of Net Migrants in 2000s, other non-hispanic female, ages 0-4 Estimated Number of Net Migrants in 2000s, other non-hispanic female, ages 5-9 Estimated Number of Net Migrants in 2000s, other non-hispanic female, ages 10-14 Estimated Number of Net Migrants in 2000s, other non-hispanic female, ages 15-19 Estimated Number of Net Migrants in 2000s, other non-hispanic female, ages 20-24 Estimated Number of Net Migrants in 2000s, other non-hispanic female, ages 25-29 Estimated Number of Net Migrants in 2000s, other non-hispanic female, ages 30-34 Estimated Number of Net Migrants in 2000s, other non-hispanic female, ages 35-39 Estimated Number of Net Migrants in 2000s, other non-hispanic female, ages 40-44 Estimated Number of Net Migrants in 2000s, other non-hispanic female, ages 45-49 Estimated Number of Net Migrants in 2000s, other non-hispanic female, ages 50-54 
m0onf55 Estimated Number of Net Migrants in 2000s, other non-hispanic female, ages 55-59 m0onf60 Estimated Number of Net Migrants in 2000s, other non-hispanic female, ages 60-64 m0onf65 Estimated Number of Net Migrants in 2000s, other non-hispanic female, ages 65-69 m0onf70 Estimated Number of Net Migrants in 2000s, other non-hispanic female, ages 70-74 m0onf75 Estimated Number of Net Migrants in 2000s, other non-hispanic female, ages 75-79 m0onf80 Estimated Number of Net Migrants in 2000s, other non-hispanic female, ages 80-84 m0onf85 Estimated Number of Net Migrants in 2000s, other non-hispanic female, ages 85+ m0onm0 Estimated Number of Net Migrants in 2000s, other non-hispanic male, ages 0-4 m0onm5 Estimated Number of Net Migrants in 2000s, other non-hispanic male, ages 5-9 m0onm10 Estimated Number of Net Migrants in 2000s, other non-hispanic male, ages 10-14 m0onm15 Estimated Number of Net Migrants in 2000s, other non-hispanic male, ages 15-19 m0onm20 Estimated Number of Net Migrants in 2000s, other non-hispanic male, ages 20-24 m0onm25 Estimated Number of Net Migrants in 2000s, other non-hispanic male, ages 25-29 m0onm30 Estimated Number of Net Migrants in 2000s, other non-hispanic male, ages 30-34 m0onm35 Estimated Number of Net Migrants in 2000s, other non-hispanic male, ages 35-39 m0onm40 Estimated Number of Net Migrants in 2000s, other non-hispanic male, ages 40-44 m0onm45 Estimated Number of Net Migrants in 2000s, other non-hispanic male, ages 45-49 m0onm50 Estimated Number of Net Migrants in 2000s, other non-hispanic male, ages 50-54 m0onm55 Estimated Number of Net Migrants in 2000s, other non-hispanic male, ages 55-59 m0onm60 Estimated Number of Net Migrants in 2000s, other non-hispanic male, ages 60-64 m0onm65 Estimated Number of Net Migrants in 2000s, other non-hispanic male, ages 65-69 m0onm70 Estimated Number of Net Migrants in 2000s, other non-hispanic male, ages 70-74 m0onm75 Estimated Number of Net Migrants in 2000s, other non-hispanic male, ages 75-79 m0onm80 Estimated Number of Net Migrants in 2000s, other non-hispanic male, ages 80-84 m0onm85 Estimated Number of Net Migrants in 2000s, other non-hispanic male, ages 85+ m0ont0 Estimated Number of Net Migrants in 2000s, other non-hispanic total, ages 0-4 m0ont5 Estimated Number of Net Migrants in 2000s, other non-hispanic total, ages 5-9 m0ont10 m0ont15 Estimated Number of Net Migrants in 2000s, other non-hispanic total, ages 10-14 Estimated Number of Net Migrants in 2000s, other non-hispanic total, ages 15-19 m0ont20 m0ont25 Estimated Number of Net Migrants in 2000s, other non-hispanic total, ages 20-24 m0ont30 m0ont35 m0ont40 m0ont45 m0ont50 m0ont55 m0ont60 m0ont65 m0ont70 m0ont75 m0ont80 m0ont85 m0ttfo Estimated Number of Net Migrants in 2000s, other non-hispanic total, ages 25-29 Estimated Number of Net Migrants in 2000s, other non-hispanic total, ages 30-34 Estimated Number of Net Migrants in 2000s, other non-hispanic total, ages 35-39 Estimated Number of Net Migrants in 2000s, other non-hispanic total, ages 40-44 Estimated Number of Net Migrants in 2000s, other non-hispanic total, ages 45-49 Estimated Number of Net Migrants in 2000s, other non-hispanic total, ages 59-54 Estimated Number of Net Migrants in 2000s, other non-hispanic total, ages 55-59 Estimated Number of Net Migrants in 2000s, other non-hispanic total, ages 60-64 Estimated Number of Net Migrants in 2000s, other non-hispanic total, ages 65-69 Estimated Number of Net Migrants in 2000s, other non-hispanic total, ages 70-74 Estimated Number of Net Migrants in 2000s, other non-hispanic total, ages 75-79 Estimated Number of Net Migrants in 2000s, other non-hispanic total, ages 80-84 Estimated Number of Net Migrants in 2000s, other non-hispanic total, ages 85+ Estimated Number of Net Migrants in 2000s, total female, ages 0-4

m0ttf5 Estimated Number of Net Migrants in 2000s, total female, ages 5-9 
m0ttf10

m0ttf15

m0ttf20

m0ttf25

m0ttf30

m0ttf35

m0ttf40

m0ttf45

m0ttf50

m0ttf55

m0ttf60

m0ttf65

m0ttf70

m0ttf75

m0ttf80

m0ttf85

m0ttm0

m0ttm5

m0ttm10

m0ttm15

m0ttm20

m0ttm25

m0ttm30

m0ttm35

m0ttm40

m0ttm45

m0ttm50

m0ttm55

m0ttm60

m0ttm65

m0ttm70

m0ttm75

m0ttm80

m0ttm85

m0ttt0

m0ttt5

m0ttt10

m0ttt15

m0ttt20

m0ttt25

m0ttt30

m0ttt35

m0ttt40

m0ttt45

m0ttt50
Estimated Number of Net Migrants in 2000s, total female, ages 10-14

Estimated Number of Net Migrants in 2000s, total female, ages 15-19

Estimated Number of Net Migrants in 2000s, total female, ages 20-24

Estimated Number of Net Migrants in 2000s, total female, ages 25-29

Estimated Number of Net Migrants in 2000s, total female, ages 30-34

Estimated Number of Net Migrants in 2000s, total female, ages 35-39

Estimated Number of Net Migrants in 2000s, total female, ages 40-44

Estimated Number of Net Migrants in 2000s, total female, ages 45-49

Estimated Number of Net Migrants in 2000s, total female, ages 50-54

Estimated Number of Net Migrants in 2000s, total female, ages 55-59

Estimated Number of Net Migrants in 2000s, total female, ages 60-64

Estimated Number of Net Migrants in 2000s, total female, ages 65-69

Estimated Number of Net Migrants in 2000s, total female, ages 70-74

Estimated Number of Net Migrants in 2000s, total female, ages 75-79

Estimated Number of Net Migrants in 2000s, total female, ages 80-84

Estimated Number of Net Migrants in 2000s, total female, ages 85+

Estimated Number of Net Migrants in 2000s, total male, ages 0-4

Estimated Number of Net Migrants in 2000s, total male, ages 5-9

Estimated Number of Net Migrants in 2000s, total male, ages 10-14

Estimated Number of Net Migrants in 2000s, total male, ages 15-19

Estimated Number of Net Migrants in 2000s, total male, ages 20-24

Estimated Number of Net Migrants in 2000s, total male, ages 25-29

Estimated Number of Net Migrants in 2000s, total male, ages 30-34

Estimated Number of Net Migrants in 2000s, total male, ages 35-39

Estimated Number of Net Migrants in 2000s, total male, ages 40-44

Estimated Number of Net Migrants in 2000s, total male, ages 45-49

Estimated Number of Net Migrants in 2000s, total male, ages 50-54

Estimated Number of Net Migrants in 2000s, total male, ages 55-59

Estimated Number of Net Migrants in 2000s, total male, ages 60-64

Estimated Number of Net Migrants in 2000s, total male, ages 65-69

Estimated Number of Net Migrants in 2000s, total male, ages 70-74

Estimated Number of Net Migrants in 2000s, total male, ages 75-79

Estimated Number of Net Migrants in 2000s, total male, ages 80-84

Estimated Number of Net Migrants in 2000s, total male, ages $85+$

Estimated Number of Net Migrants in 2000s, total, ages 0-4

Estimated Number of Net Migrants in 2000s, total, ages 5-9

Estimated Number of Net Migrants in 2000s, total, ages 10-14

Estimated Number of Net Migrants in 2000s, total, ages 15-19

Estimated Number of Net Migrants in 2000s, total, ages 20-24

Estimated Number of Net Migrants in 2000s, total, ages 25-29

Estimated Number of Net Migrants in 2000s, total, ages 30-34

Estimated Number of Net Migrants in 2000s, total, ages 35-39

Estimated Number of Net Migrants in 2000s, total, ages 40-44

Estimated Number of Net Migrants in 2000s, total, ages 45-49

Estimated Number of Net Migrants in 2000s, total, ages 50-54 
m0ttt55

m0ttt60

m0ttt65

m0ttt70

m0ttt75

m0ttt80

m0ttt85

eObnf0

e0bnf5

e0bnf10

e0bnf15

e0bnf20

e0bnf25

e0bnf30

e0bnf35

e0bnf40

e0bnf45

e0bnf50

e0bnf55

e0bnf60

e0bnf65

e0bnf70

e0bnf75

e0bnf80

e0bnf85

e0bnmo

e0bnm5

e0bnm10

e0bnm15

e0bnm 20

e0bnm 25

e0bnm30

e0bnm35

eObnm40

e0bnm45

e0bnm50

e0bnm55

e0bnm60

e0bnm65

e0bnm70

e0bnm75

e0bnm80

e0bnm85

e0thfo

e0thf5
Estimated Number of Net Migrants in 2000s, total, ages 55-59

Estimated Number of Net Migrants in 2000s, total, ages 60-64

Estimated Number of Net Migrants in 2000s, total, ages 65-69

Estimated Number of Net Migrants in 2000s, total, ages 70-74

Estimated Number of Net Migrants in 2000s, total, ages 75-79

Estimated Number of Net Migrants in 2000s, total, ages 80-84

Estimated Number of Net Migrants in 2000s, total, ages 85+

Expected population count in 2010, black non-hispanic female, ages 0-4

Expected population count in 2010, black non-hispanic female, ages 5-9

Expected population count in 2010, black non-hispanic female, ages 10-14

Expected population count in 2010, black non-hispanic female, ages 15-19

Expected population count in 2010, black non-hispanic female, ages 20-24

Expected population count in 2010, black non-hispanic female, ages 25-29

Expected population count in 2010, black non-hispanic female, ages 30-34

Expected population count in 2010, black non-hispanic female, ages 35-39

Expected population count in 2010, black non-hispanic female, ages 40-44

Expected population count in 2010, black non-hispanic female, ages 45-49

Expected population count in 2010, black non-hispanic female, ages 50-54

Expected population count in 2010, black non-hispanic female, ages 55-59

Expected population count in 2010, black non-hispanic female, ages 60-64

Expected population count in 2010, black non-hispanic female, ages 65-69

Expected population count in 2010, black non-hispanic female, ages 70-74

Expected population count in 2010, black non-hispanic female, ages 75-79

Expected population count in 2010, black non-hispanic female, ages 80-84

Expected population count in 2010, black non-hispanic female, ages 85+

Expected population count in 2010, black non-hispanic male, ages 0-4

Expected population count in 2010, black non-hispanic male, ages 5-9

Expected population count in 2010, black non-hispanic male, ages 10-14

Expected population count in 2010, black non-hispanic male, ages 15-19

Expected population count in 2010, black non-hispanic male, ages 20-24

Expected population count in 2010, black non-hispanic male, ages 25-29

Expected population count in 2010, black non-hispanic male, ages 30-34

Expected population count in 2010, black non-hispanic male, ages 35-39

Expected population count in 2010, black non-hispanic male, ages 40-44

Expected population count in 2010, black non-hispanic male, ages 45-49

Expected population count in 2010, black non-hispanic male, ages 50-54

Expected population count in 2010, black non-hispanic male, ages 55-59

Expected population count in 2010, black non-hispanic male, ages 60-64

Expected population count in 2010, black non-hispanic male, ages 65-69

Expected population count in 2010, black non-hispanic male, ages 70-74

Expected population count in 2010, black non-hispanic male, ages 75-79

Expected population count in 2010, black non-hispanic male, ages 80-84

Expected population count in 2010, black non-hispanic male, ages 85+

Expected population count in 2010, total hispanic female, ages 0-4

Expected population count in 2010, total hispanic female, ages 5-9 
e0thf10 Expected population count in 2010, total hispanic female, ages 10-14 e0thf15 Expected population count in 2010, total hispanic female, ages 15-19 e0thf20 Expected population count in 2010, total hispanic female, ages 20-24 e0thf25 Expected population count in 2010, total hispanic female, ages 25-29 e0thf30 Expected population count in 2010, total hispanic female, ages 30-34 e0thf35 Expected population count in 2010, total hispanic female, ages e0thf40 Expected population count in 2010, total hispanic female, ages 40-44 e0thf45 Expected population count in 2010, total hispanic female, ages 45-49 e0thf50 Expected population count in 2010, total hispanic female, ages 50-54 e0thf55 Expected population count in 2010, total hispanic female, ages 55-59 e0thf60 Expected population count in 2010, total hispanic female, ages 60-64 e0thf65 e0thf70 e0thf75 e0thf80 e0thf85 e0thm0 e0thm5 e0thm10 e0thm15 e0thm20 e0thm 25 e0thm 30 e0thm35 e0thm40 e0thm45 e0thm50 e0thm55 e0thm60 e0thm65 e0thm70 e0thm75 e0thm80 e0thm85 Expected population count in 2010, total hispanic female, ages 65-69 Expected population count in 2010, total hispanic female, ages 70-74 Expected population count in 2010, total hispanic female, ages 75-79 Expected population count in 2010, total hispanic female, ages $80-84$ Expected population count in 2010, total hispanic female, ages $85+$ Expected population count in 2010, total hispanic male, ages 0-4 Expected population count in 2010, total hispanic male, ages 5-9 Expected population count in 2010, total hispanic male, ages 10-14 Expected population count in 2010, total hispanic male, ages 15-19 Expected population count in 2010, total hispanic male, ages 20-24 Expected population count in 2010, total hispanic male, ages 25-29 Expected population count in 2010, total hispanic male, ages 30-34 Expected population count in 2010, total hispanic male, ages 35-39 Expected population count in 2010, total hispanic male, ages 40-44 Expected population count in 2010, total hispanic male, ages 45-49 Expected population count in 2010, total hispanic male, ages 50-54 Expected population count in 2010, total hispanic male, ages 55-59 Expected population count in 2010, total hispanic male, ages 60-64 Expected population count in 2010, total hispanic male, ages 65-69 Expected population count in 2010, total hispanic male, ages 70-74 Expected population count in 2010, total hispanic male, ages 75-79 Expected population count in 2010, total hispanic male, ages 80-84 e0wnf0 Expected population count in 2010, total hispanic male, ages $85+$ e0wnf5 Expected population count in 2010, total non-hispanic male, ages 0-4 e0wnf10 Expected population count in 2010, white non-hispanic female, ages 5-9 e0wnf15 e0wnf20 e0wnf25 Expected population count in 2010, white non-hispanic female, ages 10-14 Expected population count in 2010, white non-hispanic female, ages 15-19 Expected population count in 2010, white non-hispanic female, ages 20-24 e0wnf30 e0wnf35 e0wnf40 Expected population count in 2010, white non-hispanic female, ages 25-29 Expected population count in 2010, white non-hispanic female, ages 30-34 Expected population count in 2010, white non-hispanic female, ages 35-39 e0wnf45 e0wnf50 Expected population count in 2010, white non-hispanic female, ages 40-44 Expected population count in 2010, white non-hispanic female, ages 45-49 Expected population count in 2010, white non-hispanic female, ages 50-54 
e0wnf55

e0wnf60

e0wnf65

e0wnf70

e0wnf75

e0wnf80

e0wnf85

e0wnm0

e0wnm5

e0wnm10

e0wnm15

eOwnm20

e0wnm 25

e0wnm30

e0wnm35

e0wnm40

e0wnm45

e0wnm50

e0wnm55

e0wnm60

e0wnm65

e0wnm70

e0wnm75

e0wnm80

e0wnm85

e0onfo

e0onf5

e0onf10

e0onf15

e0onf20

e0onf25

e0onf30

e0onf35

e0onf40

e0onf45

e0onf50

e0onf55

e0onf60

e0onf65

e0onf70

e0onf75

e0onf80

e0onf85

e0onm0

e0onm5
Expected population count in 2010, white non-hispanic female, ages 55-59

Expected population count in 2010, white non-hispanic female, ages 60-64

Expected population count in 2010, white non-hispanic female, ages 65-69

Expected population count in 2010, white non-hispanic female, ages 70-74

Expected population count in 2010, white non-hispanic female, ages 75-79

Expected population count in 2010, white non-hispanic female, ages $80-84$

Expected population count in 2010, white non-hispanic female, ages $85+$

Expected population count in 2010, white non-hispanic male, ages 0-4

Expected population count in 2010, white non-hispanic male, ages 5-9

Expected population count in 2010, white non-hispanic male, ages 10-14

Expected population count in 2010, white non-hispanic male, ages 15-19

Expected population count in 2010, white non-hispanic male, ages 20-24

Expected population count in 2010, white non-hispanic male, ages 25-29

Expected population count in 2010, white non-hispanic male, ages 30-34

Expected population count in 2010, white non-hispanic male, ages 35-39

Expected population count in 2010, white non-hispanic male, ages $40-44$

Expected population count in 2010, white non-hispanic male, ages 45-49

Expected population count in 2010, white non-hispanic male, ages 50-54

Expected population count in 2010, white non-hispanic male, ages 55-59

Expected population count in 2010, white non-hispanic male, ages 60-64

Expected population count in 2010, white non-hispanic male, ages 65-69

Expected population count in 2010, white non-hispanic male, ages 70-74

Expected population count in 2010, white non-hispanic male, ages 75-79

Expected population count in 2010, white non-hispanic male, ages $80-84$

Expected population count in 2010, white non-hispanic male, ages $85+$

Expected population count in 2010, other non-hispanic female, ages 0-4

Expected population count in 2010, other non-hispanic female, ages 5-9

Expected population count in 2010, other non-hispanic female, ages 10-14

Expected population count in 2010, other non-hispanic female, ages 15-19

Expected population count in 2010, other non-hispanic female, ages 20-24

Expected population count in 2010, other non-hispanic female, ages 25-29

Expected population count in 2010, other non-hispanic female, ages 30-34

Expected population count in 2010, other non-hispanic female, ages 35-39

Expected population count in 2010, other non-hispanic female, ages 40-44

Expected population count in 2010, other non-hispanic female, ages 45-49

Expected population count in 2010, other non-hispanic female, ages 50-54

Expected population count in 2010, other non-hispanic female, ages 55-59

Expected population count in 2010, other non-hispanic female, ages 60-64

Expected population count in 2010, other non-hispanic female, ages 65-69

Expected population count in 2010, other non-hispanic female, ages 70-74

Expected population count in 2010, other non-hispanic female, ages 75-79

Expected population count in 2010, other non-hispanic female, ages $80-84$

Expected population count in 2010 , other non-hispanic female, ages $85+$

Expected population count in 2010, other non-hispanic male, ages 0-4

Expected population count in 2010, other non-hispanic male, ages 5-9 
e0onm10 Expected population count in 2010, other non-hispanic male, ages 10-14 e0onm15 Expected population count in 2010, other non-hispanic male, ages 15-19 e0onm20 Expected population count in 2010, other non-hispanic male, ages 20-24 e0onm25 Expected population count in 2010, other non-hispanic male, ages 25-29 e0onm30 Expected population count in 2010, other non-hispanic male, ages 30-34 e0onm35 Expected population count in 2010, other non-hispanic male, ages 35-39 e0onm40 Expected population count in 2010, other non-hispanic male, ages 40-44 e0onm45 Expected population count in 2010, other non-hispanic male, ages 45-49 e0onm50 Expected population count in 2010, other non-hispanic male, ages 50-54 e0onm55 Expected population count in 2010, other non-hispanic male, ages 55-59 e0onm60 Expected population count in 2010, other non-hispanic male, ages 60-64 e0onm65 Expected population count in 2010, other non-hispanic male, ages 65-69 e0onm70 Expected population count in 2010, other non-hispanic male, ages 70-74 e0onm75 Expected population count in 2010, other non-hispanic male, ages 75-79 e0onm80 Expected population count in 2010, other non-hispanic male, ages 80-84 e0onm85 Expected population count in 2010, other non-hispanic male, ages 85+ e0bnt0 Expected population count in 2010, black non-hispanic total, ages 0-4 e0bnt5 Expected population count in 2010, black non-hispanic total, ages 5-9 e0bnt10 Expected population count in 2010, black non-hispanic total, ages 10-14 e0bnt15 Expected population count in 2010, black non-hispanic total, ages 15-19 e0bnt20 Expected population count in 2010, black non-hispanic total, ages 20-24 e0bnt25 Expected population count in 2010, black non-hispanic total, ages 25-29 e0bnt30 Expected population count in 2010, black non-hispanic total, ages 30-34 e0bnt35 Expected population count in 2010, black non-hispanic total, ages 35-39 e0bnt40 Expected population count in 2010, black non-hispanic total, ages 40-44 e0bnt45 Expected population count in 2010, black non-hispanic total, ages 45-49 e0bnt50 Expected population count in 2010, black non-hispanic total, ages 50-54 e0bnt55 Expected population count in 2010, black non-hispanic total, ages 55-59 e0bnt60 e0bnt65 Expected population count in 2010, black non-hispanic total, ages 60-64 Expected population count in 2010, black non-hispanic total, ages 65-69 e0bnt70 Expected population count in 2010, black non-hispanic total, ages 70-74 e0bnt75 e0bnt80 Expected population count in 2010, black non-hispanic total, ages 75-79 e0bnt85 Expected population count in 2010, black non-hispanic total, ages 80-84 e0wnt0 e0wnt5 e0wnt10 e0wnt15 e0wnt20 e0wnt25 e0wnt30 e0wnt35 e0wnt40 e0wnt45 e0wnt50 Expected population count in 2010, black non-hispanic total, ages 85+ Expected population count in 2010, white non-hispanic total, ages 0-4 Expected population count in 2010, white non-hispanic total, ages 5-9 Expected population count in 2010, white non-hispanic total, ages 10-14 Expected population count in 2010, white non-hispanic total, ages 15-19 Expected population count in 2010, white non-hispanic total, ages 20-24 Expected population count in 2010, white non-hispanic total, ages 25-29 Expected population count in 2010, white non-hispanic total, ages 30-34 Expected population count in 2010, white non-hispanic total, ages 35-39 Expected population count in 2010, white non-hispanic total, ages 40-44 Expected population count in 2010, white non-hispanic total, ages 45-49 Expected population count in 2010, white non-hispanic total, ages 50-54 
e0wnt55

e0wnt60

e0wnt65

e0wnt70

e0wnt75

e0wnt80

e0wnt85

e0ont0

e0ont5

e0ont10

e0ont15

e0ont20

e0ont25

e0ont30

e0ont35

e0ont40

e0ont45

e0ont50

e0ont55

e0ont60

e0ont65

e0ont70

e0ont75

e0ont80

e0ont85

e0tht0

e0tht5

e0tht10

e0tht15

e0tht20

e0tht 25

e0tht30

e0tht35

e0tht40

e0tht45

e0tht50

e0tht55

e0tht60

e0tht65

e0tht70

e0tht75

e0tht80

e0tht85

e0ttfo

e0ttf5
Expected population count in 2010, white non-hispanic total, ages 55-59

Expected population count in 2010, white non-hispanic total, ages 60-64

Expected population count in 2010, white non-hispanic total, ages 65-69

Expected population count in 2010, white non-hispanic total, ages 70-74

Expected population count in 2010, white non-hispanic total, ages 75-79

Expected population count in 2010, white non-hispanic total, ages 80-84

Expected population count in 2010, white non-hispanic total, ages 85+

Expected population count in 2010, other non-hispanic total, ages 0-4

Expected population count in 2010, other non-hispanic total, ages 5-9

Expected population count in 2010, other non-hispanic total, ages 10-14

Expected population count in 2010, other non-hispanic total, ages 15-19

Expected population count in 2010, other non-hispanic total, ages 20-24

Expected population count in 2010, other non-hispanic total, ages 25-29

Expected population count in 2010, other non-hispanic total, ages 30-34

Expected population count in 2010, other non-hispanic total, ages 35-39

Expected population count in 2010, other non-hispanic total, ages 40-44

Expected population count in 2010, other non-hispanic total, ages 45-49

Expected population count in 2010, other non-hispanic total, ages 50-54

Expected population count in 2010, other non-hispanic total, ages 55-59

Expected population count in 2010, other non-hispanic total, ages 60-64

Expected population count in 2010, other non-hispanic total, ages 65-69

Expected population count in 2010, other non-hispanic total, ages 70-74

Expected population count in 2010, other non-hispanic total, ages 75-79

Expected population count in 2010, other non-hispanic total, ages 80-84

Expected population count in 2010, other non-hispanic total, ages 85+

Expected population count in 2010, total hispanic, ages 0-4

Expected population count in 2010, total hispanic, ages 5-9

Expected population count in 2010, total hispanic, ages 10-14

Expected population count in 2010, total hispanic, ages 15-19

Expected population count in 2010, total hispanic, ages 20-24

Expected population count in 2010, total hispanic, ages 25-29

Expected population count in 2010, total hispanic, ages 30-34

Expected population count in 2010, total hispanic, ages 35-39

Expected population count in 2010, total hispanic, ages 40-44

Expected population count in 2010, total hispanic, ages 45-49

Expected population count in 2010, total hispanic, ages 50-54

Expected population count in 2010, total hispanic, ages 55-59

Expected population count in 2010, total hispanic, ages 60-64

Expected population count in 2010, total hispanic, ages 65-69

Expected population count in 2010, total hispanic, ages 70-74

Expected population count in 2010, total hispanic, ages 75-79

Expected population count in 2010, total hispanic, ages 80-84

Expected population count in 2010, total hispanic, ages 85+

Expected population count in 2010, total female, ages 0-4

Expected population count in 2010, total female, ages 5-9 
e0ttf10 Expected population count in 2010, total female, ages 10-14 e0ttf15 Expected population count in 2010, total female, ages 15-19 e0ttf20 Expected population count in 2010, total female, ages 20-24 e0ttf25 Expected population count in 2010, total female, ages 25-29 e0ttf30 Expected population count in 2010, total female, ages 30-34 e0ttf35 Expected population count in 2010, total female, ages 35-39 e0ttf40 Expected population count in 2010, total female, ages 40-44 e0ttf45 Expected population count in 2010, total female, ages 45-49 e0ttf50 Expected population count in 2010, total female, ages 50-54 e0ttf55 Expected population count in 2010, total female, ages 55-59 e0ttf60 Expected population count in 2010, total female, ages 60-64 e0ttf65 Expected population count in 2010, total female, ages 65-69 e0ttf70 Expected population count in 2010, total female, ages 70-74 e0ttf75 Expected population count in 2010, total female, ages 75-79 e0ttf80 Expected population count in 2010, total female, ages 80-84 e0ttf85 Expected population count in 2010, total female, ages 85+ e0ttm0 Expected population count in 2010, total male, ages 0-4 e0ttm5 Expected population count in 2010, total male, ages 5-9 e0ttm10 Expected population count in 2010, total male, ages 10-14 e0ttm15 Expected population count in 2010, total male, ages 15-19 e0ttm20 Expected population count in 2010, total male, ages 20-24 e0ttm25 Expected population count in 2010, total male, ages 25-29 e0ttm30 Expected population count in 2010, total male, ages 30-34 e0ttm35 Expected population count in 2010, total male, ages 35-39 e0ttm40 Expected population count in 2010, total male, ages 40-44 e0ttm45 Expected population count in 2010, total male, ages 45-49 e0ttm50 Expected population count in 2010, total male, ages 50-54 e0ttm55 Expected population count in 2010, total male, ages 55-59 e0ttm60 Expected population count in 2010, total male, ages 60-64 e0ttm65 Expected population count in 2010, total male, ages 65-69 e0ttm70 Expected population count in 2010, total male, ages 70-74 e0ttm75 Expected population count in 2010, total male, ages 75-79 e0ttm80 Expected population count in 2010, total male, ages 80-84 e0ttm85 Expected population count in 2010, total male, ages 85+ e0ttt0 Expected population count in 2010, total, ages 0-4 e0ttt5 Expected population count in 2010, total, ages 5-9 e0ttt10 Expected population count in 2010, total, ages 10-14 e0ttt15 Expected population count in 2010, total, ages 15-19 e0ttt20 Expected population count in 2010, total, ages 20-24 e0ttt25 Expected population count in 2010, total, ages 25-29 e0ttt30 Expected population count in 2010, total, ages 30-34 e0ttt35 Expected population count in 2010, total, ages 35-39 e0ttt40 Expected population count in 2010, total, ages 40-44 e0ttt45 Expected population count in 2010, total, ages 45-49 e0ttt50 Expected population count in 2010, total, ages 50-54 
e0ttt55 Expected population count in 2010, total, ages 55-59

e0ttt60 Expected population count in 2010, total, ages 60-64

e0ttt65 Expected population count in 2010, total, ages 65-69

e0ttt70 Expected population count in 2010, total, ages 70-74

e0ttt75 Expected population count in 2010, total, ages 75-79

e0ttt80 Expected population count in 2010, total, ages 80-84

e0ttt85 Expected population count in 2010, total, ages 85+

fObnf0

Final population count in 2010, black non-hispanic female, ages 0-4

fObnf5

f0bnf10

Final population count in 2010, black non-hispanic female, ages 5-9

fObnf15

Final population count in 2010, black non-hispanic female, ages 10-14

fObnf20

Final population count in 2010, black non-hispanic female, ages 15-19

fObnf25

Final population count in 2010, black non-hispanic female, ages 20-24

f0bnf30

Final population count in 2010, black non-hispanic female, ages 25-29

fObnf35

Final population count in 2010, black non-hispanic female, ages 30-34

fObnf40

Final population count in 2010, black non-hispanic female, ages 35-39

f0bnf45

Final population count in 2010, black non-hispanic female, ages 40-44

f0bnf50

Final population count in 2010, black non-hispanic female, ages 45-49

f0bnf55

Final population count in 2010, black non-hispanic female, ages 50-54

f0bnf60

Final population count in 2010, black non-hispanic female, ages 55-59

f0bnf65

Final population count in 2010, black non-hispanic female, ages 60-64

f0bnf70

Final population count in 2010, black non-hispanic female, ages 65-69

f0bnf75

Final population count in 2010, black non-hispanic female, ages 70-74

fObnf80

Final population count in 2010, black non-hispanic female, ages 75-79

f0bnf85

Final population count in 2010, black non-hispanic female, ages 80-84

fObnm0

Final population count in 2010, black non-hispanic female, ages $85+$

fObnm5

Final population count in 2010, black non-hispanic male, ages 0-4

fObnm10

Final population count in 2010, black non-hispanic male, ages 5-9

fObnm15

Final population count in 2010, black non-hispanic male, ages 10-14

fObnm 20

Final population count in 2010, black non-hispanic male, ages 15-19

fObnm 25

Final population count in 2010, black non-hispanic male, ages 20-24

fObnm 30

Final population count in 2010, black non-hispanic male, ages 25-29

fObnm35

Final population count in 2010, black non-hispanic male, ages 30-34

fObnm40

Final population count in 2010, black non-hispanic male, ages 35-39

fObnm45

Final population count in 2010, black non-hispanic male, ages 40-44

fObnm50

Final population count in 2010, black non-hispanic male, ages 45-49

fObnm55

Final population count in 2010, black non-hispanic male, ages 50-54

fObnm60

Final population count in 2010, black non-hispanic male, ages 55-59

fobnm65

Final population count in 2010, black non-hispanic male, ages 60-64

fobnm70

Final population count in 2010, black non-hispanic male, ages 65-69

fObnm75

Final population count in 2010, black non-hispanic male, ages 70-74

fObnm80

Final population count in 2010, black non-hispanic male, ages 75-79

fObnm85

Final population count in 2010, black non-hispanic male, ages $80-84$

fOthfo

Final population count in 2010, black non-hispanic male, ages $85+$

fOthf5

Final population count in 2010, total hispanic female, ages 0-4

Final population count in 2010 , total hispanic female, ages $4-9$ 
f0thf10 Final population count in 2010, total hispanic female, ages 10-14 f0thf15 Final population count in 2010, total hispanic female, ages 15-19 fOthf20 Final population count in 2010, total hispanic female, ages 20-24 f0thf25 Final population count in 2010, total hispanic female, ages 25-29 f0thf30 Final population count in 2010, total hispanic female, ages 30-34 f0thf35 Final population count in 2010, total hispanic female, ages 35-39 fOthf40 Final population count in 2010, total hispanic female, ages 40-44 fOthf45 Final population count in 2010, total hispanic female, ages 45-49 f0thf50 Final population count in 2010, total hispanic female, ages 50-54 f0thf55 Final population count in 2010, total hispanic female, ages 55-59 f0thf60 Final population count in 2010, total hispanic female, ages 60-64 f0thf65 Final population count in 2010, total hispanic female, ages 65-69 f0thf70 Final population count in 2010, total hispanic female, ages 70-74 f0thf75 Final population count in 2010, total hispanic female, ages 75-79 f0thf80 Final population count in 2010, total hispanic female, ages 80-84 f0thf85 Final population count in 2010, total hispanic female, ages $85+$ fothm0 Final population count in 2010, total hispanic male, ages 0-4 fothm5 Final population count in 2010, total hispanic male, ages 5-9 fOthm10 fOthm15 Final population count in 2010, total hispanic male, ages 10-14 Final population count in 2010, total hispanic male, ages 15-19 Final population count in 2010, total hispanic male, ages 20-24 Final population count in 2010, total hispanic male, ages 25-29 fOthm35 Final population count in 2010, total hispanic male, ages 30-34 Final population count in 2010, total hispanic male, ages 35-39

fothm45 Final population count in 2010, total hispanic male, ages $40-44$ fOthm50 Final population count in 2010, total hispanic male, ages 45-49 fothm55 Final population count in 2010, total hispanic male, ages 50-54 Final population count in 2010, total hispanic male, ages 55-59 Final population count in 2010, total hispanic male, ages 60-64 Final population count in 2010, total hispanic male, ages 65-69 Final population count in 2010, total hispanic male, ages 70-74

fOthm80 Final population count in 2010, total hispanic male, ages 75-79 fOthm85 Final population count in 2010, total hispanic male, ages 80-84

fOwnf0 fOwnf5

fownf10 Final population count in 2010, total hispanic male, ages $85+$

fOwnf15 Final population count in 2010, white non-hispanic female, ages 0-4 Final population count in 2010, white non-hispanic female, ages 5-9 fOwnf20 Final population count in 2010, white non-hispanic female, ages 10-14 fOwnf25 Final population count in 2010, white non-hispanic female, ages 15-19 Final population count in 2010, white non-hispanic female, ages 20-24 fownf30 f0wnf35 Final population count in 2010, white non-hispanic female, ages 25-29 Final population count in 2010, white non-hispanic female, ages 30-34 f0wnf45 Final population count in 2010, white non-hispanic female, ages 35-39 fOwnf50 Final population count in 2010, white non-hispanic female, ages 40-44 Final population count in 2010, white non-hispanic female, ages 45-49 Final population count in 2010, white non-hispanic female, ages 50-54 
fOwnf55

fownf60

f0wnf65

fOwnf70

f0wnf75

f0wnf80

f0wnf85

fownm0

fOwnm5

fOwnm10

fownm15

fownm 20

fownm 25

fOwnm 30

fownm 35

fownm40

fownm45

fOwnm50

fownm55

f0wnm60

fownm65

fOwnm70

fownm75

f0wnm80

fownm85

fOonfo

foonf5

f0onf10

f0onf15

f0onf20

f0onf25

f0onf30

f0onf35

f0onf40

f0onf45

f0onf50

f0onf55

f0onf60

f0onf65

f0onf70

f0onf75

f0onf80

f0onf85

fOonm0

foonm5
Final population count in 2010, white non-hispanic female, ages 55-59 Final population count in 2010, white non-hispanic female, ages 60-64 Final population count in 2010, white non-hispanic female, ages 65-69 Final population count in 2010, white non-hispanic female, ages 70-74 Final population count in 2010, white non-hispanic female, ages 75-79 Final population count in 2010, white non-hispanic female, ages $80-84$ Final population count in 2010, white non-hispanic female, ages $85+$ Final population count in 2010, white non-hispanic male, ages 0-4 Final population count in 2010, white non-hispanic male, ages 5-9 Final population count in 2010, white non-hispanic male, ages 10-14 Final population count in 2010, white non-hispanic male, ages 15-19 Final population count in 2010, white non-hispanic male, ages 20-24 Final population count in 2010, white non-hispanic male, ages 25-29 Final population count in 2010, white non-hispanic male, ages 30-34 Final population count in 2010, white non-hispanic male, ages 35-39 Final population count in 2010, white non-hispanic male, ages $40-44$ Final population count in 2010, white non-hispanic male, ages 45-49 Final population count in 2010, white non-hispanic male, ages 50-54 Final population count in 2010, white non-hispanic male, ages 55-59 Final population count in 2010, white non-hispanic male, ages 60-64 Final population count in 2010, white non-hispanic male, ages 65-69 Final population count in 2010, white non-hispanic male, ages 70-74 Final population count in 2010, white non-hispanic male, ages 75-79 Final population count in 2010, white non-hispanic male, ages 80-84 Final population count in 2010 , white non-hispanic male, ages $85+$ Final population count in 2010, other non-hispanic female, ages 0-4 Final population count in 2010, other non-hispanic female, ages 5-9 Final population count in 2010, other non-hispanic female, ages 10-14 Final population count in 2010, other non-hispanic female, ages 15-19 Final population count in 2010, other non-hispanic female, ages 20-24 Final population count in 2010, other non-hispanic female, ages 25-29 Final population count in 2010, other non-hispanic female, ages 30-34 Final population count in 2010, other non-hispanic female, ages 35-39 Final population count in 2010, other non-hispanic female, ages $40-44$ Final population count in 2010, other non-hispanic female, ages 45-49 Final population count in 2010, other non-hispanic female, ages 50-54 Final population count in 2010, other non-hispanic female, ages 55-59 Final population count in 2010, other non-hispanic female, ages 60-64 Final population count in 2010, other non-hispanic female, ages 65-69 Final population count in 2010, other non-hispanic female, ages 70-74 Final population count in 2010, other non-hispanic female, ages 75-79 Final population count in 2010, other non-hispanic female, ages $80-84$ Final population count in 2010, other non-hispanic female, ages $85+$ Final population count in 2010, other non-hispanic male, ages 0-4 Final population count in 2010, other non-hispanic male, ages 5-9 
fOonm10

fOonm15

f0onm20

fOonm 25

fOonm30

fOonm35

fOonm40

f0onm45

f0onm50

fOonm55

f0onm60

f0onm65

fOonm70

fOonm75

fOonm80

fOonm 85

fobnt0

fobnt5

fobnt10

fObnt15

fobnt20

fobnt 25

fObnt30

fObnt35

fobnt40

fobnt45

fobnt50

fobnt55

f0bnt60

f0bnt65

fobnt70

f0bnt75

f0bnt80

f0bnt85

fownt0

fownt5

fownt10

fownt15

fownt20

fownt25

fownt30

f0wnt35

fOwnt40

fownt45

f0wnt50
Final population count in 2010, other non-hispanic male, ages 10-14

Final population count in 2010, other non-hispanic male, ages 15-19

Final population count in 2010, other non-hispanic male, ages 20-24

Final population count in 2010, other non-hispanic male, ages 25-29

Final population count in 2010, other non-hispanic male, ages 30-34

Final population count in 2010, other non-hispanic male, ages 35-39

Final population count in 2010, other non-hispanic male, ages 40-44

Final population count in 2010, other non-hispanic male, ages 45-49

Final population count in 2010, other non-hispanic male, ages 50-54

Final population count in 2010, other non-hispanic male, ages 55-59

Final population count in 2010, other non-hispanic male, ages 60-64

Final population count in 2010, other non-hispanic male, ages 65-69

Final population count in 2010, other non-hispanic male, ages 70-74

Final population count in 2010, other non-hispanic male, ages 75-79

Final population count in 2010, other non-hispanic male, ages $80-84$

Final population count in 2010, other non-hispanic male, ages $85+$

Final population count in 2010, black non-hispanic total, ages 0-4

Final population count in 2010, black non-hispanic total, ages 5-9

Final population count in 2010, black non-hispanic total, ages 10-14

Final population count in 2010, black non-hispanic total, ages 15-19

Final population count in 2010, black non-hispanic total, ages 20-24

Final population count in 2010, black non-hispanic total, ages 25-29

Final population count in 2010, black non-hispanic total, ages 30-34

Final population count in 2010, black non-hispanic total, ages 35-39

Final population count in 2010, black non-hispanic total, ages 40-44

Final population count in 2010, black non-hispanic total, ages 45-49

Final population count in 2010, black non-hispanic total, ages 50-54

Final population count in 2010, black non-hispanic total, ages 55-59

Final population count in 2010, black non-hispanic total, ages 60-64

Final population count in 2010, black non-hispanic total, ages 65-69

Final population count in 2010, black non-hispanic total, ages 70-74

Final population count in 2010, black non-hispanic total, ages 75-79

Final population count in 2010, black non-hispanic total, ages 80-84

Final population count in 2010, black non-hispanic total, ages $85+$

Final population count in 2010, white non-hispanic total, ages 0-4

Final population count in 2010, white non-hispanic total, ages 5-9

Final population count in 2010, white non-hispanic total, ages 10-14

Final population count in 2010, white non-hispanic total, ages 15-19

Final population count in 2010, white non-hispanic total, ages 20-24

Final population count in 2010, white non-hispanic total, ages 25-29

Final population count in 2010, white non-hispanic total, ages 30-34

Final population count in 2010, white non-hispanic total, ages 35-39

Final population count in 2010, white non-hispanic total, ages 40-44

Final population count in 2010, white non-hispanic total, ages 45-49

Final population count in 2010, white non-hispanic total, ages 50-54 
fOwnt55

fownt60

f0wnt65

f0wnt70

f0wnt75

f0wnt80

f0wnt85

fOont0

fOont5

f0ont10

f0ont15

f0ont20

f0ont25

f0ont30

f0ont35

f0ont40

f0ont45

f0ont50

f0ont55

f0ont60

f0ont65

f0ont70

f0ont75

f0ont80

f0ont85

fotht0

fotht5

fOtht10

fOtht15

f0tht20

fOtht 25

f0tht30

f0tht35

f0tht40

fotht 45

f0tht50

f0tht55

fOtht60

fotht65

f0tht70

fOtht75

f0tht80

f0tht85

fOttfo

fottf5
Final population count in 2010, white non-hispanic total, ages 55-59

Final population count in 2010, white non-hispanic total, ages 60-64

Final population count in 2010, white non-hispanic total, ages 65-69

Final population count in 2010, white non-hispanic total, ages 70-74

Final population count in 2010, white non-hispanic total, ages 75-79

Final population count in 2010, white non-hispanic total, ages $80-84$

Final population count in 2010, white non-hispanic total, ages $85+$

Final population count in 2010, other non-hispanic total, ages 0-4

Final population count in 2010, other non-hispanic total, ages 5-9

Final population count in 2010, other non-hispanic total, ages 10-14

Final population count in 2010, other non-hispanic total, ages 15-19

Final population count in 2010, other non-hispanic total, ages 20-24

Final population count in 2010, other non-hispanic total, ages 25-29

Final population count in 2010, other non-hispanic total, ages 30-34

Final population count in 2010, other non-hispanic total, ages 35-39

Final population count in 2010, other non-hispanic total, ages 40-44

Final population count in 2010, other non-hispanic total, ages 45-49

Final population count in 2010, other non-hispanic total, ages 50-54

Final population count in 2010, other non-hispanic total, ages 55-59

Final population count in 2010, other non-hispanic total, ages 60-64

Final population count in 2010, other non-hispanic total, ages 65-69

Final population count in 2010, other non-hispanic total, ages 70-74

Final population count in 2010, other non-hispanic total, ages 75-79

Final population count in 2010, other non-hispanic total, ages $80-84$

Final population count in 2010, other non-hispanic total, ages $85+$

Final population count in 2010, total hispanic, ages 0-4

Final population count in 2010, total hispanic, ages 5-9

Final population count in 2010, total hispanic, ages 10-14

Final population count in 2010, total hispanic, ages 15-19

Final population count in 2010, total hispanic, ages 20-24

Final population count in 2010, total hispanic, ages 25-29

Final population count in 2010, total hispanic, ages 30-34

Final population count in 2010, total hispanic, ages 35-39

Final population count in 2010, total hispanic, ages $40-44$

Final population count in 2010, total hispanic, ages 45-49

Final population count in 2010, total hispanic, ages 50-54

Final population count in 2010, total hispanic, ages 55-59

Final population count in 2010, total hispanic, ages 60-64

Final population count in 2010, total hispanic, ages 65-69

Final population count in 2010, total hispanic, ages 70-74

Final population count in 2010, total hispanic, ages 75-79

Final population count in 2010, total hispanic, ages $80-84$

Final population count in 2010, total hispanic, ages $85+$

Final population count in 2010, total female, ages 0-4

Final population count in 2010, total female, ages 5-9 
fOttf10 Final population count in 2010, total female, ages 10-14

f0ttf15 Final population count in 2010, total female, ages 15-19

fottf20 Final population count in 2010, total female, ages 20-24

fottf25 Final population count in 2010, total female, ages 25-29

fottf30 Final population count in 2010, total female, ages 30-34

fOttf35 Final population count in 2010, total female, ages 35-39

fottf40 Final population count in 2010, total female, ages 40-44

fottf45 Final population count in 2010, total female, ages 45-49

f0ttf50 Final population count in 2010, total female, ages 50-54

f0ttf55 Final population count in 2010, total female, ages 55-59

f0ttf60 Final population count in 2010, total female, ages 60-64

f0ttf65 Final population count in 2010, total female, ages 65-69

fOttf70 Final population count in 2010, total female, ages 70-74

fottf75 Final population count in 2010, total female, ages 75-79

fOttf80 Final population count in 2010, total female, ages 80-84

fottf85 Final population count in 2010, total female, ages $85+$

fottm0 Final population count in 2010, total male, ages 0-4

fottm5 Final population count in 2010, total male, ages 5-9

fottm10 Final population count in 2010, total male, ages 10-14

fottm15 Final population count in 2010, total male, ages 15-19

f0ttm20 Final population count in 2010, total male, ages 20-24

fOttm25 Final population count in 2010, total male, ages 25-29

fottm30 Final population count in 2010, total male, ages 30-34

f0ttm35 Final population count in 2010, total male, ages 35-39

fottm40 Final population count in 2010, total male, ages $40-44$

f0ttm45 Final population count in 2010, total male, ages 45-49

f0ttm50 Final population count in 2010, total male, ages 50-54

f0ttm55 Final population count in 2010, total male, ages 55-59

fottm60 Final population count in 2010, total male, ages 60-64

f0ttm65 Final population count in 2010, total male, ages 65-69

f0ttm70 Final population count in 2010, total male, ages 70-74

fottm75 Final population count in 2010, total male, ages 75-79

fottm80 Final population count in 2010, total male, ages 80-84

fottm85 Final population count in 2010, total male, ages $85+$

fottt0 Final population count in 2010, total, ages 0-4

fottt5 Final population count in 2010, total, ages 5-9

fottt10 Final population count in 2010, total, ages 10-14

f0ttt15 Final population count in 2010, total, ages 15-19

fOttt20 Final population count in 2010, total, ages 20-24

fottt25 Final population count in 2010, total, ages 25-29

fottt30 Final population count in 2010, total, ages 30-34

f0ttt35 Final population count in 2010, total, ages 35-39

fottt40 Final population count in 2010, total, ages 40-44

fottt45 Final population count in 2010, total, ages 45-49

fottt50 Final population count in 2010, total, ages 50-54 
fOttt55 Final population count in 2010, total, ages 55-59

f0ttt60 Final population count in 2010, total, ages 60-64

f0ttt65 Final population count in 2010, total, ages 65-69

fottt70 Final population count in 2010, total, ages 70-74

fOttt75 Final population count in 2010, total, ages 75-79

f0ttt80 Final population count in 2010, total, ages 80-84

f0ttt85 Final population count in 2010, total, ages 85+

fips2

fips_integer

p0bnf0 Starting population count in 2000, black non-hispanic female, ages 0-4

p0bnf5 Starting population count in 2000, black non-hispanic female, ages 5-9

p0bnf10 Starting population count in 2000, black non-hispanic female, ages 10-14

p0bnf15 Starting population count in 2000, black non-hispanic female, ages 15-19

p0bnf20 Starting population count in 2000, black non-hispanic female, ages 20-24

p0bnf25 Starting population count in 2000, black non-hispanic female, ages 25-29

p0bnf30 Starting population count in 2000, black non-hispanic female, ages 30-34

p0bnf35 Starting population count in 2000, black non-hispanic female, ages 35-39

p0bnf40 Starting population count in 2000, black non-hispanic female, ages 40-44

p0bnf45 Starting population count in 2000, black non-hispanic female, ages 45-49

p0bnf50 Starting population count in 2000, black non-hispanic female, ages 50-54

p0bnf55 Starting population count in 2000, black non-hispanic female, ages 55-59

p0bnf60 Starting population count in 2000, black non-hispanic female, ages 60-64

p0bnf65 Starting population count in 2000, black non-hispanic female, ages 65-69

p0bnf70 Starting population count in 2000, black non-hispanic female, ages 70-74

p0bnf75 Starting population count in 2000, black non-hispanic female, ages 75-79

p0bnf80 Starting population count in 2000, black non-hispanic female, ages $80-84$

p0bnf85 Starting population count in 2000 , black non-hispanic female, ages $85+$

p0bnm0 Starting population count in 2000, black non-hispanic male, ages 0-4

p0bnm5 Starting population count in 2000, black non-hispanic male, ages 5-9

p0bnm10 Starting population count in 2000, black non-hispanic male, ages 10-14

p0bnm15 Starting population count in 2000, black non-hispanic male, ages 15-19

p0bnm20 Starting population count in 2000, black non-hispanic male, ages 20-24

p0bnm25 Starting population count in 2000, black non-hispanic male, ages 25-29

p0bnm30 Starting population count in 2000, black non-hispanic male, ages 30-34

p0bnm35 Starting population count in 2000, black non-hispanic male, ages 35-39

p0bnm40 Starting population count in 2000, black non-hispanic male, ages $40-44$

p0bnm45 Starting population count in 2000, black non-hispanic male, ages 45-49

p0bnm50 Starting population count in 2000, black non-hispanic male, ages 50-54

p0bnm55 Starting population count in 2000, black non-hispanic male, ages 55-59

p0bnm60 Starting population count in 2000, black non-hispanic male, ages 60-64

p0bnm65 Starting population count in 2000, black non-hispanic male, ages 65-69

p0bnm70 Starting population count in 2000, black non-hispanic male, ages 70-74

p0bnm75 Starting population count in 2000, black non-hispanic male, ages 75-79

p0bnm80 Starting population count in 2000, black non-hispanic male, ages $80-84$

p0bnm85 Starting population count in 2000 , black non-hispanic male, ages $85+$ 
p0bnt0 Starting population count in 2000 , black non-hispanic total, ages 0-4 p0bnt5 Starting population count in 2000, black non-hispanic total, ages 5-9 p0bnt10 Starting population count in 2000, black non-hispanic total, ages 10-14 p0bnt15 Starting population count in 2000, black non-hispanic total, ages 15-19 p0bnt20 Starting population count in 2000, black non-hispanic total, ages 20-24 p0bnt25 Starting population count in 2000, black non-hispanic total, ages 25-29 p0bnt30 Starting population count in 2000, black non-hispanic total, ages 30-34 p0bnt35 Starting population count in 2000, black non-hispanic total, ages 34-39 p0bnt40 Starting population count in 2000, black non-hispanic total, ages $40-44$ p0bnt45 Starting population count in 2000, black non-hispanic total, ages $45-49$ p0bnt50 Starting population count in 2000, black non-hispanic total, ages 50-54 p0bnt55 p0bnt60 Starting population count in 2000, black non-hispanic total, ages 55-59 p0bnt65 Starting population count in 2000 , black non-hispanic total, ages 60-64 p0bnt70 Starting population count in 2000, black non-hispanic total, ages 65-69 p0bnt75 Starting population count in 2000 , black non-hispanic total, ages 70-74 p0bnt80 Starting population count in 2000 , black non-hispanic total, ages $75-79$ p0bnt85 Starting population count in 2000 , black non-hispanic total, ages $80-84$ p0onfo p0onf5 Starting population count in 2000 , black non-hispanic total, ages $85+$ p0onf10 Starting population count in 2000 , other non-hispanic female, ages 0-4 p0onf15 p0onf20 Starting population count in 2000 , other non-hispanic female, ages 5-9 Starting population count in 2000, other non-hispanic female, ages 10-14 p0onf25 Starting population count in 2000, other non-hispanic female, ages 15-19

p0onf30 p0onf35 Starting population count in 2000, other non-hispanic female, ages 20-24 p0onf40 Starting population count in 2000, other non-hispanic female, ages 25-29 p0onf45 p0onf50 p0onf55 Starting population count in 2000, other non-hispanic female, ages 30-34 Starting population count in 2000, other non-hispanic female, ages 35-39 p0onf60 p0onf65 p0onf70 Starting population count in 2000, other non-hispanic female, ages 40-44 Starting population count in 2000, other non-hispanic female, ages 45-49 Starting population count in 2000, other non-hispanic female, ages 50-54 Starting population count in 2000, other non-hispanic female, ages 55-59 Starting population count in 2000, other non-hispanic female, ages 60-64 Starting population count in 2000, other non-hispanic female, ages 65-69 p0onf75 Starting population count in 2000, other non-hispanic female, ages 70-74 p0onf80 p0onf85 Starting population count in 2000, other non-hispanic female, ages 75-79 p0onm0 Starting population count in 2000 , other non-hispanic female, ages $80-84$ p0onm5 p0onm10 p0onm15 Starting population count in 2000 , other non-hispanic female, ages $85+$ Starting population count in 2000 , other non-hispanic male, ages 0-4 p0onm20 p0onm 25 p0onm30 p0onm35 Starting population count in 2000 , other non-hispanic male, ages 5-9 Starting population count in 2000, other non-hispanic male, ages 10-14 Starting population count in 2000, other non-hispanic male, ages 15-19 Starting population count in 2000, other non-hispanic male, ages 20-24 Starting population count in 2000, other non-hispanic male, ages 25-29 p0onm40 Starting population count in 2000, other non-hispanic male, ages 30-34 Starting population count in 2000, other non-hispanic male, ages 35-39 Starting population count in 2000, other non-hispanic male, ages $40-44$ 
p0onm45 Starting population count in 2000, other non-hispanic male, ages 45-49 p0onm50 Starting population count in 2000, other non-hispanic male, ages 50-54 p0onm55 Starting population count in 2000, other non-hispanic male, ages 55-59 p0onm60 Starting population count in 2000, other non-hispanic male, ages 60-64 p0onm65 Starting population count in 2000, other non-hispanic male, ages 65-69 p0onm70 Starting population count in 2000, other non-hispanic male, ages 70-74 p0onm75 Starting population count in 2000, other non-hispanic male, ages 75-79 p0onm80 Starting population count in 2000 , other non-hispanic male, ages $80-84$ p0onm85 Starting population count in 2000, other non-hispanic male, ages $85+$ p0ont0 Starting population count in 2000, other non-hispanic total, ages 0-4 p0ont5 Starting population count in 2000, other non-hispanic total, ages 5-9 p0ont10 Starting population count in 2000, other non-hispanic total, ages 10-14 p0ont15 Starting population count in 2000, other non-hispanic total, ages 15-19 p0ont20 Starting population count in 2000, other non-hispanic total, ages 20-24 p0ont25 Starting population count in 2000, other non-hispanic total, ages 25-29 p0ont30 Starting population count in 2000, other non-hispanic total, ages 30-34 p0ont35 Starting population count in 2000, other non-hispanic total, ages 35-39 p0ont40 Starting population count in 2000, other non-hispanic total, ages 40-44 p0ont45 Starting population count in 2000, other non-hispanic total, ages 45-49 p0ont50 Starting population count in 2000, other non-hispanic total, ages 50-54 p0ont55 Starting population count in 2000, other non-hispanic total, ages 55-59 p0ont60 Starting population count in 2000, other non-hispanic total, ages 60-64 p0ont65 Starting population count in 2000, other non-hispanic total, ages 65-69 p0ont70 Starting population count in 2000, other non-hispanic total, ages 70-74 p0ont75 Starting population count in 2000, other non-hispanic total, ages 75-79 p0ont80 Starting population count in 2000, other non-hispanic total, ages $80-84$ p0ont85 Starting population count in 2000, other non-hispanic total, ages $85+$ p0thfo Starting population count in 2000, total hispanic female, ages 0-4 p0thf5 Starting population count in 2000, total hispanic female, ages 5-9 p0thf10 Starting population count in 2000, total hispanic female, ages 10-14 p0thf15 Starting population count in 2000, total hispanic female, ages 15-19 p0thf20 Starting population count in 2000, total hispanic female, ages 20-24 p0thf25 Starting population count in 2000, total hispanic female, ages 25-29 p0thf30 Starting population count in 2000, total hispanic female, ages 30-34 p0thf35 Starting population count in 2000, total hispanic female, ages 35-39 p0thf40 Starting population count in 2000, total hispanic female, ages 40-44 p0thf45 Starting population count in 2000, total hispanic female, ages $45-49$ p0thf50 Starting population count in 2000, total hispanic female, ages 50-54 p0thf55 Starting population count in 2000, total hispanic female, ages 55-59 p0thf60 Starting population count in 2000, total hispanic female, ages 60-64 p0thf65 Starting population count in 2000, total hispanic female, ages 65-69 p0thf70 Starting population count in 2000, total hispanic female, ages $70-74$ p0thf75 Starting population count in 2000, total hispanic female, ages 75-79 p0thf80 Starting population count in 2000, total hispanic female, ages $80-84$ p0thf85 Starting population count in 2000 , total hispanic female, ages $85+$ 
p0thm0 Starting population count in 2000 , total hispanic male, ages 0-4

p0thm5 Starting population count in 2000, total hispanic male, ages 5-9

p0thm10 Starting population count in 2000, total hispanic male, ages 10-14

p0thm15 Starting population count in 2000, total hispanic male, ages 15-19

p0thm20 Starting population count in 2000, total hispanic male, ages 20-24

p0thm25 Starting population count in 2000, total hispanic male, ages 25-29

p0thm30 Starting population count in 2000, total hispanic male, ages 30-34

p0thm35 Starting population count in 2000, total hispanic male, ages 35-39

p0thm40 Starting population count in 2000, total hispanic male, ages 40-44

p0thm45 Starting population count in 2000, total hispanic male, ages 45-49

p0thm50 Starting population count in 2000, total hispanic male, ages 50-54

p0thm55 Starting population count in 2000, total hispanic male, ages 55-59

p0thm60 Starting population count in 2000, total hispanic male, ages 60-64

p0thm65 Starting population count in 2000, total hispanic male, ages 65-69

p0thm70 Starting population count in 2000, total hispanic male, ages 70-74

p0thm75 Starting population count in 2000, total hispanic male, ages 75-79

p0thm80 Starting population count in 2000, total hispanic male, ages 80-84

p0thm85 Starting population count in 2000 , total hispanic male, ages $85+$

p0tht0 Starting population count in 2000, total hispanic, ages 0-4

p0tht5 Starting population count in 2000, total hispanic, ages 5-9

p0tht10 Starting population count in 2000, total hispanic, ages 10-14

p0tht15 Starting population count in 2000, total hispanic, ages 15-19

p0tht20 Starting population count in 2000, total hispanic, ages 20-24

p0tht25 Starting population count in 2000, total hispanic, ages 25-29

p0tht30 Starting population count in 2000, total hispanic, ages 30-34

p0tht35 Starting population count in 2000, total hispanic, ages 35-39

p0tht40 Starting population count in 2000, total hispanic, ages 40-44

p0tht45 Starting population count in 2000, total hispanic, ages 45-49

p0tht50 Starting population count in 2000, total hispanic, ages 50-54

p0tht55 Starting population count in 2000, total hispanic, ages 55-59

p0tht60 Starting population count in 2000, total hispanic, ages 60-64

p0tht65 Starting population count in 2000, total hispanic, ages 65-69

p0tht70 Starting population count in 2000, total hispanic, ages 70-74

p0tht75 Starting population count in 2000, total hispanic, ages 75-79

p0tht80 Starting population count in 2000, total hispanic, ages 80-84

p0tht85 Starting population count in 2000 , total hispanic, ages $85+$

p0ttf0 Starting population count in 2000, total female, ages 0-4

p0ttf5 Starting population count in 2000, total female, ages 5-9

p0ttf10 Starting population count in 2000, total female, ages 10-14

p0ttf15 Starting population count in 2000, total female, ages 15-19

p0ttf20 Starting population count in 2000, total female, ages 20-24

p0ttf25 Starting population count in 2000, total female, ages 25-29

p0ttf30 Starting population count in 2000, total female, ages 30-34

p0ttf35 Starting population count in 2000, total female, ages 35-39

p0ttf40 Starting population count in 2000 , total female, ages $40-44$ 
p0ttf45 Starting population count in 2000, total female, ages 45-49

p0ttf50 Starting population count in 2000, total female, ages 50-54

p0ttf55 Starting population count in 2000, total female, ages 55-59

p0ttf60 Starting population count in 2000, total female, ages 60-64

p0ttf65 Starting population count in 2000, total female, ages 65-69

p0ttf70 Starting population count in 2000, total female, ages 70-74

p0ttf75 Starting population count in 2000, total female, ages 75-79

p0ttf80 Starting population count in 2000, total female, ages 80-84

p0ttf85 Starting population count in 2000 , total female, ages $85+$

p0ttm0 Starting population count in 2000, total male, ages 0-4

p0ttm5 Starting population count in 2000, total male, ages 5-9

p0ttm10 Starting population count in 2000, total male, ages 10-14

p0ttm15 Starting population count in 2000, total male, ages 15-19

p0ttm20 Starting population count in 2000, total male, ages 20-24

p0ttm25 Starting population count in 2000, total male, ages 25-29

p0ttm30 Starting population count in 2000, total male, ages 30-34

p0ttm35 Starting population count in 2000, total male, ages 35-39

p0ttm40 Starting population count in 2000, total male, ages $40-44$

p0ttm45 Starting population count in 2000, total male, ages 45-49

p0ttm50 Starting population count in 2000, total male, ages 50-54

p0ttm55 Starting population count in 2000, total male, ages 55-59

p0ttm60 Starting population count in 2000, total male, ages 60-64

p0ttm65 Starting population count in 2000, total male, ages 65-69

p0ttm70 Starting population count in 2000, total male, ages $70-74$

p0ttm75 Starting population count in 2000, total male, ages 75-79

p0ttm80 Starting population count in 2000, total male, ages $80-84$

p0ttm85 Starting population count in 2000 , total male, ages $85+$

p0ttt0 Starting population count in 2000, total, ages 0-4

p0ttt5 Starting population count in 2000, total, ages 5-9

p0ttt10 Starting population count in 2000, total, ages 10-14

p0ttt15 Starting population count in 2000, total, ages 15-19

p0ttt20 Starting population count in 2000, total, ages 20-24

p0ttt25 Starting population count in 2000, total, ages 25-29

p0ttt30 Starting population count in 2000, total, ages 30-34

p0ttt35 Starting population count in 2000, total, ages 35-39

p0ttt40 Starting population count in 2000, total, ages $40-44$

p0ttt45 Starting population count in 2000, total, ages 45-49

p0ttt50 Starting population count in 2000, total, ages 50-54

p0ttt55 Starting population count in 2000, total, ages 55-59

p0ttt60 Starting population count in 2000, total, ages 60-64

p0ttt65 Starting population count in 2000, total, ages 65-69

p0ttt70 Starting population count in 2000, total, ages 70-74

p0ttt75 Starting population count in 2000, total, ages 75-79

p0ttt80 Starting population count in 2000, total, ages 80-84

p0ttt85 Starting population count in 2000 , total, ages $85+$ 
p0wnfo Starting population count in 2000, white non-hispanic female, ages 0-4 p0wnf5 Starting population count in 2000, white non-hispanic female, ages 5-9 p0wnf10 Starting population count in 2000, white non-hispanic female, ages 10-14 p0wnf15 Starting population count in 2000, white non-hispanic female, ages 15-19 p0wnf20 Starting population count in 2000, white non-hispanic female, ages 20-24 p0wnf25 Starting population count in 2000, white non-hispanic female, ages 25-29 p0wnf30 Starting population count in 2000, white non-hispanic female, ages 30-34 p0wnf35 Starting population count in 2000, white non-hispanic female, ages 35-39 p0wnf40 Starting population count in 2000, white non-hispanic female, ages 40-44 p0wnf45 Starting population count in 2000, white non-hispanic female, ages 45-49 p0wnf50 Starting population count in 2000, white non-hispanic female, ages 50-54 p0wnf55 p0wnf60 p0wnf65 p0wnf70 p0wnf75 p0wnf80 p0wnf85 p0wnm0 p0wnm5 p0wnm10 p0wnm15 p0wnm20 p0wnm25 p0wnm30 p0wnm35 p0wnm40 p0wnm45 p0wnm50 p0wnm55 p0wnm60 p0wnm65 p0wnm70 p0wnm75 p0wnm80 p0wnm85 p0wnt0 p0wnt5 p0wnt10 Starting population count in 2000, white non-hispanic female, ages 55-59 Starting population count in 2000, white non-hispanic female, ages 60-64 Starting population count in 2000, white non-hispanic female, ages 65-69 Starting population count in 2000, white non-hispanic female, ages 70-74 Starting population count in 2000, white non-hispanic female, ages 75-79 Starting population count in 2000 , white non-hispanic female, ages $80-84$ Starting population count in 2000 , white non-hispanic female, ages $85+$ Starting population count in 2000, white non-hispanic male, ages 0-4 Starting population count in 2000, white non-hispanic male, ages 5-9 Starting population count in 2000, white non-hispanic male, ages 10-14 Starting population count in 2000, white non-hispanic male, ages 15-19 Starting population count in 2000, white non-hispanic male, ages 20-24 Starting population count in 2000, white non-hispanic male, ages 25-29 Starting population count in 2000, white non-hispanic male, ages 30-34 Starting population count in 2000, white non-hispanic male, ages 35-39 Starting population count in 2000, white non-hispanic male, ages $40-44$ Starting population count in 2000, white non-hispanic male, ages 45-49 Starting population count in 2000, white non-hispanic male, ages 50-54 Starting population count in 2000, white non-hispanic male, ages 55-59 Starting population count in 2000, white non-hispanic male, ages 60-64 Starting population count in 2000, white non-hispanic male, ages 65-69 Starting population count in 2000, white non-hispanic male, ages 70-74 Starting population count in 2000, white non-hispanic male, ages 75-79 Starting population count in 2000, white non-hispanic male, ages $80-84$ Starting population count in 2000, white non-hispanic male, ages $85+$ Starting population count in 2000, white non-hispanic total, ages 0-4 p0wnt15 Starting population count in 2000, white non-hispanic total, ages 5-9

p0wnt20 p0wnt25 p0wnt30 p0wnt35 p0wnt40
Starting population count in 2000, white non-hispanic total, ages 10-14 Starting population count in 2000, white non-hispanic total, ages 15-19 Starting population count in 2000, white non-hispanic total, ages 20-24 Starting population count in 2000, white non-hispanic total, ages 25-29 Starting population count in 2000, white non-hispanic total, ages 30-34 Starting population count in 2000, white non-hispanic total, ages 35-39 Starting population count in 2000 , white non-hispanic total, ages $40-44$ 
p0wnt45 Starting population count in 2000, white non-hispanic total, ages 45-49

p0wnt50 Starting population count in 2000, white non-hispanic total, ages 50-54

p0wnt55 Starting population count in 2000, white non-hispanic total, ages 55-59

p0wnt60 Starting population count in 2000, white non-hispanic total, ages 60-64

p0wnt65 Starting population count in 2000, white non-hispanic total, ages 65-69

p0wnt70 Starting population count in 2000, white non-hispanic total, ages 70-74

p0wnt75 Starting population count in 2000, white non-hispanic total, ages 75-79

p0wnt80 Starting population count in 2000, white non-hispanic total, ages $80-84$

p0wnt85 Starting population count in 2000, white non-hispanic total, ages $85+$

b0bnf0 Estimated number of births between 2000-2004, black non-hispanic female

b0bnf5 Estimated number of births between 2005-2010, black non-hispanic female

b0bnm0 Estimated number of births between 2000-2004, black non-hispanic male

b0bnm5 Estimated number of births between 2005-2010, black non-hispanic male

b0bnt0 Estimated number of births between 2000-2004, black non-hispanic total

b0bnt5 Estimated number of births between 2005-2010, black non-hispanic total

b0onfo Estimated number of births between 2000-2004, other non-hispanic female

b0onf5 Estimated number of births between 2005-2010, other non-hispanic female

b0onm0 Estimated number of births between 2000-2004, other non-hispanic male

b0onm5 Estimated number of births between 2005-2010, other non-hispanic male

b0ont0 Estimated number of births between 2000-2004, other non-hispanic total

b0ont5 Estimated number of births between 2005-2010, other non-hispanic total

b0thfo Estimated number of births between 2000-2004, total hispanic female

b0thf5 Estimated number of births between 2005-2010, total hispanic female

b0thm0 Estimated number of births between 2000-2004, total hispanic male

b0thm5 Estimated number of births between 2005-2010, total hispaic male

b0tht0 Estimated number of births between 2000-2004, total hispanic

b0tht5 Estimated number of births between 2005-2010, total hispanic

b0ttfo Estimated number of births between 2000-2004, total female

b0ttf5 Estimated number of births between 2005-2010, total female

b0ttm0 Estimated number of births between 2000-2004, total male

b0ttm5 Estimated number of births between 2005-2010, total male

b0ttt0 Estimated number of births between 2000-2004, total

b0ttt5 Estimated number of births between 2005-2010, total

b0wnfo Estimated number of births between 2000-2004, white non-hispanic female

b0wnf5 Estimated number of births between 2005-2010, white non-hispanic female

b0wnm0 Estimated number of births between 2000-2004, white non-hispanic male

b0wnm5 Estimated number of births between 2005-2010, white non-hispanic male

b0wnt0 Estimated number of births between 2004-2005, white non-hispanic total

b0wnt5 Estimated number of births between 2005-2010, white non-hispanic total 
Center for Demography and Ecology University of Wisconsin

1180 Observatory Drive Rm. 4412

Madison, WI 53706-1393

U.S.A.

608/262-2182

FAX 608/262-8400

comments to: rwinkler@mtu.edu 